Aus der Klinik für Unfallchirurgie, Orthopädie und Plastische Chirurgie

(Prof. Dr. med. W. Lehmann)

der Medizinischen Fakultät der Universität Göttingen

\title{
CT-Analyse zur Erfassung morphologischer Veränderungen des Hüftgelenks im Erwachsenenalter bei 1000 Patienten
}

\author{
INAUGURAL-DISSERTATION \\ zur Erlangung des Doktorgrades \\ der Medizinischen Fakultät der \\ Georg-August-Universität zu Göttingen
}

vorgelegt von

Marc-Pascal Meier

aus

Göttingen 
Dekan:

\section{Betreuungsausschuss}

Betreuer/in

Ko-Betreuer/in:

\section{Prüfungskommission}

Referent/in

PD Dr. med. T. Hawellek

Ko-Referent/in:

Drittreferent/in:
Prof. Dr. med. W. Brück

PD Dr. med. T. Hawellek

Prof. Dr. med. J. Lotz
Prof. Dr. mult. T. Meyer

Datum der mündlichen Prüfung: 20.05.2021 
Hiermit erkläre ich, die Dissertation mit dem Titel "CT-Analyse zur Erfassung morphologischer Veränderungen des Hüftgelenks im Erwachsenenalter bei 1000 Patienten" eigenständig angefertigt und keine anderen als die von mir angegebenen Quellen und Hilfsmittel verwendet zu haben.

Göttingen, den 10.05.2021

(Unterschrift) 


\section{Inhaltsverzeichnis}

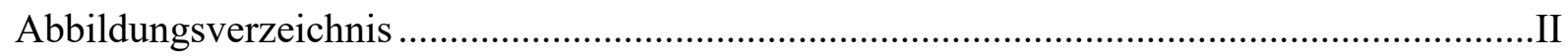

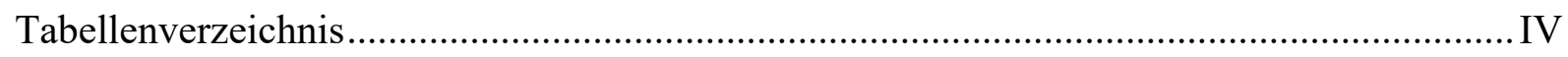

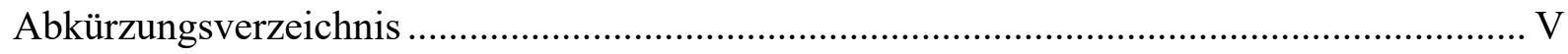

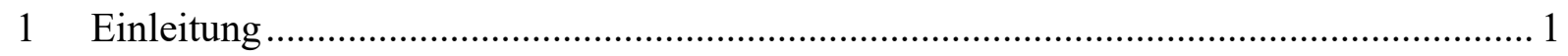

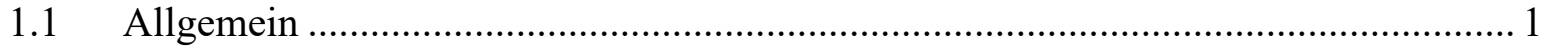

1.2 Coxarthrose

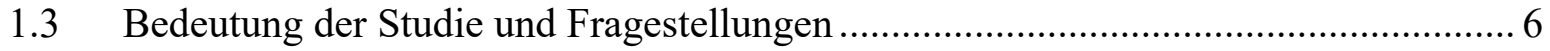

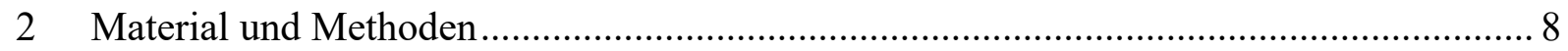

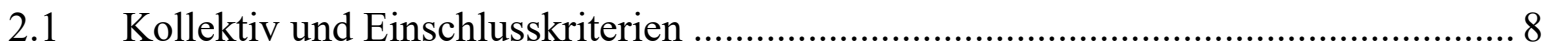

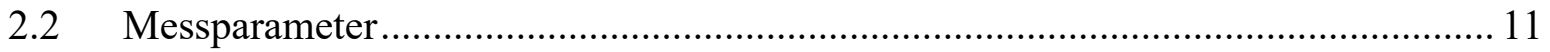

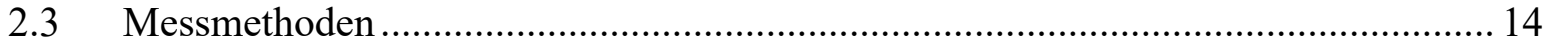

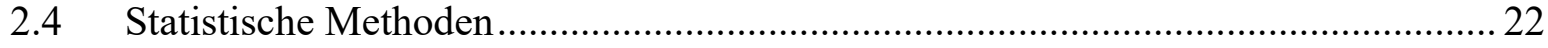

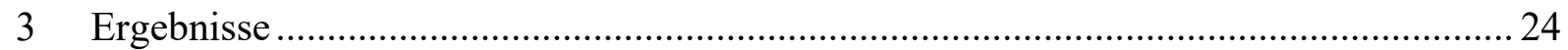

3.1 Datenverteilung der erfassten Parameter und Seitenvergleich ................................ 24

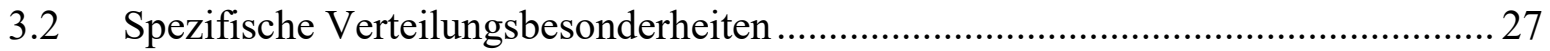

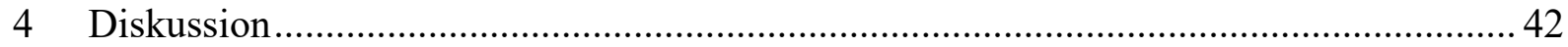

4.1 Einfluss der Seite auf die Morphologie des Hüftgelenks .......................................... 42

4.2 Einfluss des Alters auf die Morphologie des Hüftgelenks ....................................... 53

4.3 Einfluss des Geschlechts auf die Morphologie des Hüftgelenks................................ 62

4.4 Vergleich mit pathologischen Referenzwerten für die Hüftgelenkmorphologie....... 70

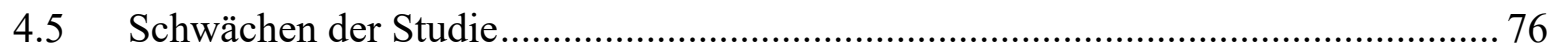

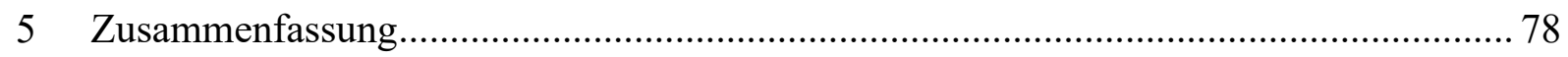

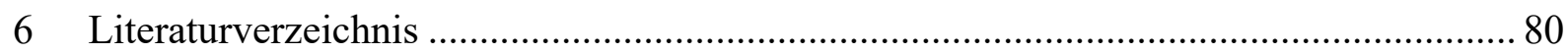




\section{Abbildungsverzeichnis}

Abb. 1 Übersicht von Ein-/Ausschlusskriterien des Studienkollektivs 9

Abb. 2 Aufteilung des Studienkollektivs nach Bildgebung und Geschlecht 10

Abb. 3 Beispielhafte Darstellung der Messungen des LCEs, CCDs und 15 SaAs

Abb. 4 Beispielhafte Darstellung der Messung des AIs 15

Abb. $5 \quad$ Arthrosegrad 4 nach Kellgren/Lawrence 16

Abb. 6 Beispielhafte Darstellung der Messung des Kopf- bzw. 16 Pfannendurchmessers

Abb. $7 \quad$ Beispielhafte Darstellung der Messung des FOs 17

Abb. $8 \quad$ Beispielhafte Darstellung der Messung des AOs 17

Abb. 9 Beispielhafte Darstellung der Messung des SWs 18

Abb. 10 Beispielhafte Darstellung der Messungen der AcetAVa, des AASAs 19 und PASAs

Abb. 11 Beispielhafte Darstellung der Messungen des AaAs und der 20 superioren Femoral neck version

Abb. 12 Beispielhafte Darstellung der Messung der inferioren Femoral neck 20 version

Abb. 13 Beispielhafte Darstellung der Messung der Beckenverkippung in 21 der Koronarebene

Abb. 14 Grafische Darstellung der Mittelwerte in Winkelgrad nach Altersgruppen unterteilt für die femoralen Messparameter

Abb. 15 Grafische Darstellung der Mittelwerte in Winkelgrad nach Altersgruppen unterteilt für die acetabulären Messparameter

Abb. 16 Grafische Darstellung der Mittelwerte nach Altersgruppen unterteilt für die Offset-Parameter sowie Femurkopf- und Acetabulumdurchmesser in $\mathrm{cm}$ und das Verhältnis F/A in \%

Abb. 17 Grafische Darstellung der Verteilung des Arthrosegrades nach Kellgren/Lawrence in den Altersgruppen G1-G4 
Abb. 18 Verteilung der physiologischen und pathologischen CCD-Werte im 37 Gesamtkollektiv nach Seite unterteilt

Abb. 19 Verteilung der physiologischen und pathologischen Femoral neck 38 version im Gesamtkollektiv nach Seite unterteilt

Abb. 20 Verteilung des physiologischen und pathologischen LCE-Winkels 39 im Gesamtkollektiv nach Seite unterteilt

Abb. 21 Verteilung des physiologischen und pathologischen AcetAVa40 Winkels im Gesamtkollektiv nach Seite unterteilt

Abb. 22 Verteilung des physiologischen und pathologischen Sharp-Winkels 41 im Gesamtkollektiv nach Seite unterteilt 


\section{Tabellenverzeichnis}

Tab. 1 Aufteilung des Studienkollektivs nach Altersgruppen 10

Tab. 2 Übersicht der erhobenen Messparameter für Acetabulum und 11 proximales Femur

Tab. 3 Klassifikation der Coxarthrose nach Kellgren/Lawrence 13

Tab. 4 Messausgleich der Parameter in der Koronarebene 22

Tab. 5 Messausgleich der Parameter in der Axialebene 22

$\begin{array}{llll}\text { Tab. } 6 \text { Mittelwerte der femoralen und acetabulären Parameter im } 24 & 4\end{array}$ Gesamtkollektiv sowie in Abhängigkeit von der Seite

Tab. 7 Verteilung der Arthrosewerte (nach Kellgren/Lawrence) im 24 Gesamtkollektiv und nach Seitenunterschieden

Tab. $8 \quad$ Verteilung der Mittelwerte für femorale und acetabuläre Parameter 27 nach Altersgruppen

Tab. 9 Verteilung der Arthrosewerte (nach Kellgren/Lawrence) nach 28 Altersgruppen

Tab. 10 Geschlechtsspezifische Verteilung der femoralen und acetabulären 33 Parameter

Tab. 11 Geschlechtsspezifische Verteilung der Arthrosewerte (nach 34 Kellgren/Lawrence)

Tab. 12 Anzahl der Hüftgelenke mit pathologischen Parametern 36 (Referenzwerte in der Literatur) 


\section{Abkürzungsverzeichnis}

AASA

AcetAVa

AI

$\mathrm{AO}$

$\mathrm{A} \alpha \mathrm{A}$

ØА

$\mathrm{BN}$

CCD

$\mathrm{CT}$

FAI

FO

FNV

F/A

ØF

G

GO

KL

LCE

Lig.

M.

MRT

PASA

SW

$\mathrm{S} \alpha \mathrm{A}$
Anteriorer acetabulärer Sektorwinkel

Acetabuläre Anteversion in der Axialebene

Acetabulärer Index

Acetabuläres Offset

Anteriorer Alpha-Winkel

Pfannendurchmesser

Bildnummer

Centrum-Collum-Diaphysen-Winkel

Computertomographie

Femoroacetabuläres Impingment

Femorales Offset

Femoral neck version

Quotient aus Femurkopfdurchmesser und Pfannendurchmesser

Femurkopfdurchmesser

Gruppe

Globales Offset

Arthrosegrad nach Kellgren/Lawrence

Lateraler-Center-Ecken-Winkel

Ligamentum

Musculus

Magnetresonanztomographie

Posteriorer acetabulärer Sektorwinkel

Sharp-Winkel

Superiorer Alpha-Winkel 


\section{$1 \quad$ Einleitung}

\subsection{Allgemein}

Das Hüftgelenk gehört mit dem Kniegelenk zu den Gelenken des Menschen, die am häufigsten von einer Arthrose betroffen sind (Dinser et al. 2013). Bis heute ist die Genese der primären Arthrose weitestgehend unverstanden (Renkawitz et al. 2011). Es wird angenommen, dass unter anderem morphologische Gelenkveränderungen Einfluss auf die Entstehung einer Arthrose haben könnten (Hasler und Hefti 2015). Beispielsweise kann eine Varus- bzw. ValgusDeformität des Kniegelenks nachweislich zur Gonarthrose führen (Harrasser et al. 2016). Eine solche Deformität wird daher auch als präarthrotische Deformität angesehen und sollte bei jungen Patienten behandelt werden, um einer frühzeitigen Arthroseentstehung entgegenzuwirken (Leunig und Ganz 2005).

Beim Hüftgelenk können morphologische Veränderungen sowohl im Bereich der acetabulären, als auch der femoralen Gelenkkomponente auftreten (Clohisy et al. 2008; van Bosse et al. 2015; Wells et al. 2017). Zur vollständigen Erfassung der Hüftgelenkmorphologie müssen für das Acetabulum und das proximale Femur einzeln und in Zusammenschau der beiden Gelenkpartner Daten im dreidimensionalen Raum erhoben werden (Gerscovich 1997). Insbesondere die vollständige dreidimensionale Erfassung des Hüftgelenks ist wichtig, um Veränderungen der artikulierenden Partner vollständig $\mathrm{zu}$ erfassen und diese unter Berücksichtigung des jeweils anderen Gelenkpartners einordnen zu können. Die Stellung des Acetabulums ist auf zweidimensionalen Standard-Röntgenaufnahmen nicht sicher zu beurteilen und auch in Bezug auf den Schenkelhals entspricht der projizierte CCD-, respektive Antetorsions-Winkel, nicht dem reellen CCD-, respektiven Antetorsions-Winkel, des Schenkelhalses auf Röntgenaufnahmen (Hasler und Hefti 2015). Um genaue Aussagen zur Morphologie des Acetabulums und proximalen Femurs treffen zu können, muss daher eine standardisierte dreidimensionale Bildgebung erfolgen. Neben der MRT- ist die CTUntersuchung hierfür geeignet. Bislang gibt es kaum Studien, die morphologische Parameter für das Hüftgelenk beim Erwachsenen dreidimensional untersucht und Standardwerte festgelegt haben.

Im Gegensatz dazu ist die Morphologie des Hüftgelenks und dessen Veränderungen im Zuge der Entwicklung vom Säugling bis in die Adoleszenz sehr genau untersucht. Normwerte und pathologische Abweichungen der Hüftgelenksmorphologie sind für diesen Altersbereich in der Literatur ausführlich beschrieben (Hasler und Hefti 2015). 
Bereits bei der U3-Vorsorgeuntersuchung des Säuglings (zumeist zwischen der vierten und sechsten postpartalen Woche) wird z. B. mittels Ultraschall nach Graf auf die morphologische Pathologie einer kongenitalen Dysplasie des Acetabulums gescreent (Graf 1984). Der Grund hierfür ist, dass die kongenitale Pfannendysplasie eine präarthrotische Deformität darstellt (Harrasser et al. 2016). Säuglinge mit einem dysplastischen Hüftgelenk werden daher je nach Ausprägungsgrad konservativ behandelt und bei konservativ therapieresistenter Dysplasie im Verlauf operiert, um der frühzeitigen Entstehung einer Coxarthrose im Erwachsenenalter entgegenzuwirken (Bernau 1990; Eberhardt et al. 2015; Fettweis 1968; Pavlik 1957; Sankar et al. 2009; Hasler und Hefti 2015; Thielemann et al. 2007).

Darüber hinaus ist auch am proximalen Femur die Veränderung der Schenkelhalsneigung in der Koronarebene im Verlauf des Wachstumsprozesses sehr genau beschrieben. Zur Erfassung wird der Centrum-Collum-Diaphysen-Winkel (CCD-Winkel) genutzt. Dieser sinkt von ca. $150^{\circ}$ (bei Geburt) auf ca. $120^{\circ}$ im Erwachsenalter (Hasler und Hefti 2015). Schenkelhalsanomalien können unbehandelt unter anderem zu Hüftdysplasien führen, in deren Folge es zur Entstehung einer Coxarthrose kommen kann (Harrasser et al. 2016). Beispielhaft können die Coxa vara $\left(\mathrm{CCD}-W i n k e l<125^{\circ}\right)$ und die Coxa valga $\left(\mathrm{CCD}\right.$-Winkel $\left.>140^{\circ}\right)$ angeführt werden (Tönnis und Heinecke 1999). Aus diesem Grund werden bei pathologischen Werten intertrochantäre Umstellungsosteotomien im Kindesalter durchgeführt (Baghdadi et al. 2018).

Neben dem CCD-Winkel kann auch die Schenkelhalsstellung in der Transversalebene als Antetorsionsstellung (Coxa antetorta/retrotorta) beschrieben werden. Milde Ausprägungsformen der Coxa antetorta/retrotorta führen zwar häufig nicht zu klinischen Beschwerden, allerdings resultieren Gangstörungen. Durch ein vermehrt innen- (Coxa antetorta) bzw. vermehrt außenrotiertes (Coxa retrotorta) Gangbild können Folgeschäden im Kniegelenk entstehen. Ausgeprägte Formen gelten als präarthrotische Deformitäten und sollten daher operativ korrigiert werden (Harrasser et al. 2016). „Ventrale Instabilität und Überlastung des Hüftgelenks können durch ausgeprägte Formen der Coxa antetorta hervorgerufen werden“ (Harrasser et al. 2016). Demgegenüber steht die Entstehung eines sogenannten Femoroacetabulären Impingement (FAI) durch die Coxa retrotorta (Harrasser et al. 2016). Hinsichtlich eines Referenzwertes besteht in der Literatur Uneinigkeit in Bezug auf die Coxa retrotorta. Beispielsweise definieren Harasser et al. (2016) diese ab einem Winkel $<10^{\circ}$, Hetsroni et al. (2013) ab einem Winkel $<5^{\circ}$. Bei der Coxa antetorta hingegen wird nahezu einheitlich ein Winkel $>25^{\circ}$ als Referenz angegeben (Harrasser et al. 2016; Hetsroni et al. 2013). 
Anhand dieser Darstellung wird deutlich, dass die morphologische Entwicklung der Hüfte bis zur Adoleszenz sehr genau untersucht wurde und klare radiologische Parameter existieren, anhand derer man sich im klinischen Alltag orientieren kann. Außerdem werden nach diesen Parametern die entsprechenden operativen Eingriffe geplant, um mittels Korrekturosteotomien einer frühzeitigen Arthroseentstehung im Hüftgelenk im weiteren Verlauf des Lebens vorzubeugen.

In der Literatur wird für das Erwachsenenalter am häufigsten das Femoroacetabuläre Impingement (FAI) als präarthrotische Deformität diskutiert. Das FAI wird in zwei Formen unterschieden, das Cam- und das Pincer-Impingement. Die Ätiologie des Cam-Impingement (Nockenwellen-Impingement) liegt in einer verminderten Taillierung des KopfSchenkelhalsübergangs. Dem Pincer-Impingement (Zangen-Impingement) hingegen liegt „eine fehlorientierte oder zu tiefe Hüftpfanne“ (vermehrte Überdachung) zu Grunde (Harrasser et al. 2016). Die häufig im Zuge eines pubertären Wachstumsschubes auftretende Epiphysiolysis capitis femoris kann ein Cam-Impingement hervorrufen (Hasler und Hefti 2015). Für beide Formen des FAIs wurden radiologische Messparameter definiert. Am proximalen Femur zeigt ein $\alpha$-Winkel $>55^{\circ}$ ein Cam-Impingement und im Bereich des Acetabulums der LCE (Lateraler-Center-Ecken-Winkel) $>40^{\circ}$ ein Pincer-Impingement an (Harrasser et al. 2016).

Saberi Hosnijeh et al. (2018) veröffentlichten kürzlich eine Longitudinalstudie, in der mittels mulitvariatem Model unterschiedliche Parameter als prädiktive Faktoren für die Entstehung einer radiologischen Coxarthrose untersucht wurden. Dabei konnte kein signifikanter Bezug zwischen dem Vorliegen eines Cam-Impingements und dem späteren Auftreten einer Coxarthrose gefunden werden (Saberi Hosnijeh et al. 2018). Diese Daten zeigen, dass aktuell weiterhin ein großer Forschungsbedarf besteht, präaarthrotische Deformitäten zu untersuchen, um deren Rolle im Arthroseentstehungsprozess besser verstehen und einordnen zu können.

Aktuell basieren Daten zur Hüftgelenkmorphologie in der adulten Lebensphase überwiegend auf Studien, die in Patienten-Kollektiven mit symptomatischem Hüftgelenk gemessen wurden (Kopec et al. 2017). Dabei bleibt letztlich unklar, ob Menschen mit asymptomatischem Hüftgelenk, die aufgrunddessen keiner radiologischen Diagnostik zugeführt wurden, nicht dennoch eine nach aktuellen Definitionen „pathologische“ radiologische Hüftgelenkmorphologie aufweisen. Außerdem weisen viele Kollektive nur kleine Fallzahlen auf ([Buller et al. 2012]: $n=115$; [Cooke et al. 2013]: $n=53$; [Hatem et al. 2017]: $n=150$ ), sodass hier nur eingeschränkt Rückschlüsse auf die Allgemeinbevölkerung gezogen werden können. 
Des Weiteren ermöglichen kleine Kollektive auch keine aussagekräftigen Vergleiche zwischen den unterschiedlichen Lebensdekaden, da die Gruppengrößen statistisch zu klein sind. Größer angelegte Studien $(n=1070)$ analysieren vergleichsweise wenige Parametern (Hartel et al. 2016), wodurch eventuelle Kompensationsmöglichkeiten in der Morphologie von Acetabulum und proximalen Femur möglicherweise unentdeckt bleiben (Widmer 2016). Darüber hinaus gibt es zahlreiche Studien, in denen die morphologischen Parameter anhand von Röntgenbildern bestimmt wurden (Nardo et al. 2015; Kopec et al. 2017). Bei diesen Studien muss davon ausgegangen werden, dass eine große Fehlermessung vorliegt, da die dreidimensionale Raumstruktur des Hüftgelenks nicht sicher durch ein zweidimensionales Röntgenbild erfasst werden kann (Cho et al. 2018; Mainard et al. 2017).

Hierdurch wird ersichtlich, dass aktuell weiterhin ein großer Forschungsbedarf besteht, radiologische Parameter zur Beschreibung der Hüftmorphologie in der erwachsenen Bevölkerung mit dreidimensionaler Bildtechnik zu untersuchen und darüber hinaus mögliche altersabhängig physiologische morphologische Veränderungen im Hüftgelenk zu identifizieren und diese klarer von pathologische Referenzparameter abgrenzen zu können.

Ein weiterer Bereich, in dem mehr Informationen über die Hüftgelenkmorphologie in Bezug auf mögliche seiten-, alters- und geschlechtsabhängige Unterschiede benötigt werden, ist die Hüftgelenkendoprothetik. Die Anzahl an neu implantierten Endoprothesen wird in den nächsten Jahren der Prognose nach deutlich ansteigen. Sowohl Studien in den USA (Bansal et al. 2014), als auch in England und Wales (Patel et al. 2015), prognostizierten eine Zunahme von 134\% an Primärimplantationen der Hüftgelenktotalendoprothesen bis zum Jahr 2030 (Patel et al 2015). Außerdem wird den Ergebnissen der Studien nach auch der prozentuale Anteil an Revisionsoperationen ansteigen. Diesbezüglich wird ein Anstieg um 31\% erwartet (Patel et al. 2015).

Diese Ausführungen verdeutlichen welche große Bedeutung die Endoprothetik inzwischen in der orthopädischen Therapie einnimmt. Für ein gutes klinisches Ergebnis ist eine optimale Therapie-/Prothesenplanung unerlässlich. Diese orientiert sich häufig an der Morphologie des geschädigten Hüftgelenks oder an der kontralateralen Seite, wenn das zu ersetzende Gelenk zu stark destruiert ist (Eggli et al. 2018; Knight and Atwater 1992; Kutzner et al. 2017).

Letztlich ist allerdings unklar, ob seitenspezifische Unterschiede bei den Hüftgelenken vorliegen und somit eine Orientierung an der kontralateralen Seite zumindest kritisch betrachtet werden sollte. 
Seiten-, alters- und geschlechtsabhängige physiologische Referenzparameter, die sowohl als Unterstützung zur Planung, als auch im Implantatdesign, Berücksichtigung finden könnten, sind bislang überwiegend nicht definiert. Die präoperative Planung der Standardprothese erfolgt zumeist anhand von zweidimensionalen Röntgenbildern (Renkawitz et al. 2011). Morphologische Referenzwerte auf Basis von dreidimensionalen Analysen könnten möglicherweise zu einer Optimierung des operativen Outcomes führen. Die Vorrausetzung hierfür ist jedoch morphologische Parameter des Hüftgelenks in einem ausreichend großen Studienkollektiv dreidimensional zu erfassen.

\subsection{Coxarthrose}

„Die Coxarthrose ist eine degenerative Erkrankung des Hüftgelenks mit unterschiedlicher Ätiologie“ (Harrasser et al. 2016; Litwic et al. 2013). Vordergründig ist eine Destruktion des Gelenkknorpels (Beck et al. 2005). Es wird in eine primäre und sekundäre Form unterschieden (Niethard et al. 2014). Die primäre Variante ist idiopathisch und macht ca. $75 \%$ der Coxarthrosen aus. Die sekundäre Form (25\%) ist durch Deformitäten, Nekrosen oder Traumata bedingt (Ackerman et al. 2017). Selten sind Arthropathien ursächlich.

Die Genese der Coxarthrose ist multifaktoriell bedingt (Murphy et al. 2016). Zum einen spielen mechanische Faktoren eine Rolle, zum anderen nicht-mechanische Faktoren. Der anfänglichen Knorpelschädigung folgt eine Destruktion, die letztlich auch zu einer knöchernen Begleitreaktion führt (Murphy et al. 2016).

Die Erkrankungsinzidenz liegt bei ca. 10-195/100.000 Personen. Typische Leitsymptome sind bei fortgeschrittener Coxarthrose Anlaufschmerzen und belastungsinduzierte Schmerzen, die zumeist in der Leiste lokalisiert sind, aber durchaus auch in den Oberschenkel ausstrahlen können. Ferner tritt auch häufig im späteren Krankheitsstadium ein Ruhe- und Nachtschmerz auf. Des Weiteren kann bei vielen Patienten eine unterschiedlich stark ausgeprägte Morgensteifigkeit auftreten (Harrasser et al. 2016). Begleiterscheinungen sind häufig muskuläre Insuffizienzen, die in einen Funktionsverlust resultieren (Bijlsma et al. 2011).

Die Diagnostik kann über klinische Tests oder über Bildgebungsmethoden unter Anwendung von Arthrose-Scores, wie z. B. der Klassifikation nach Kellgren/Lawrence erfolgen (Kellgren und Lawrence 1952; Kellgren und Lawrence 1957). Radiologische Arthrosezeichen sind eine Gelenkspaltverschmälerung, subchondrale Sklerosierung, osteophytäre Randanbauten und Geröllzysten (Altman et al. 1991). 
Differenzialdiagnostisch sollten Hernien, radikuläre Erkrankungen der Lendenwirbelsäule mit Ausstrahlungssymptomatik und funktionelle Gelenkblockaden ausgeschlossen werden (Zengini et al. 2016). Therapieoptionen können konservativer („Bewegung bei geringer Belastung“, Analgetika oder Physiotherapie u. a.) oder operativer (gelenkerhaltend oder gelenkersetzend) Natur sein (Harrasser et al. 2016).

\subsection{Bedeutung der Studie und Fragestellungen}

Wird die aktuelle Studienlage zusammenfassend betrachtet, zeigt sich, dass es bislang keine Studie gibt, in der dreidimensional die Hüftgelenkmorphologie in einem nicht symptomatischen Hüftkollektiv mit aussagekräftiger hoher Fallzahl untersucht wurde und dabei eine Erhebung verschiedenster hüftmorphologischer Parameter, sowohl der acetabulären, als auch femoralen Gelenkkomponente, erfolgte. Die aktuell in der Literatur diskutierten Referenzwerte stammen größtenteils aus Patientenkollektiven mit symptomatischen Hüftgelenken, wobei sich diese teilweise je nach Studie unterscheiden und letztlich die Frage offen bleibt, ob auch bei symptomfreien Hüftgelenken ,,pathologische“ Hüftparameter gefunden werden können.

Darüber hinaus suggeriert die aktuelle Datenlage, dass die morphologische Entwicklung des Hüftgelenks nach der Adoleszenz-Phase abgeschlossen sei und somit unabhängig vom Alter immer gleiche Referenzparameter gelten sollten. Ob dies tatsächlich so ist oder ob nicht eine physiologische Veränderung der Hüftgelenkmorphologie auch im Erwachsenenalter stattfindet, soll weiterhin Ziel dieser Studie sein.

Die Kenntnis über spezifische „adulte Referenzparameter“ sind für die Therapie und auch bei operativen Maßnahmen, wie Umstellungsosteotomien oder Totalendoprothesenimplantationen wichtig.

Unter Berücksichtigung all dieser Aspekte hat es sich die vorliegende Arbeit zum Ziel gesetzt, in einem primär asymptomatischen adulten Kollektiv mit hoher Fallzahl, eine Vielzahl von hüftmorphologischen Parametern zu messen, um eine gesamtheitliche Darstellung der hüftmorphologischen Entwicklung des Erwachsenalters, sofern diese existiert, zu erfassen.

Da die Studie von rein retrospektivem Charakter ist und keine Schmerzanamnese der Patienten, von denen die radiologischen Aufnahmen stammen, erhoben wurde, sollte die Erfassung des Arthrosegrades sicherstellen, dass tatsächlich ein überwiegend asymptomatisches Kollektiv vorliegt. 
Auf dieser Basis wurde untersucht, in welchem Referenzbereich morphologische Parameter von Acetabulum und Femur im Göttinger-Kollektiv auftreten und wie hoch deren Arthrosegrad ist (1). Ein weiteres Hauptaugenmerk lag auf Seitendifferenzen (2), Altersunterschieden (3) und geschlechtsspezifischen Unterschieden (4). Letztlich sollte ein Vergleich zwischen den gemessenen Werten des Göttinger-Kollektivs und denen als „physiologisch“/,pathologisch“ definierten Werten der Vergleichsstudien erfolgen (5). Die Ergebnisse der vorliegenden Arbeit könnten für die klinische Praxis sowohl in Bezug auf Diagnostik, als auch für die Therapie von Hüftschmerzen, von großer Bedeutung sein. 


\section{Material und Methoden}

\subsection{Kollektiv und Einschlusskriterien}

Im Rahmen dieser Arbeit wurden computertomographisch Hüftgelenke von 1000 Patienten retrospektiv analysiert. Hierzu wurden Computertomographien von Polytrauma- und AngioScans ausgewertet, bei denen Becken sowie die Femora beidseits vollständig erfasst waren. Die CT-Datensätze waren im Rahmen der Diagnostik von Patienten aus der Klinik für Thorax-, Herz- und Gefäßchirurgie sowie aus der Klinik für Unfallchirurgie, Orthopädie und Plastische Chirurgie zwischen 2007 und 2017 in der Klinik für diagnostische Radiologie der Universitätsmedizin Göttingen entstanden. Die Studie wurde von der Ethikkommission Göttingen genehmigt (Antragsnummer: 18/8/17).

Alle Patienten hatten beim Ausfüllen der Untersuchungsbögen (erfolgte vor der Bildgebung) zugestimmt, dass ihre Daten für wissenschaftliche Studien verwendet werden dürfen. Da die Studie von rein deskriptivem Charakter ist, erfolgte keine Kontaktaufnahme zu den Patienten.

Nach durchgeführter Qualitätsprüfung wurden einige Messungen exkludiert. Als Einschlusskriterium galt, dass sämtliche Messparameter bilateral bei beiden Hüftgelenken vollständig erhoben werden konnten. Darüber hinaus wurden nur Patienten ab einem Alter von 18 Jahren zum Zeitpunkt der durchgeführten CT-Untersuchung eingeschlossen. Patienten mit Hüftprothesenimplantationen, einliegenden Osteosynthesematerialien, Frakturen, Dislokationen, Luxationen oder Osteodestruktion wurden exkludiert. Insgesamt wurden 288 Messungen ausgeschlossen, sodass das Kollektiv letztlich aus 712 Patienten mit 1424 Hüftgelenken bestand (Abbildung 1). 
Initial untersuchte und vermessene Patienten $(\mathrm{n}=1000)$

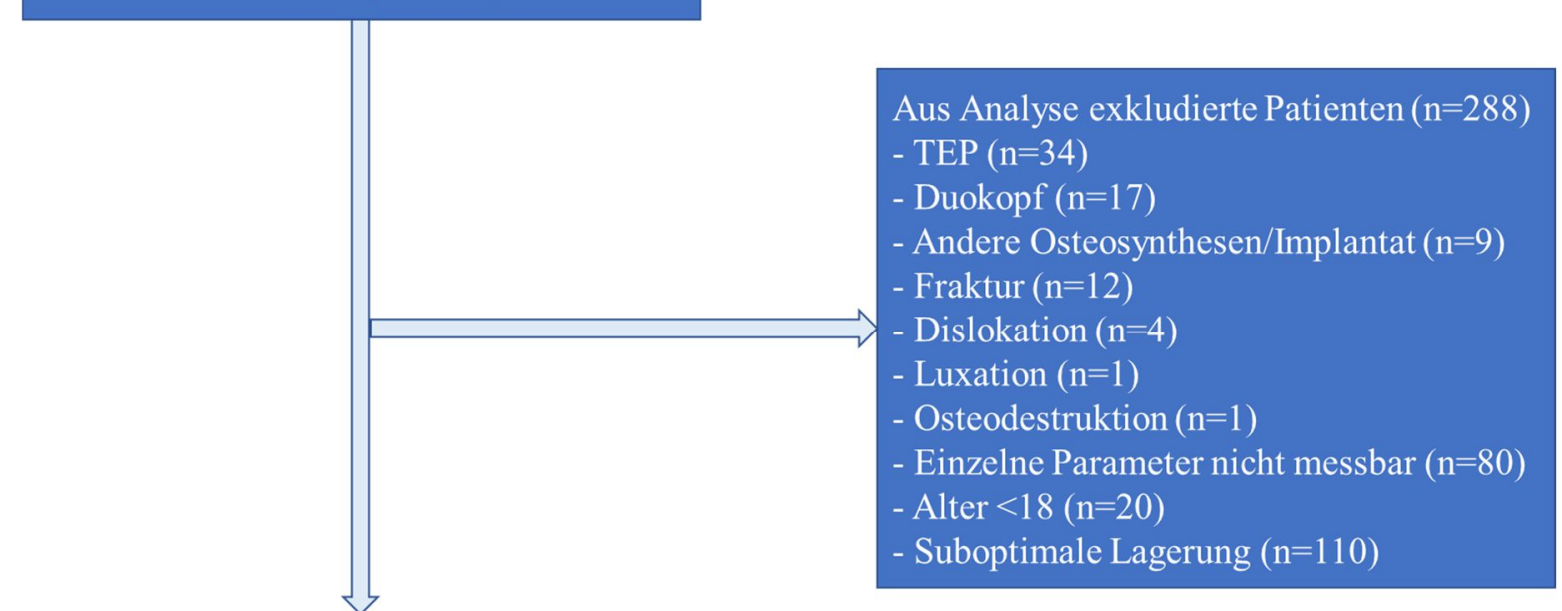

In Analyse inkludierte Patienten mit vollständiger bilateraler Messung aller

Parameter $(\mathrm{n}=712)$

Abb. 1 Übersicht von Ein-/Ausschlusskriterien des Studienkollektivs

Die 712 untersuchten Patienten hatten ein Durchschnittsalter von 59 Jahren. 66,2\% der Fälle waren männlich (471/712) und 33,8\% weiblich (241/712). Es wurden 575 CT-Angio-Scans der Kategorie Becken-/Beingefäße und 137 Polytrauma-CTs ausgewertet. 373 Messungen der Männer stammen aus CT-Angio-Scans und 98 aus CT-Polytrauma-Scans. Bei den Frauen beträgt das Verhältnis 202 (CT-Angio-Scans) zu 39 (CT-Polytrauma-Scans). Abbildung 2 zeigt eine Übersicht der Kollektivverteilung. 


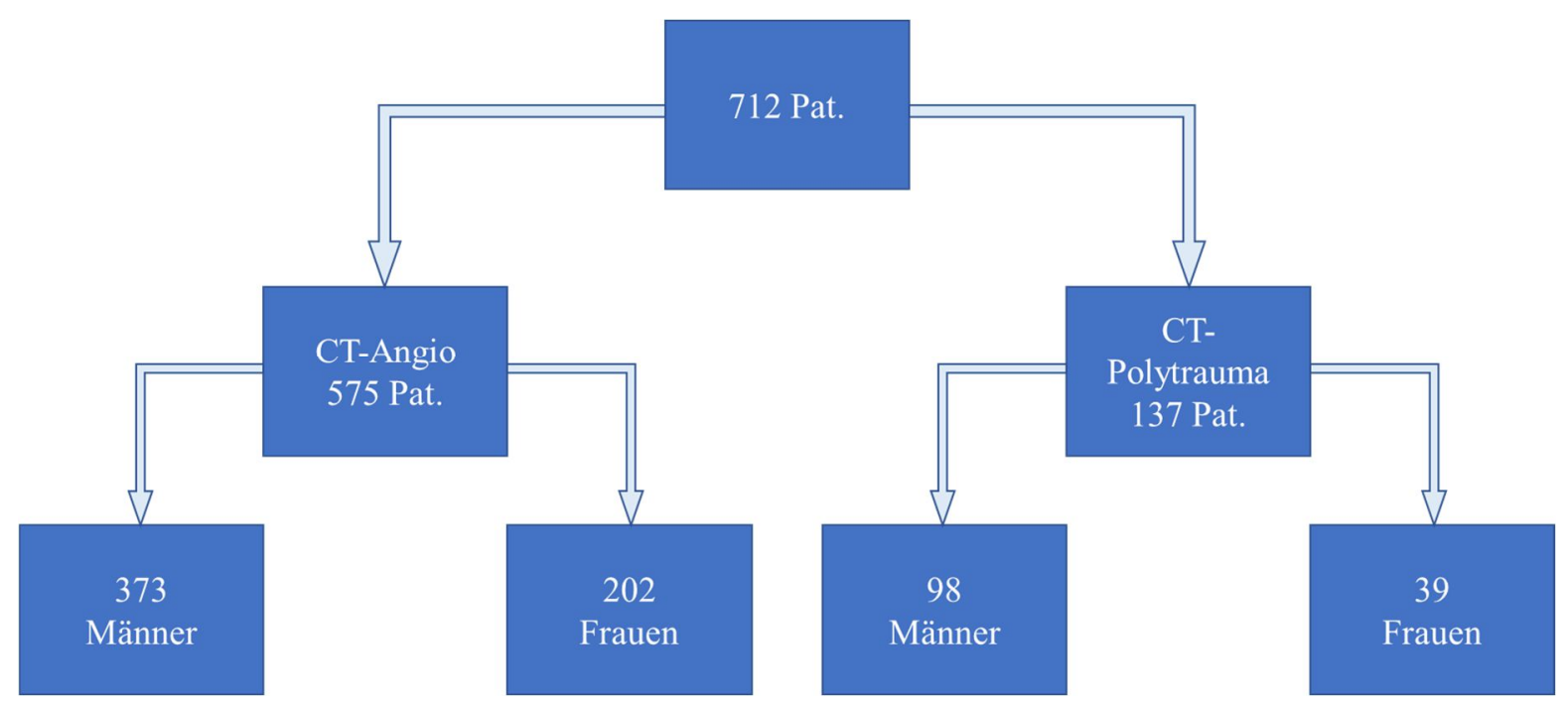

Abb. 2 Aufteilung des Studienkollektivs nach Bildgebung und Geschlecht

Aus klinischer Sicht erschien es sinnvoll, das Kollektiv in verschiedene Altersgruppen aufzuteilen. Es wurde zwischen vier Gruppen unterschieden: Patienten mit einem Alter von 1940 Jahren (1), im Alter von 40-60 Jahren (2), im Alter von 60-80 Jahren (3) und Patienten mit einem Alter $>80$ Jahren (4).

Dadurch ergibt sich eine Verteilung mit den Gruppengrößen von 153 (Gruppe 1), 210 (Gruppe 2), 356 (Gruppe 3) und 69 Patienten (Gruppe 4). Tabelle 1 spiegelt diese Verteilung wider.

Tab. 1 Aufteilung des Studienkollektivs nach Altersgruppen

\begin{tabular}{|c|c|c|}
\hline Gruppe & Alter & Anzahl an Patienten \\
\hline 1 & 19-40 Jahre & 129 \\
\hline 2 & 40-60 Jahre & 185 \\
\hline 3 & 60-80 Jahre & 334 \\
\hline 4 & >80 Jahre & 64 \\
\hline
\end{tabular}




\subsection{Messparameter}

Im Folgenden soll ein Überblick über die in der Arbeit erhobenen acetabulären und femoralen Messparameter gegeben werden (Tabelle 2).

Tab. 2 Übersicht der erhobenen Messparameter für Acetabulum und proximales Femur

\begin{tabular}{|c|c|}
\hline Acetabulum: & Femur: \\
\hline Lateraler-Center-Ecken-Winkel (LCE) & Femoral neck version (FNV) \\
\hline $\begin{array}{l}\text { Acetabuläre Anteversion in der Axialebene } \\
\text { (AcetAVa) }\end{array}$ & Centrum-Collum-Diaphysen-Winkel (CCD) \\
\hline Acetabulärer Index (AI) & 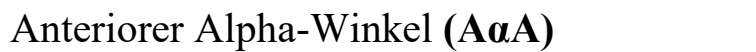 \\
\hline Sharp-Winkel (SW) & Superiorer Alpha-Winkel (SaA) \\
\hline Pfannendurchmesser (ØА) & Kopfdurchmesser (ØF) \\
\hline Acetabuläres Offset (AO) & Femorales Offset (FO) \\
\hline $\begin{array}{l}\text { Anteriorer acetabulärer } \quad \text { Sektorwinkel } \\
\text { (AASA) }\end{array}$ & \\
\hline $\begin{array}{l}\text { Posteriorer acetabulärer } \quad \text { Sektorwinkel } \\
\text { (PASA) }\end{array}$ & \\
\hline \multicolumn{2}{|c|}{ Globales Offset (GO) } \\
\hline
\end{tabular}

Des Weiteren wurde der radiologische Arthrosegrad aller Hüftgelenke nach Kellgren/Lawrence (KL) erhoben (Grad 0-4) (Kellgren und Lawrence 1957). Außerdem wurde der Quotient aus Kopfdurchmesser und Pfannendurchmesser (F/A) berechnet.

\subsubsection{LCE}

Als LCE-Winkel bezeichnet man den Winkel, der zwischen der Verbindungsachse von Hüftkopfmitte und oberem Pfannendachrand sowie der Längsachse des Körpers eingeschlossen wird. Er dient unter anderem zur Evaluierung der Überdachung des Hüftkopfes durch die Pfanne. Neben seinem prädiktiven Wert für das Pincer-Impingement $\left(>40^{\circ}\right)$, kann der LCE auch als Dysplasie-Parameter $\left(<20^{\circ}\right)$ oder als Marker für ein Zwischenstadium („Borderline“, 20-25 $5^{\circ}$ herangezogen werden (Lee und Kim 2012).

\subsubsection{AcetAVa}

Die AcetAVa beschreibt die Stellung des Acetabulums in der Sagittalebene. Positive Werte sprechen für eine Anteversion, negative Werte für Retroversion. Als pathologisch gilt ein Winkel $>15^{\circ}$ oder eine retrovertierte Ausrichtung (Stem et al. 2006). Die AcetAVa bezeichnet einen Winkel zwischen einer durch beide Pfannendachränder laufenden Graden und einer Senkrechten, welche auf den hinteren Pfannendachrand gefällt wird. 


\subsubsection{AASA und PASA}

Zur Bestimmung der axialen Überdachung des Femurkopfes werden der AASA und der PASA genutzt. Sie bezeichnen die Winkel, die zwischen der zentriert verlaufenden Verbindungsachse ausgehend von Hüftkopfmitte zum Acetabulum und dessen äußersten Ausläuferpunkten liegen. Der AASA liegt „anterior“ und der PASA ,posterior“. Winkel $<52^{\circ}$ (AASA) bzw. $<90^{\circ}$ (PASA) gelten als dysplastisch (Harrasser et al. 2016).

\subsubsection{AI}

Weiterführend ist der AI ein „Pfannendachwinkel“, der angibt, wie weit die Überdachung des Femurkopfes durch das Acetabulum in der koronaren Schnittbildgebung reicht. Er wird gebildet durch einen horizontalverlaufenden Schenkel durch den höchsten Punkt des abgebildeten Femurkopfes und einem an diesem ansetzenden Schenkel, der den tiefsten Punkt des lateralen Pfannendaches schneidet. Ein pathologischer Referenzwert für den AI findet sich in der aktuellen Literatur nicht.

\subsubsection{SW}

Der SW erfasst die Ausrichtung des Acetabulums in der koronaren Ebene (Sharp 1961). Er liegt zwischen einem waagerechten Schenkel, der den inneren Erkerpunkt schneidet und einem von dort durch den äußeren Erkerpunkt verlaufenden Schenkel. Als physiologisch gilt ein Winkel von $38-43^{\circ}$. Werte $<38^{\circ}$ werden als sogenanntes „Overcoverage“-Stadium bezeichnet. Beträgt der SW weniger als $34^{\circ}$ wird von einer schweren Ausprägungsform gesprochen. Liegt der Winkel über $43^{\circ}$ wird von einer Dysplasie ausgegangen. Der SW kann zur Bestimmung der Überdachung des Femurkopfes herangezogen werden (Tannast et al. 2015).

\subsubsection{FNV}

Die Femoral neck version ist der Antetorsionswinkel (Winkel zwischen Schenkelhalsachse und Femurkondylenebene) des Oberschenkelknochens. Der Normbereich liegt basierend auf der aktuellen Studienlage zwischen 5-25 $5^{\circ}$ Antetorsion. Werte außerhalb dieser Grenzen $\left(<5^{\circ}\right.$ [Coxa retrotorta] bzw. $>25^{\circ}$ [Coxa antetorta]) werden als pathologisch angesehen. Beim Vorliegen von negativen Werten ist von einer manifesten Retrotorsion auszugehen (Hetsroni et al. 2013).

\subsubsection{CCD}

Der CCD beschreibt einen Winkel zwischen dem Schenkelhals und dem Femurschaft. Er liegt am Schnittpunkt zweier Geraden, die durch diese Strukturen verlaufen. Wie bereits erwähnt, gelten die Coxa vara $\left(\mathrm{CCD}\right.$-Winkel $\left.<125^{\circ}\right)$ und die Coxa valga $\left(\mathrm{CCD}\right.$-Winkel $\left.>140^{\circ}\right)$ als pathologisch (Tönnis und Heinecke 1999). 


\subsubsection{A AA und $\mathrm{S} \alpha \mathrm{A}$}

Der eingangs bereits beschriebene $\alpha$-Winkels für die Detektion eines Cam-Impingements wird mit dem $A \alpha A$ gleichgesetzt. In einer Studie von Nötzli wurde neben dem $A \alpha A$ auch ein $S \alpha A$ (in der koronaren statt in der axialen Bildebene) gemessen. Der prädiktive Wert $\left(>55^{\circ}\right)$ für das Cam-Impingement bezieht sich jedoch lediglich auf den $\mathrm{A} \alpha \mathrm{A}$. Für den $\mathrm{S} \alpha \mathrm{A}$ konnte bisher keine detektierende Funktion in Bezug auf hüftmorphologische Pathologien nachgewiesen werden (Nötzli et al. 2002).

\subsubsection{AO, FO und GO}

Die Offset-Parameter sind vor allem in der Endoprothetik von Bedeutung, da anhand von ihnen die Kraftwirkung auf Hüftgelenkendoprothesen abgeschätzt werden kann (Renkawitz et al. 2011). Das FO bezeichnet den Abstand zwischen Drehzentrum des Femurkopfes und der Femurschaftachse. In Verlängerung des FO wird das AO durch die Strecke zwischen Hüftkopfdrehzentrum und Acetabulum definiert. Das GO ist die Summe aus dem FO und dem AO.

\subsubsection{Arthrosegrad}

Tabelle 3 gibt die verwendete Klassifikation des Grades der Coxarthrose nach Kellgren und Lawrence wieder.

Tab. 3 Klassifikation der Coxarthrose nach Kellgren/Lawrence

\begin{tabular}{|l|l|l|}
\hline Arthrosegrad & Klassifizierung & \\
\hline 0 & Normal & \\
\hline 1 & $\begin{array}{l}\text { Geringe subchondrale Sklerosierung. Keine Osteophyten. Keine } \\
\text { Gelenkspaltverschmälerung }\end{array}$ \\
\hline 2 & $\begin{array}{l}\text { Geringe Gelenkspaltverschmälerung. Beginnende Osteophytenbildung. } \\
\text { Angedeutete Unregelmäßigkeit der Gelenkfläche }\end{array}$ \\
\hline 3 & $\begin{array}{l}\text { Ausgeprägte Osteophytenbildung. Gelenkspaltverschmälerung. Deutliche } \\
\text { Unregelmäßigkeit der Gelenkfläche }\end{array}$ \\
\hline 4 & $\begin{array}{l}\text { Ausgeprägte Gelenkspaltverschmälerung, subchondrale Sklerosierung, } \\
\text { knöcherne Deformierungen des Hüftgelenks (Arthrosis deformans) }\end{array}$ \\
\hline
\end{tabular}




\subsection{Messmethoden}

Alle Messungen erfolgten ausnahmslos über das PACS-System (Picture Archiving and Communication System) der Universitätsmedizin Göttingen (UMG). Genutzt wurde eine Software der Firma GE Healthcare mit dem Namen Centricity ${ }^{\mathrm{TM}}$ Universal Viewer. Sämtliche Winkel und Strecken wurden mit Hilfe des Winkeltools bzw. des Streckentools gemessen.

In der koronaren Schnittbildgebung wurde zunächst eine Ebene aufgesucht, in der sowohl Hüftpfanne und Femurkopf als auch der Verlauf des Femurschaftes abgebildet waren. Im zweiten Schritt wurde über eine virtuelle Kreisschablone der Femurkopf abgegrenzt, um dessen Zentrum ermitteln zu können. Der Winkel zwischen den Strecken aus dem gefällten Lot von der Hüftpfanne ausgehend in das Zentrum des Hüftkopfes (1) sowie der Strecke beginnend im Zentrum bis hin zur äußeren Pfannendachrandgrenze ist der LCE. Alle Strecken, Halbgeraden und Geraden wurden manuell unter Nutzung der Computer-Maus gezogen. Der CCD beschreibt einen Winkel, der gemessen werden kann, wenn eine Halbgerade mittig vom Zentrum des Femurkopfes ausgehend durch den Schenkelhals (1) und eine andere mittig durch den Schaft (2) gelegt wird. Der Schnittpunkt beider Halbgeraden ist gleichzeitig der Fixationspunkt der zweiten Halbgeraden. Der S $\alpha$ A teilt sich einen Hauptschenkel mit dem CCD (1). Der andere Schenkel wird durch Strecken gebildet, die dem lateralen Übergangspunkt des Femurkopfes in den Schenkelhals entspringen und im Zentrum des Kopfes enden. Ebenfalls in dieser Bildebene gemessen wird der AI. Der Winkel liegt zwischen einer Waagerechten durch den obersten Punkt des Hüftkopfes und einer von dort aus beginnenden Halbgeraden durch die äußere untere Begrenzung des Pfannendachrandes. Abbildung 3 und 4 zeigen beispielhafte Messungen für diese Winkel. In Abbildung 3 sind LCE (Bildnummer 2), CCD (BN 3 ) und der S $\alpha$ A (BN 4) zu sehen. Abbildung 4 zeigt als einzigen Winkel den AI. 

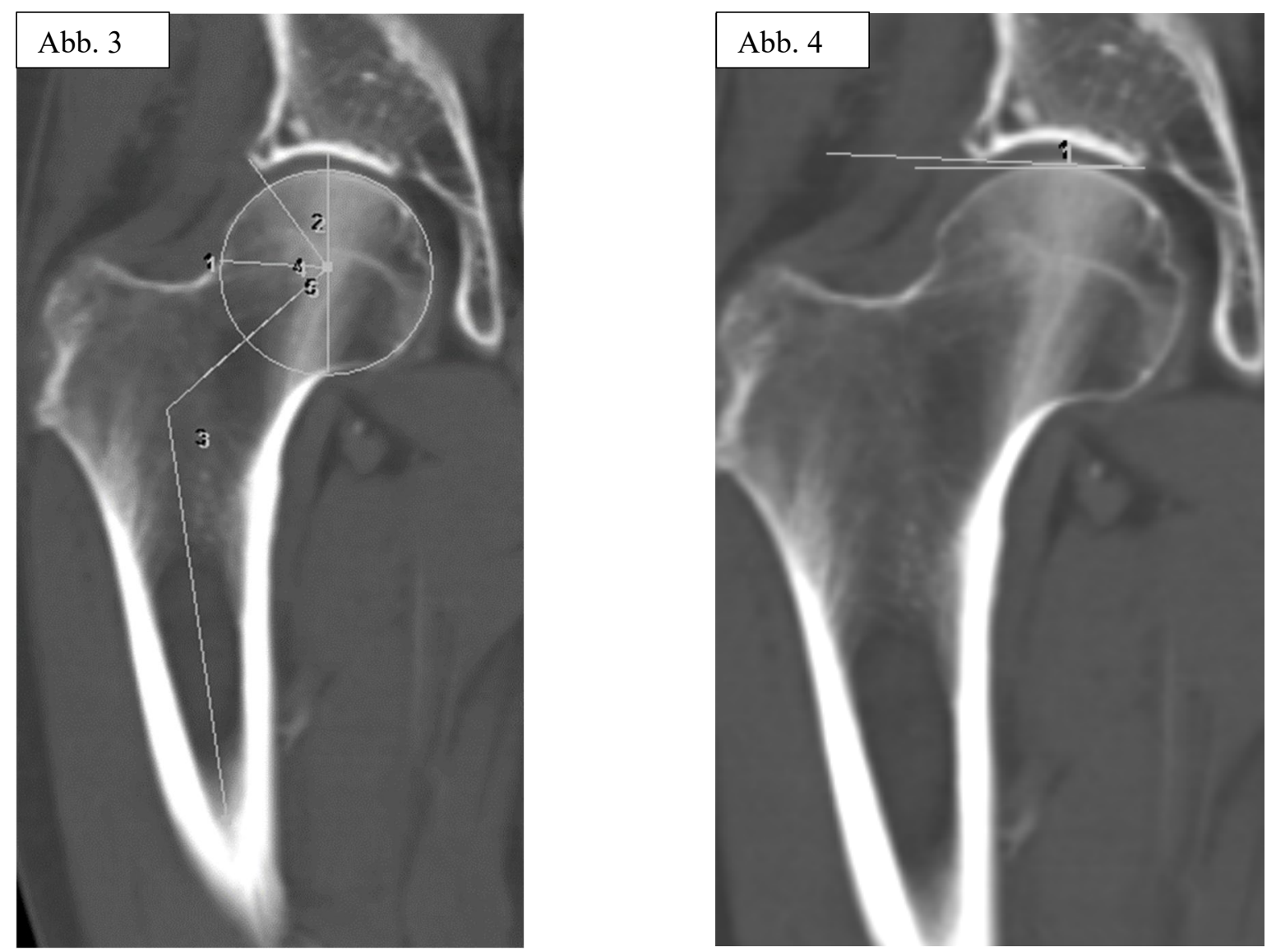

Abb. 3 und 4 Beispielhafte Darstellung der Messungen des LCEs, CCDs, SaAs (Abb. 3) und des AIs (Abb. 4)

Ebenfalls in der koronaren Schnittbildgebung wurden Arthrosegrad und die Durchmesser von Hüftpfanne sowie Hüftkopf erfasst. Die Quantifizierung des Arthrosegrads erfolgte nach den in 2.2.10 beschriebenen Kriterien. Unter Benutzung des Streckentools wurden beide Durchmesser ermittelt. Anschließend wurden beide Werte ins Verhältnis gesetzt, um den Quotienten aus Kopfdurchmesser und Pfannendurchmesser zu erhalten. Beispielhafte Messungen zeigen Abb. 5 und 6. Der Arthrosegrad in Abb. 5 wurde nach Kellgren/Lawrence als Grad 4 klassifiziert. Als manifeste Arthrose wurden Stadium 3 und 4 gewertet. In Abbildung 6 ist mit BN 1 der Pfannen- und mit BN 2 der Kopfdurchmesser bezeichnet. 

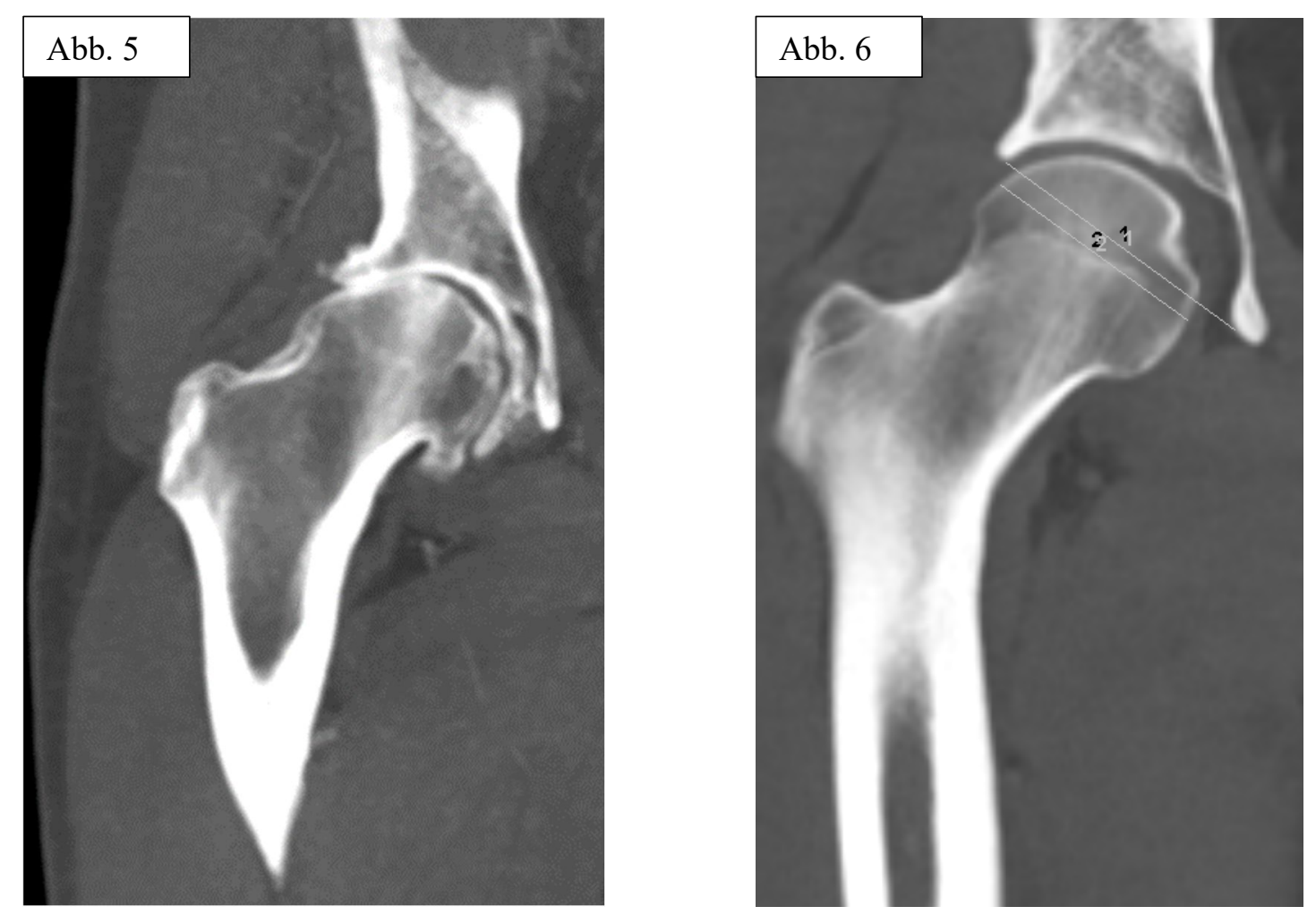

Abb. 5 und 6 Arthrosegrad 4 nach Kellgren/Lawrence (Abb. 5) und beispielhafte Darstellung der Messung des Kopf- bzw. Pfannendurchmessers (Abb. 6)

Auch die Offset-Parameter und der SW wurden in der koronaren Schnittbildebene gemessen. Zur Bestimmung des FO wurde eine Gerade durch den proximalen Femurschaft gelegt. Anschließend wurde ausgehend vom Drehzentrum des Hüftkopfes eine waagerechte Strecke bis zu der Geraden gezogen. Die Streckenlänge definiert das FO (Abb. 7). Die Strecke in entgegengesetzter Richtung (ausgehend vom Drehzentrum) bis zum Acetabulum gibt das AO an (Abb. 8). Durch Addition beider Streckenlängen ergibt sich das GO. 


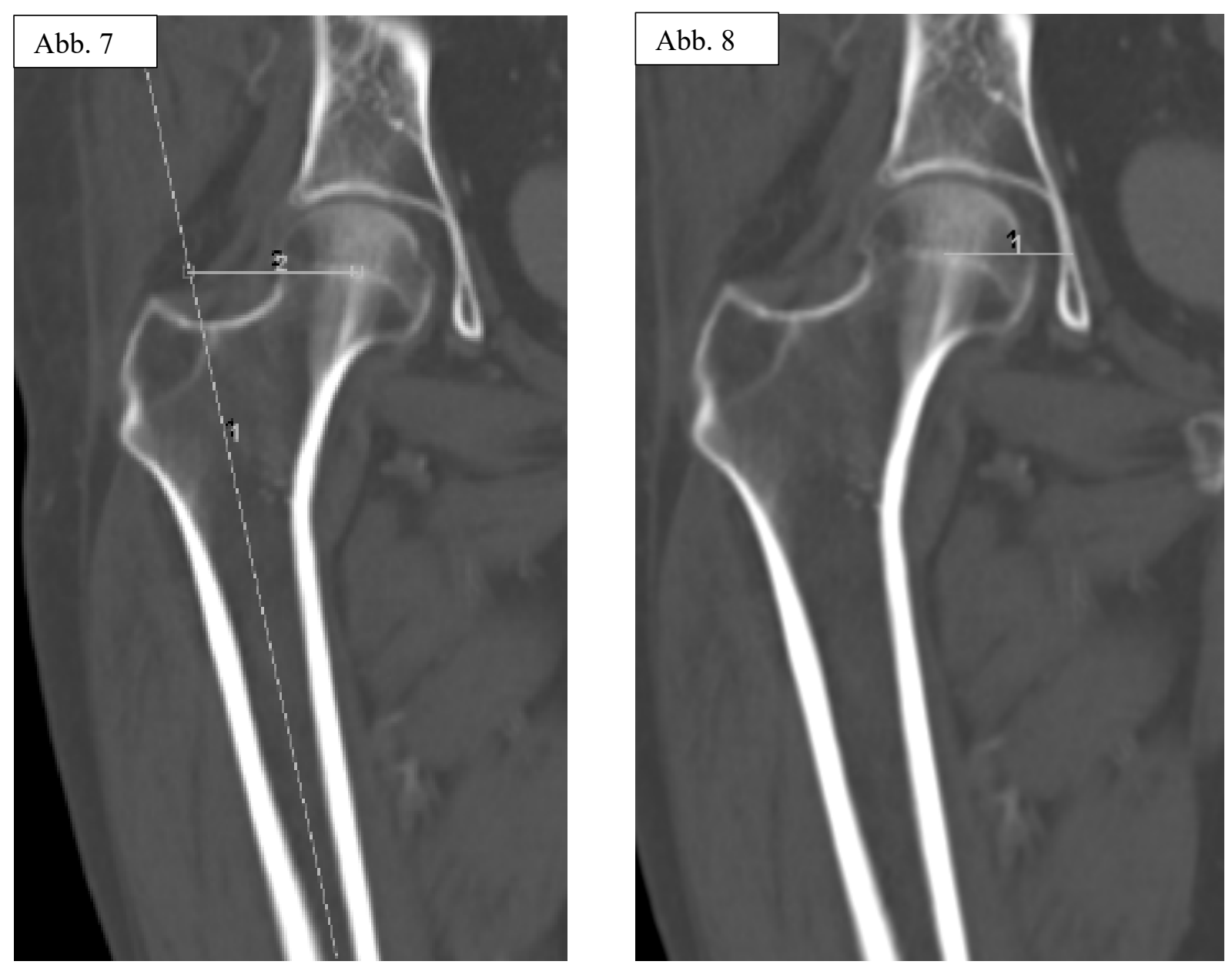

Abb. 7 und 8 Beispielhafte Darstellung der Messungen des FOs (Abb. 7) und des AOs (Abb. 8)

Zur Ermittlung des SW wurde eine Gerade in der Horizontalen durch den inneren Erkerpunkt gelegt. Letzterer diente als Fixationspunkt für den Beginn des zweiten Winkelschenkels, welcher von dort geradenförmig durch den äußeren Erkerpunkt verläuft. Der SW wurde als Winkel zwischen diesen beiden Schenkeln definiert (Abb. 9). 


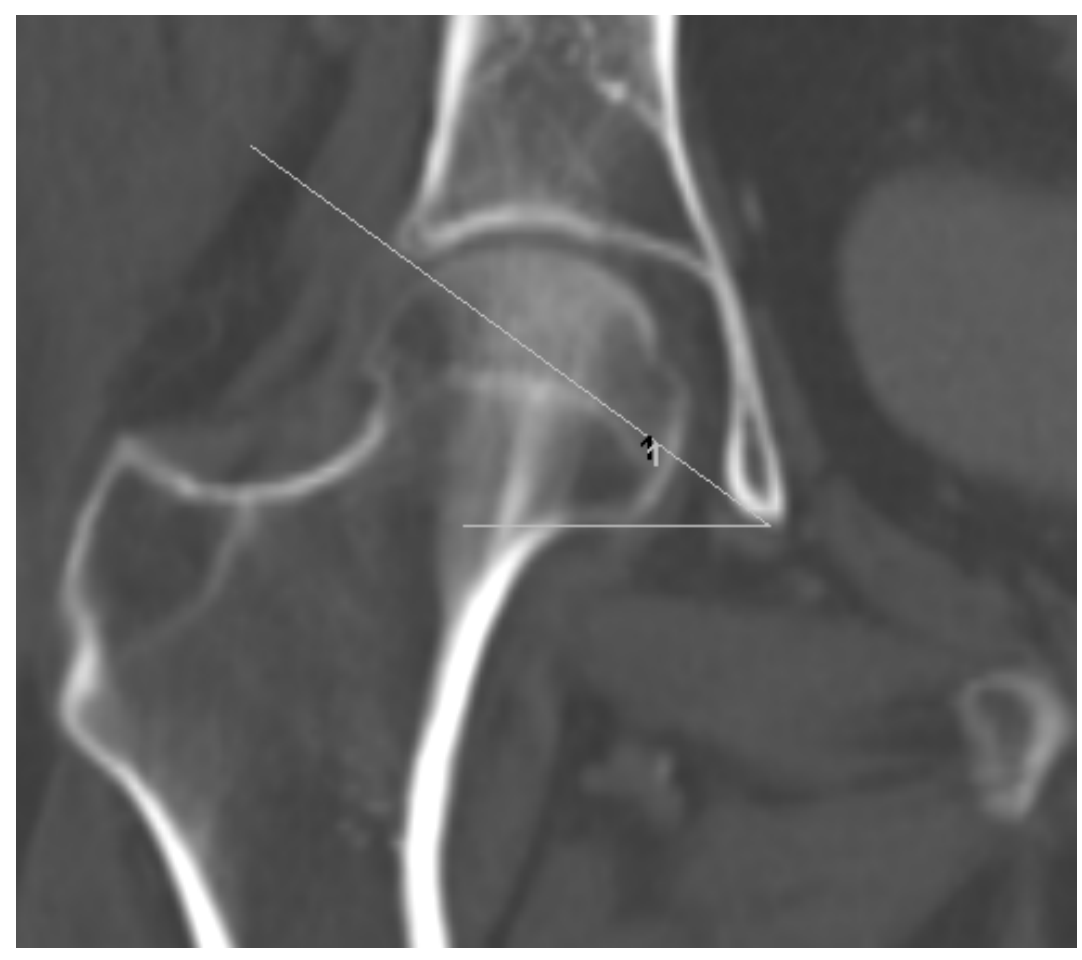

Abb. 9 Beispielhafte Darstellung der Messung des SWs

Die Erhebung der restlichen Parameter erfolgte in axialer Schnittbildgebung. Zur Erfassung von AcetAVa, AASA und PASA wurde eine Bildebene aufgesucht, in der sowohl die Pfannendachränder als auch der Femurkopf weitestgehend abgebildet waren. Die AcetAVa wurde durch den Winkel ermittelt, dessen Schenkel aus einer senkrechten Halbgeraden mit Fixationspunkt am unteren Pfannendachrand (1) und einer Strecke vom Fixationspunkt zum oberen Pfannendachrand (2) bestehen. AASA und PASA teilen sich den gleichen Hauptschenkel, der durch eine waagerechte Halbgerade mit Fixationspunkt im Zentrum des Femurkopfes gebildet wird. Der jeweils andere Schenkel ist definiert als eine Strecke vom Fixationspunkt zum oberen (AASA) bzw. zum unteren Pfannendachrand (PASA). Dieses wird in Abb. 7 nochmals verdeutlicht. BN 1 bezeichnet die AcetAVa, BN 2 den AASA und BN 3 den PASA. 


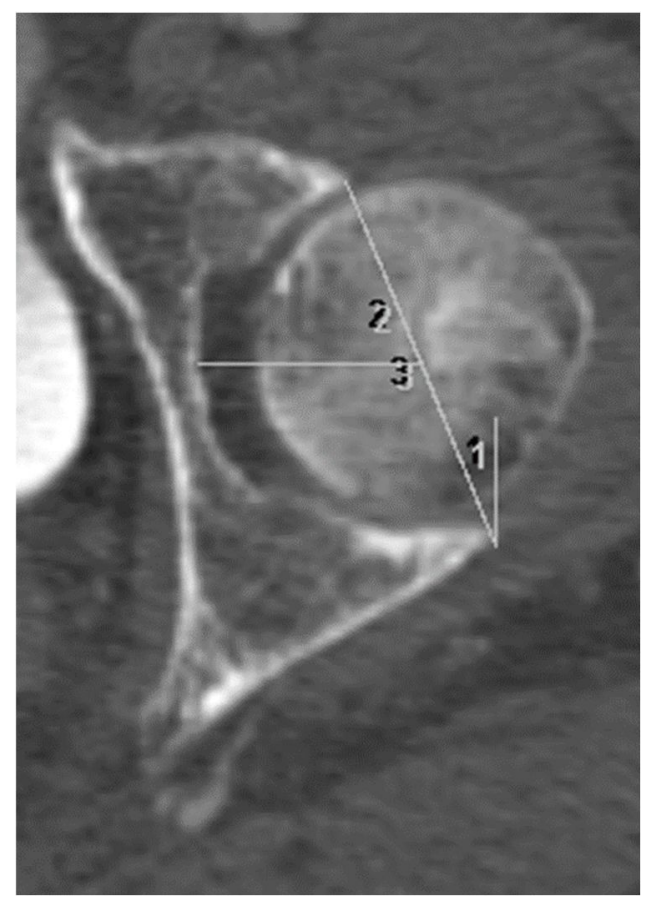

Abb. 10 Beispielhafte Darstellung der Messungen der AcetAVa, des AASAs und PASAs

Für die Messungen des A $\alpha \mathrm{A}$ und der superioren Femoral neck version (genauere Erläuterung folgt) musste ein Bildebenenwechsel erfolgen, damit die Schenkelhalskonfiguration zu bewerten war. Zunächst wurde wieder mit Hilfe der virtuellen Kreisschablone das Zentrum des Femurkopfes ermittelt. Auch A $\alpha$ A besitzt einen Hauptschenkel, der von einer Halbgeraden mit Fixationspunkt im Zentrum mittig durch den Schenkelhals verlaufend gebildet wird. Der andere Schenkel ist analog zu dem superioren Winkel definiert als Strecke ausgehend vom Fixationspunkt zum lateralen Übergangspunkt zwischen Femurkopf und Schenkelhals. Die Strecke oberhalb des Hauptschenkels gehört zum Winkel des A $\alpha$ A.

Die Femoral neck version muss durch zwei gemessene Werte ermittelt werden. Sie ergibt sich aus einem superioren und einem inferioren Wert. Der superiore Wert wird in derselben Bildebene bestimmt wie der A $\alpha$ A. Er ergibt sich aus dem Winkel, der zwischen einer Geraden mittig durch den Schenkelhals und einer Horizontalen liegt. Abb. 8 spiegelt diese Messungen wider. BN 2 zeigt den A $\alpha$ A und BN 4 die superiore Femoral neck version. 


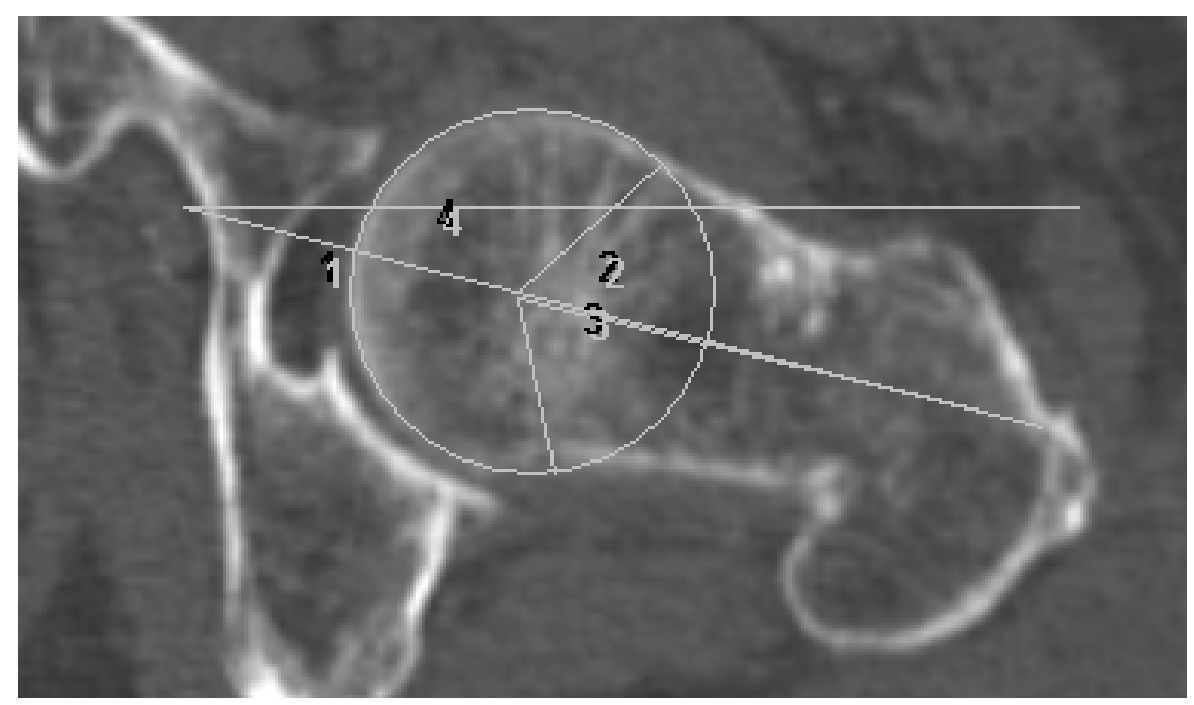

Abb. 11 Beispielhafte Darstellung der Messungen des AaAs und der superioren Femoral neck version

Zur Messung der inferioren Femoral neck version war ein weiterer Bildebenenwechsel erforderlich. In dieser Ebene müssen beide Femurkondylen abgebildet sein. Der zu bestimmende Winkel wird gebildet durch einen Schenkel, welcher als Strecke zwischen den äußersten Mittelpunkten beider Kondylen liegt und einem Schenkel, der als Halbgerade mit Fixationspunkt auf einem Kondylenmittelpunkt, fortlaufend in Richtung des anderen, verläuft. Eine solche Messung zeigt Abb. 9.

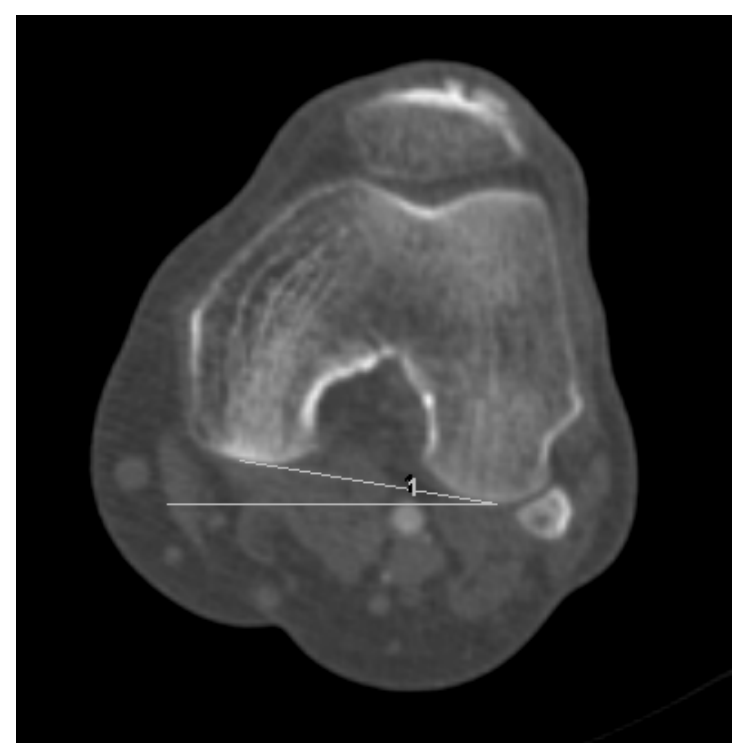

Abb. 12 Beispielhafte Darstellung der Messung der inferioren Femoral neck version 
Der Gesamtwert der FNV ist abhängig von der lageabhängigen Kondylenrotation. Liegt eine Innenrotationsstellung des distalen Femurs vor, werden beide Werte addiert. Bei einer festgestellten Außenrotation des distalen Femurs wird von der superioren FNV die inferiore subtrahiert.

Um lagerungsbedingte Messfehler zu vermeiden, wurde weiterhin die Beckenverkippung bestimmt. Dazu wurde eine Gerade zwischen den beiden Os sacrum Spitzen gezogen (gemessen in der koronaren Bildebene) und der Winkel zwischen dieser und einer Horizontalen gemessen (Abb. 13).

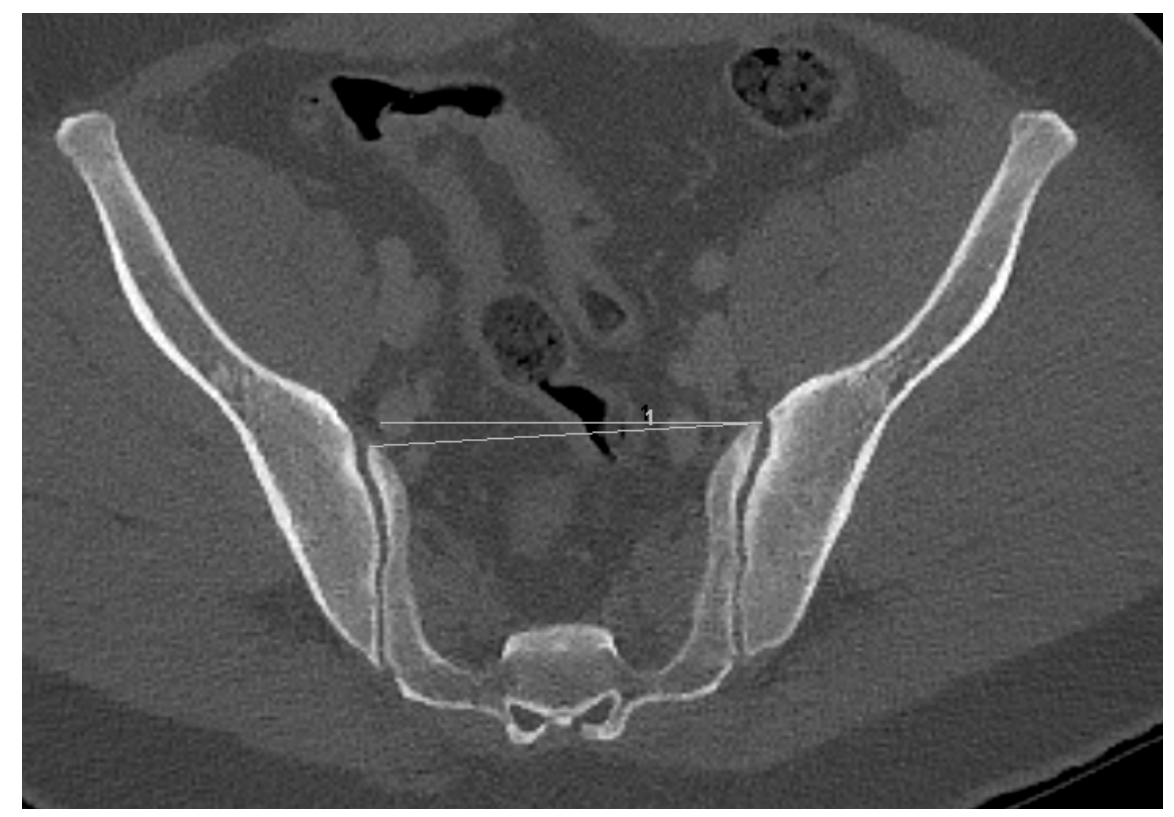

Abb. 13 Beispielhafte Darstellung der Messung der Beckenverkippung in der Koronarebene

Lagerungsabhängige Parameter sind der LCE, der AI und der SW in der Koronarebene sowie die AcetAVa, der AASA und der PASA in der Axialebene. Nach einem festen Schema wurde der Verkippungswinkel je nach Ausrichtung (Verkippung nach rechts oder links) entweder addiert oder subtrahiert. Anhand von Tabelle 4 und 5 lässt sich der Messausgleich nachvollziehen. 
Tab. 4 Messausgleich der Parameter in der Koronarebene

\begin{tabular}{|c|c|c|}
\hline & links & rechts \\
\hline Verkippung nach links & $\mathrm{LCE} \downarrow$ & $\mathrm{LCE} \uparrow$ \\
& $\mathrm{AI} \uparrow$ & $\mathrm{AI} \downarrow$ \\
& $\mathrm{SW} \uparrow$ & $\mathrm{SW} \downarrow$ \\
\hline Verkippung nach rechts & $\mathrm{LCE} \uparrow$ & $\mathrm{LCE} \downarrow$ \\
& $\mathrm{AI} \downarrow$ & $\mathrm{AI} \uparrow$ \\
& $\mathrm{SW} \downarrow$ & $\mathrm{SW} \uparrow$ \\
\hline
\end{tabular}

( $\uparrow=$ addiert; $\downarrow=$ subtrahiert $)$

Tab. 5 Messausgleich der Parameter in der Axialebene

\begin{tabular}{|c|c|c|}
\hline & links & rechts \\
\hline Verkippung nach links & AcetAVa $\uparrow$ & AcetAVa $\downarrow$ \\
& AASA $\downarrow$ & AASA $\uparrow$ \\
& PASA $\uparrow$ & PASA $\downarrow$ \\
\hline Verkippung nach rechts & AcetAVa $\downarrow$ & AcetAVa $\uparrow$ \\
& AASA $\uparrow$ & AASA $\downarrow$ \\
& PASA $\downarrow$ & PASA $\uparrow$ \\
\hline
\end{tabular}

$(\uparrow=$ addiert; $\downarrow=$ subtrahiert $)$

\subsection{Statistische Methoden}

Alle statistischen Analysen wurden mit dem Source-Statistik-Programm „R“ (R Core Development Team, Vienna, Austria) and RStudio (RStudio Inc., Boston, MA, USA) sowie dem Tabellenkalkulationsprogramm „Excel“ (Microsoft Corporation, Redmond, WA, USA) durchgeführt. Für die deskriptive Statistik wurden als kontinuierliche Variable Mittelwerte mit den zugehörigen Standardabweichungen bestimmt. Als kategoriale Variable wurden absolute Zahlen und Prozentbeträge angegeben.

Ein $\alpha$-Wert von 0,05 wurde als statistisch signifikant angesehen. Alle angegebenen $p$-Werte wurden zweiseitig erhoben. Zur Analyse der Werte der kontinuierlichen Variablen wurde der „Wilcoxon rank sum test“ (= Mann-Whitney-U Test) implementiert. Durch diesen sollte die Signifikanz der Verteilung eines einzelnen Messparameters gegenüber der Gesamtheit der Werte derselben Kategorie überprüft werden. 
Für den Seitenvergleich des rechten und linken Hüftgelenkes wurde der „Wilcoxon signed rank test“" (sogenannter ,paired test") angewendet. Der Wilcoxon Test kam zum Einsatz, da das untersuchte Kollektiv der vorliegenden Studie keine normalverteilten Altersgruppen aufweist (,voraussetzungsfreies Verfahren“). Die Bedingungen einer ordinalskalierten Datenerhebung werden erfüllt. Um einen Vergleich der gemessenen Werte für den Arthrosegrad nach Kallgren/Lawrence anzustellen (kategoriale Variable) wurde der „chi-square test“ genutzt. Durch den Verteilungstest wurde ermittelt, ob sich die Messwerte auf eine bestimmte Art verteilen (Gaus und Muche 2017). 


\section{Ergebnisse}

\subsection{Datenverteilung der erfassten Parameter und Seitenvergleich}

Wie unter 2.3 dargestellt, wurden für alle kontinuierlichen Variablen die Mittelwerte mit den zugehörigen Standardabweichungen und p-Werten bestimmt sowie für den Arthrosegrad (kategoriale Variable) absolute Zahlen und Prozentwerte ermittelt. Die Tabellen 6 und 7 geben sämtliche Werte wieder.

Tab. 6 Mittelwerte der femoralen und acetabulären Parameter im Gesamtkollektiv sowie in Abhängigkeit von der Seite

\begin{tabular}{|c|c|c|c|c|c|}
\hline & & $\begin{array}{l}\text { gesamt } \\
n=1424\end{array}$ & $\begin{array}{c}\text { links } \\
\mathbf{n}=712\end{array}$ & $\begin{array}{l}\text { rechts } \\
n=712\end{array}$ & p-Werte \\
\hline LCE & {$\left[\right.$ in $\left.^{\circ}\right]$} & $40,9( \pm 8,6)$ & $42,9( \pm 8,6)$ & $38,9( \pm 8,3)$ & $<0,0001$ \\
\hline AI & {$\left[\right.$ in $\left.^{\circ}\right]$} & $0,1( \pm 5,8)$ & $0,1( \pm 5,6)$ & $0,0( \pm 5,9)$ & 0,87 \\
\hline AcetAVa & {$\left[\right.$ in $\left.^{\circ}\right]$} & $20,5( \pm 6,7)$ & $20,3( \pm 6,7)$ & $20,8( \pm 6,8)$ & 0,17 \\
\hline AASA & {$\left[\right.$ in $\left.^{\circ}\right]$} & $65,9( \pm 9,2)$ & $65,6( \pm 9,4)$ & $65,2( \pm 9,0)$ & 0,31 \\
\hline PASA & {$\left[\right.$ in $\left.^{\circ}\right]$} & $106,8( \pm 12,9)$ & $106,0( \pm 13,1)$ & $107,6( \pm 12,8)$ & 0,007 \\
\hline CCD & {$\left[\mathrm{in}^{\circ}\right]$} & $130,2( \pm 8,4)$ & $132,0( \pm 8,2)$ & $128,4( \pm 8,1)$ & $<0,0001$ \\
\hline $\mathrm{S} \alpha \mathrm{A}$ & {$\left[\right.$ in $\left.^{\circ}\right]$} & $46,1( \pm 8,5)$ & $44,9( \pm 8,1)$ & $47,3( \pm 8,6)$ & $<0,0001$ \\
\hline $\mathrm{A} \alpha \mathrm{A}$ & {$\left[\right.$ in $\left.^{\circ}\right]$} & $41,2( \pm 7,6)$ & $39,3( \pm 7,3)$ & $43,2( \pm 7,4)$ & $<0,0001$ \\
\hline FNV & {$\left[\mathrm{in}^{\circ}\right]$} & $11,7( \pm 10,0)$ & $12,1( \pm 10,0)$ & $11,3( \pm 10,1)$ & 0,11 \\
\hline SW & {$\left[\mathrm{in}^{\circ}\right]$} & $37,1( \pm 4,8)$ & $36,6( \pm 4,8)$ & $37,6( \pm 4,9)$ & 0,0003 \\
\hline FO & [in $\mathrm{mm}$ ] & $40,0( \pm 8,1)$ & $39,0( \pm 7,9)$ & $41,0( \pm 8,2)$ & $<0,0001$ \\
\hline $\mathrm{AO}$ & [in $\mathrm{mm}$ ] & $31,8( \pm 4,5)$ & $31,5( \pm 4,4)$ & $32,2( \pm 4,5)$ & 0,009 \\
\hline GO & [in $\mathrm{mm}$ ] & $71,8( \pm 9,7)$ & $70,5( \pm 9,3)$ & $73,2( \pm 9,8)$ & $<0,0001$ \\
\hline$\varnothing \mathrm{F}$ & [in $\mathrm{mm}$ ] & $47,6( \pm 4,1)$ & $47,8( \pm 4,0)$ & $47,4( \pm 4,1)$ & 0,16 \\
\hline ØА & [in $\mathrm{mm}$ ] & $58,5( \pm 5,3)$ & $58,4( \pm 5,3)$ & $58,5( \pm 5,3)$ & 0,48 \\
\hline $\mathrm{F} / \mathrm{A}$ & [in \%] & $81,6( \pm 0,1)$ & $82,0( \pm 0,1)$ & $81,2( \pm 0,1)$ & 0,001 \\
\hline
\end{tabular}

Tab. 7 Verteilung der Arthrosewerte (nach Kellgren/Lawrence) im Gesamtkollektiv und nach Seitenunterschieden

\begin{tabular}{|c|c|c|c|c|}
\hline & $\begin{array}{c}\text { gesamt } \\
\mathbf{n = 1 4 2 4}\end{array}$ & $\begin{array}{c}\text { links } \\
\mathbf{n = 7 1 2}\end{array}$ & $\begin{array}{c}\text { rechts } \\
\mathbf{n = 7 1 2}\end{array}$ & p-Werte \\
\hline Grad & & & & 0,46 \\
0 & $189(13,3 \%)$ & $87(12,2 \%)$ & $102(14,3 \%)$ & \\
1 & $472(33,2 \%)$ & $231(32,4 \%)$ & $241(33,9 \%)$ & \\
2 & $673(47,3 \%)$ & $352(49,4 \%)$ & $321(45,1 \%)$ & \\
3 & $77(5,4 \%)$ & $37(5,2 \%)$ & $40(5,6 \%)$ & \\
4 & $13(0,9 \%)$ & $5(0,7 \%)$ & $8(11 \%)$ & \\
\hline
\end{tabular}


Die hier dargestellten Daten beantworten die erste und die zweite Fragestellung der vorliegenden Arbeit. Die Messungen der Parameter ergaben folgende Mittelwerte für die insgesamt 1424 untersuchten Hüften:

LCE: Für den LCE ergab sich ein Mittelwert von 40,9 $( \pm 8,6)$, wobei die linke Hüftseite im Mittel einen signifikant größeren Winkel $\left(42,9^{\circ}[ \pm 8,6]\right)$ als die rechte $\left(38,9^{\circ}[ \pm 8,3]\right)$ aufwies $(\mathrm{p}<0,0001)$.

AI: Der AI wies einen Mittelwert von $0,1^{\circ}( \pm 5,8)$ auf. Die seitenbezogenen Werte wichen um lediglich $0,1^{\circ}$ von diesen ab (rechts) bzw. waren identisch (links). Die Messwerte waren seitenabhängig nicht signifikant unterschiedlich $(\mathrm{p}=0,87)$.

AcetAVa: Die Analyse der 1424 Hüften ergab einen durchschnittlichen Wert von $20,5^{\circ}( \pm 6,7)$ für die AcetAVa. Es wurden keine signifikanten $(p=0,17)$ Seitendifferenzen gefunden (links $20,3^{\circ}[ \pm 6,7]$, rechts $\left.20,8^{\circ}[ \pm 6,8]\right)$.

AASA: Die Mittelwerte für den AASA betrug 65,9 $9^{\circ}(99,2)$. Linksseitig wurde ein Winkel von $65,6^{\circ}( \pm 9,4)$ ermittelt und rechtsseitig ein Winkel von $65,2^{\circ}( \pm 9,0)$. Es bestand keine Seitendifferenz $(\mathrm{p}=0,31)$.

PASA: Der PASA-Winkel zeigte im Mittel einen Wert von $106,8^{\circ}( \pm 12.9)$. Für die linke Hüftseite lag er bei $106,0^{\circ}( \pm 13,1)$, für die rechte bei $107,6^{\circ}( \pm 12.8)$. Es konnte ein signifikanter Seitenunterschied gemessen werden $(p=0,007)$.

CCD: Der Mittelwert betrug 130, $2^{\circ}( \pm 8,4)$. Der CCD-Winkel war im linken Hüftgelenk 132, $0^{\circ}$ $( \pm 8,2)$ und im rechten $128,4^{\circ}( \pm 8,1)$. Es konnte eine signifikante Seitendifferenz gemessen werden $(p-$ Wert $<0,0001)$.

SaA: Für den $\mathrm{S} \alpha \mathrm{A}$ ergab sich ein Gesamtmittelwert von $46,1^{\circ}( \pm 8,5)$. Linksseitig wurde ein Winkel von $44,9^{\circ}( \pm 8,1)$ und rechtsseitig von $47,3^{\circ}( \pm 8,6)$ gemessen. Es zeigte sich ein signifikanter Seitenunterschied ( $\mathrm{p}$-Wert<0,0001).

AaA: Beim A $\alpha$ A konnte im Mittel ein Winkel von $41,2^{\circ}( \pm 7,6)$ festgestellt werden. Die Abweichungen der beiden Hüftseiten von diesem Wert betrugen jeweils ca. $2^{\circ}$ (links 39,3 $[ \pm 7,3]$, rechts $\left.43,2^{\circ}[ \pm 7,4]\right)$. Die Auswertungen ergaben einen signifikanten Seitenunterschied in Bezug auf den $\mathrm{A} \alpha \mathrm{A}$ (p-Wert<0,0001).

FNV: Die FNV wies einen Mittelwert von $11,7^{\circ}( \pm 10,0)$ auf (links $12,1^{\circ}[ \pm 10,0]$ und rechts $\left.11,3^{\circ}[ \pm 10,1]\right)$. Es konnten keine signifikanten Unterschiede festgestellt werden $(p=0,11)$. 
SW: Der Sharp-Winkel präsentierte sich im Mittel mit 37, $1^{\circ}( \pm 4,8)$. Der p-Wert betrug 0,0003 und die signifikanten Seitenunterschiede wichen um jeweils $0,5^{\circ}$ vom Gesamtmittelwert ab (links $36,6^{\circ}[ \pm 4,8]$, rechts $37,6^{\circ}[ \pm 4,9]$ ).

Für alle drei Offset-Parameter konnten signifikante Seitendifferenzen ermittelt werden (p-Werte: FO: <0,0001, AO: 0,009, GO: <0,0001). Die einzelnen Messergebnisse werden in der Folge beschrieben.

FO: Das femorale Offset zeigte einen Mittelwert von 40,0 mm $( \pm 8,1)$, wobei sich Werte von $39,0 \mathrm{~mm}( \pm 7,9)$ in den linken Hüften und von 41,0 $\mathrm{mm}( \pm 8,2)$ in den rechten Hüften fanden.

AO: Für das acetabuläre Offset konnte im Mittel ein Wert von 31,8 mm $( \pm 4,5)$ detektiert werden (links 31,5 $\mathrm{mm}[ \pm 4,4]$, rechts $32,2[ \pm 4,5]$ ).

GO: Bedingt durch die Ergebnisse von FO und AO konnte für das globale Offset linksseitig ein Mittelwert von 70,5 $\mathrm{mm}( \pm 9,3)$ und rechtsseitig von 73,2 $\mathrm{mm}( \pm 9,8)$ ermittelt werden. Daraus resultierte ein Gesamtmittelwert von $71,8 \mathrm{~mm}( \pm 9,7)$.

Es konnten keine signifikanten Seitenunterschiede für ØF $(p=0,16)$ und $\varnothing A(p=0,48)$ gefunden werden. Das Verhältnis aus beiden Parametern (F/A) wies Seitendifferenzen aus $(p=0,001)$. Die einzelnen Messergebnisse präsentierten sich wie folgt:

ØF: Der Femurkopfdurchmesser zeigte einen Mittelwert von 47,6 mm $( \pm 4,1)$, mit seitenabhängigen Abweichungen von $0,2^{\circ}$ (links 47,8 $\mathrm{mm}[ \pm 4,0]$, rechts 47,4 $\left.\mathrm{mm}\left[ \pm 4,1^{\circ}\right]\right)$.

ØA: Beim Pfannendurchmesser ergab sich im Mittel mit einem Wert von 58,5 mm $( \pm 5,3)$. In den linken Hüftgelenken betrug dieser 58,4 mm $( \pm 5,3)$ und in den rechten 58,5 mm $( \pm 5,3)$.

F/A: Das Verhältnis vom Femurkopfdurchmesser zum Pfannendurchmesser erbrachte für alle 1424 Hüften einen Mittelwert von 81,6\% $( \pm 0,1)$. Linksseitig wurden 82,0\% $( \pm 0,1)$ ermittelt und rechtsseitig $81,2 \%( \pm 0,1)$.

Die Auswertung des Arthrosegrades nach Kellgren/Lawrence zeigte keine signifikanten Seitenunterschiede $(p=0,46)$. In der nachfolgenden Darstellung beziehen sich die seitenbezogenen Prozentangaben immer auf ein n von 712 (712 linke und 712 rechte Hüften) und die totalen Ergebnisse immer auf ein n von 1424 (Anzahl von linken und rechten Hüften zusammen). 
Grad 0: Insgesamt wurden 189 (13,3\%) Hüften ohne Anzeichen für Arthrose gefunden (links $87(12,2 \%)$, rechts $102(14,3 \%))$.

Grad 1: Leichte Arthrosezeichen zeigten sich bei 472 (33,2\%) Gelenken, wovon 231 (32,4\%) linksseitig auftraten und $241(33,9 \%)$ rechtsseitig.

Grad 2: In Stadium 2 lagen 673 (47,3\%) Hüften vor (links 352 [49,4\%], rechts 321 [45,1\%]).

Grad 3: Manifeste radiologische Arthrosen wurden in 37 (5,2\%) linken Hüftgelenken und 40 (5,6\%) rechten Hüftgelenken gefunden (KL 3). Daraus folgte eine Gesamtzahl von 77 (5,4\%) Hüften in Stadium 3.

Grad 4: Das radiologische Endstadium einer Arthrose (KL 4) trat 13 Mal auf (0,9\%). 5 solcher Messungen fanden sich linksseitig $(0,7 \%)$ und $8(1,1 \%)$ rechtsseitig.

\subsection{Spezifische Verteilungsbesonderheiten}

\subsubsection{Altersunterschiede}

Die Darstellung der Altersunterschiede beantwortet die dritte Fragestellung der vorliegenden Arbeit. Die Ergebnisse zeigen Tabelle 8 und 9.

Tab. 8 Verteilung der Mittelwerte für femorale und acetabuläre Parameter nach Altersgruppen

\begin{tabular}{|c|c|c|c|c|c|c|}
\hline & & $\begin{array}{c}19-40 \text { LJ. } \\
n=258\end{array}$ & $\begin{array}{c}\text { 40-60 LJ. } \\
n=370\end{array}$ & $\begin{array}{c}\text { 60-80 LJ. } \\
n=668\end{array}$ & $\begin{array}{c}>80 \mathrm{LJ} . \\
\mathrm{n}=128\end{array}$ & p-Werte \\
\hline$\overline{\mathrm{LCE}}$ & {$\left[\right.$ in $\left.^{\circ}\right]$} & $34,8( \pm 7,3)$ & $40,6( \pm 8,6)$ & $43,0( \pm 7,7)$ & $44,0( \pm 9,6)$ & 0,0001 \\
\hline AI & {$\left[\right.$ in $\left.^{\circ}\right]$} & $2,8( \pm 5,3)$ & $0,1( \pm 5,9)$ & $-0,9( \pm 5,7)$ & $-0,7( \pm 5,4)$ & $<0,0001$ \\
\hline AcetAVa & {$\left[\right.$ in $\left.{ }^{\circ}\right]$} & $18,5( \pm 5,9)$ & $20,4( \pm 6,5)$ & $20,9( \pm 7,0)$ & $23,3( \pm 6,5)$ & $<0,0001$ \\
\hline AASA & {$\left[\right.$ in $\left.{ }^{\circ}\right]$} & 63,4 & $8,8)$ & $9,2)$ & $65,7( \pm 10,2)$ & 0001 \\
\hline PASA & {$\left[\right.$ in $\left.^{\circ}\right]$} & $100,0( \pm 11,2)$ & $106,0( \pm 12,1)$ & $108,7( \pm 12,8)$ & $112,6( \pm 13,7)$ & $<0,0001$ \\
\hline $\mathrm{CCD}$ & {$\left[\right.$ in $\left.^{\circ}\right]$} & $131,4( \pm 8,5)$ & $131,3( \pm 7,9)$ & $129,4( \pm 8,3)$ & $128,9( \pm 9,3)$ & $<0,0001$ \\
\hline $\mathrm{S} \alpha \mathrm{A}$ & {$\left[\right.$ in $\left.^{\circ}\right]$} & $47,0( \pm 8,2)$ & $46,0( \pm 8,3)$ & $8,5)$ & 46,2 & 0,21 \\
\hline $\mathrm{A} \alpha \mathrm{A}$ & {$\left[\right.$ in $\left.{ }^{\circ}\right]$} & $38,9( \pm 7,6)$ & 41,9 & $7,5)$ & 39,3 & $<0,0001$ \\
\hline FNV & {$\left[\right.$ in $\left.{ }^{\circ}\right]$} & $11,4( \pm 11,5)$ & $11,8( \pm 10,2)$ & $11,7( \pm 9,6)$ & $12,3( \pm 8,2)$ & 0,85 \\
\hline SW & {$\left[\right.$ in $\left.{ }^{\circ}\right]$} & $40,2( \pm 4,3)$ & $37,0( \pm 4,4)$ & $36,3( \pm 4,5)$ & $35,5( \pm 5,2)$ & $<0,0001$ \\
\hline $\mathrm{FO}$ & {$[$ in $\mathrm{mm}]$} & 37,6 & 39 & $3,1)$ & 41,6 & 0001 \\
\hline $\mathrm{AO}$ & {$[$ in $\mathrm{mm}]$} & $31,2($ & 32,0 & $32,3( \pm 4,6)$ & 30,4 & $<0,0001$ \\
\hline GO & {$[$ in $\mathrm{mm}]$} & $68,7( \pm 8,7)$ & $71,0( \pm 9,7)$ & $73,5( \pm 9,7)$ & $71,9( \pm 9,3)$ & $<0,0001$ \\
\hline$\varnothing \mathrm{F}$ & {$[$ in $\mathrm{mm}]$} & $46,2( \pm 3,6)$ & & $48,4( \pm 4,1)$ & $47,3( \pm 4,0)$ & $<0,0001$ \\
\hline$\varnothing \mathrm{A}$ & {$[$ in $\mathrm{mm}]$} & $58,1( \pm 4,6)$ & $57,8( \pm 5,3)$ & $5,4)$ & $57,9( \pm 5,7)$ & 0,0006 \\
\hline F/A & {$[$ in $\%]$} & $79,6( \pm 0,0)$ & $82,0( \pm 0,1)$ & $82,0( \pm 0,1)$ & $82,0( \pm 0,1)$ & $<0,0001$ \\
\hline
\end{tabular}


Tab. 9 Verteilung der Arthrosewerte (nach Kellgren/ Lawrence) nach Altersgruppen

\begin{tabular}{|c|c|c|c|c|c|}
\hline & $\begin{array}{c}\mathbf{1 9 - 4 0} \text { LJ. } \\
\mathbf{n = 2 5 8}\end{array}$ & $\begin{array}{c}\mathbf{4 0 - 6 0} \text { LJ. } \\
\mathbf{n = 3 7 0}\end{array}$ & $\begin{array}{c}\mathbf{6 0 - 8 0} \text { LJ. } \\
\mathbf{n = 6 6 8}\end{array}$ & $\begin{array}{c}>\mathbf{8 0} \text { LJ. } \\
\mathbf{n = 1 2 8}\end{array}$ & p-Werte \\
\hline $\mathrm{Grad}$ & & & & & $<0,0001$ \\
0 & $96(37,2 \%)$ & $37(10,0 \%)$ & $56(8,4 \%)$ & $0(0,0 \%)$ & \\
1 & $93(36,1 \%)$ & $139(37,6 \%)$ & $210(31,4 \%)$ & $30(23,4 \%)$ & \\
2 & $69(26,7 \%)$ & $182(49,2 \%)$ & $353(52,8 \%)$ & $69(53,9 \%)$ & \\
3 & $0(0,0 \%)$ & $9(2,4 \%)$ & $44(6,6 \%)$ & $24(18,8 \%)$ & \\
4 & $0(0,0 \%)$ & $3(0,8 \%)$ & $5(0,8 \%)$ & $5(3,9 \%)$ & \\
\hline
\end{tabular}

Nachfolgend werden die den Tabellen entnehmbaren Informationen dargestellt.

Die Ergebnisse wurden nach Altersgruppen gegliedert. Die Aufteilung erfolgte in vier Gruppen (nachfolgend mit „,G“) abgekürzt: G1 19-40 Jahre, G2 40-60 Jahre, G3 60-80 Jahre und G4 >80 Jahre.

LCE: Der LCE zeigte eine mit dem Alter steigende Winkelgröße, beginnend bei $34,8^{\circ}( \pm 7,3)$ in G1, über $40,6^{\circ}( \pm 8,6)$ in $\mathrm{G} 2$ und $43,0^{\circ}( \pm 7,7)$ in $\mathrm{G} 3$, bis hin zu $44,0^{\circ}( \pm 9,6)$ in G4. Die Unterschiede zwischen den Altersgruppen waren signifikant $(\mathrm{p}<0,0001)$.

AI: Beim AI-Winkel konnte eine Abnahme der Winkelgröße, ausgehend vom höchsten Wert in G1, gemessen werden. Dort betrug der AI $2,8^{\circ}( \pm 5,3)$. G2 wies einen Winkel von $0,1^{\circ}( \pm 5,9)$ auf. G3 und G4 präsentierten einen AI von $-0,9^{\circ}( \pm 5,7)$ bzw. $-0,7^{\circ}( \pm 5,4)$. Es ergab sich ein signifikanter $\mathrm{p}$-Wert $(<0,0001)$.

AcetAVa: Bei der AcetAVa konnten signifikante Unterschiede zwischen den Altersgruppen nachgewiesen werden $(\mathrm{p}<0,0001)$. Des Weiteren zeigte sich eine mit dem Alter ansteigende Winkelgröße (G1 18,5 $[ \pm 5,9]$, G2 20,4 $[ \pm 6,5]$, G3 20,9 $[ \pm 7,0]$, G4 23, $\left.3^{\circ}[ \pm 6,5]\right)$.

AASA: Der AASA präsentierte einen Wert von $63,4^{\circ}( \pm 8,8)$ in G1. G2 und G3 wiesen zur jeweils vorhergehenden Gruppe größere Winkel auf $\left(65,6^{\circ}[ \pm 8,8]\right.$ und $\left.67,1^{\circ}[ \pm 9,2]\right)$. Der AASA von G4 $\left(65,7^{\circ}[ \pm 10,2]\right)$ war jedoch kleiner als der von G3. Die Auswertung ergab signifikante altersabhängige Unterschiede ( $\mathrm{p}$-Wert<0,0001).

PASA: Mit aufsteigenden Altersgruppen stieg der PASA stetig an, beginnend bei $100,0^{\circ}$ $( \pm 11,2)$ in $\mathrm{G} 1$, über $106,0^{\circ}( \pm 12,1)$ in $\mathrm{G} 2$ und $108,7^{\circ}( \pm 12,8)$ in $\mathrm{G} 3$, bis hin zu $112,6^{\circ}( \pm 13,7)$ in G4. Der p-Wert erbrachte ein signifikantes Ergebnis $(<0,0001)$. 
CCD: Invers zu dem beim PASA dargestellten Anwachsen der Winkelgröße, nahm der CCD mit zunehmendem Alter ab. In G1 und konnte ein Winkel von $131,4^{\circ}$ festgestellt werden und in G2 einer von $131,3^{\circ}$. Die Standardabweichungen betrugen $\pm 8,5$ in G1 sowie $\pm 7,9$ in G2. In G3 sowie G4 wurden Werte von $129,4^{\circ}( \pm 8,3)$ und $128,9^{\circ}( \pm 9,3)$ gemessen. Die Unterschiede zwischen den Altersgruppen waren signifikant (p-Wert<0,0001).

SaA: Ebenso wie beim CCD zeigte sich für den $\mathrm{S} \alpha \mathrm{A}$ eine mit zunehmendem Alter abnehmende Gradzahl (G1 47, $\left.0^{\circ}[ \pm 8,2], \mathrm{G} 246,0^{\circ}[ \pm 8,3], \mathrm{G} 345,7^{\circ}[ \pm 8,5], \mathrm{G} 446,2^{\circ}[ \pm 9,1]\right)$, wobei der p-Wert nicht signifikant war $(0,21)$.

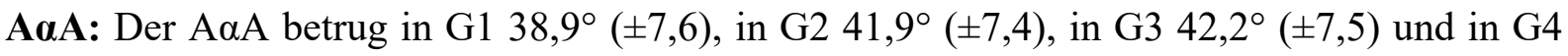
$39,3^{\circ}( \pm 7,7)$. Es konnten signifikante Altersunterschiede festgestellt werden $(p<0,0001)$.

FNV: Bei der FNV wurden in G1 $11,4^{\circ}( \pm 11,5)$ und in G2 $11,8^{\circ}( \pm 10,2)$ gemessen. In den höheren Altersgruppen (G3 und G4) fanden sich Werte von $11,7^{\circ}( \pm 9,6)$ sowie $12,3^{\circ}( \pm 8,2)$. Die Unterschiede zwischen den Gruppen waren nicht signifikant $(\mathrm{p}=0,85)$.

SW: Die Mittelwerte des SW nahmen mit ansteigenden Altersgruppen ab. Diese betrugen 40,2 $( \pm 4,3), 37,0^{\circ}( \pm 4,4), 36,3^{\circ}( \pm 4,5)$ und $35,5^{\circ}( \pm 5,2)(\mathrm{G} 1-4)$. Es konnte ein signifikanter p-Wert festgestellt werden $(\mathrm{p}<0,0001)$.

Nachfolgend werden die Ergebnisse der Offset-Parameter sowie des Femurkopf-/ Pfannendurchmessers und des Verhältnisses von Femurkopf- zum Pfannendurchmesser dargestellt. Alle Parameter zeigten signifikante Unterschiede zwischen den Altersgruppen.

FO: Das FO zeigte Werte von $37,6 \mathrm{~mm}( \pm 7.5)$ in $\mathrm{G} 1$ und $39,0 \mathrm{~mm}( \pm 8,0) \mathrm{mm}$ in G2. Die Mittelwerte in G3 bzw. G4 betrugen 41,2 mm $( \pm 8,1)$ sowie $41,6 \mathrm{~mm}( \pm 8,1)$. Es ergaben sich signifikante Messunterschiede $(\mathrm{p}<0,0001)$.

AO: Für das AO wurden ansteigende Mittelwerte in den Altersgruppen G1 bis G3 festgestellt $(31,2 \mathrm{~mm}[ \pm 3,7], 32,0 \mathrm{~mm}[ \pm 4,5]$ und 32,3 $\mathrm{mm}[ \pm 4,6])$. G4 präsentierte ein kleineres Ergebnis als G3 $(30,4 \mathrm{~mm}[ \pm 4,5])$. Die Messunterschiede zwischen den Gruppen waren signifikant $(\mathrm{p}<0,0001)$.

GO: Das GO betrug in G1 68,7 mm $( \pm 8,7)$ und in G2 71,0 mm $( \pm 9,7)$. In G3 bzw. G4 zeigten sich Mittelwerte von 73,5 mm $( \pm 9,7)$ sowie 71,9 mm $( \pm 9,3)$. Die Auswertungen erbrachten einen signifikanten $\mathrm{p}$-Wert $(<0,0001)$. 
ØF: Der Femurkopfdurchmesser zeigte in G1 einen Mittelwert von 46,2 mm $( \pm 3,6)$. In G2 betrug der Mittelwert 47,3 mm $( \pm 4,1)$, in G3 48,4 $\mathrm{mm}( \pm 4,1)$ und in G4 47,3 $\mathrm{mm}( \pm 4,0)$. Die altersabhängigen Messunterschiede waren signifikant $(\mathrm{p}<0,0001)$.

ØA: Die Analyse ergab im Mittel einen Durchmesser des Acetabulums von 58,1 mm $( \pm 4,6)$ in G1. Die anderen Altersgruppen (G2-4) präsentierten folgende Ergebnisse: 57,8 mm $( \pm 5,3)$, $59,1 \mathrm{~mm}( \pm 5,4)$ und 57,9 $\mathrm{mm}( \pm 5,7)$. Der $\mathrm{p}$-Wert war signifikant $(0,0006)$.

F/A: Das Verhältnis von Femurkopf- zur Acetabulumdurchmesser unterschied sich nur in G1 signifikant $(p<0,0001)$ von den anderen drei Altersgruppen und betrug 79,6\% $( \pm 0,0)$. In G2, G3 sowie G4 ergaben sich Mittelwerte von $82,0 \%( \pm 0,1)$.

Die vier Altersgruppen unterschieden sich signifikant ( $p$-Wert $<0,0001$ ) für den gemessenen mittleren Arthrosegrad. In der Folge wird für jeden Arthrosegrad (KL: 0-4) einzeln die Anzahl der Hüftgelenke dargestellt, die in den vier Altersgruppen dem jeweiligen Arthrosegrad zugeordnet wurden. In Prozent wird angegeben, wie viele Hüften in einer Altersgruppe einem bestimmten Arthrosegrad zugehörig sind. Diese Auswertung erfolgt für das Gesamtkollektiv $(n=1424)$ und wird nicht nach der der Hüftseite (rechts bzw. links) unterschieden.

Grad 0: Keine Arthrose zeigten 96 Hüften (37,2\%) in G1, 37 Hüften (10,0\%) in G2 und 56 Hüften $(8,4 \%)$ in G3. In G4 wurde keine Hüfte mit einem KL 0 gefunden.

Grad 1: In der Altersgruppe 19-40 Lebensjahre fanden sich 93 (36,1\%) Hüften mit einem KL von 1. 139 Hüftgelenke (37,6\%) bei den 40-60-jährigen und 210 (31,4\%) Hüftgelenke bei den 60-80-jährigen zeigten eine KL 1. In der Altersgruppe >80 Jahre hatten 30 (23,4\%) Hüftgelenke einen Arthrosegrad 1.

Grad 2: Hier ergab sich folgende Verteilung: G1: 69 (26,7\%) Hüftgelenke, G2: 182 (49,2\%) Hüftgelenke, G3: 353 (52,8\%) Hüftgelenke und G4: 69 (53,9\%) Hüftgelenke.

Grad 3: In G1 wurde kein Hüftgelenk mit einem KL 3 gefunden. In G2 wurden 9 (2,4\%) und in G3 44 (6,6\%) Hüftgelenke mit einem Arthrosegrad 3 bewertet. $24(18,8 \%)$ Hüftgelenke mit einem entsprechenden Arthrosegrad wurden in G4 gefunden.

Grad 4: In der Altersgruppe 19-40 Jahre wurden keine Hüftgelenke mit den radiologischen Zeichen einer fortgeschrittenen Arthrose gefunden. In den Altersgruppen G2 bzw. G3 zeigten sich $3(0,8 \%, G 2)$ bzw. $5(0,8 \%, G 3)$ Hüftgelenke, die mit einem KL von 4 klassifiziert werden konnten. In G4 fanden sich 5 (3,9\%) Hüftgelenke. 
Nachfolgend werden sämtliche Ergebnisse grafisch in den Abbildungen 14, 15, 16 und 17 veranschaulicht.

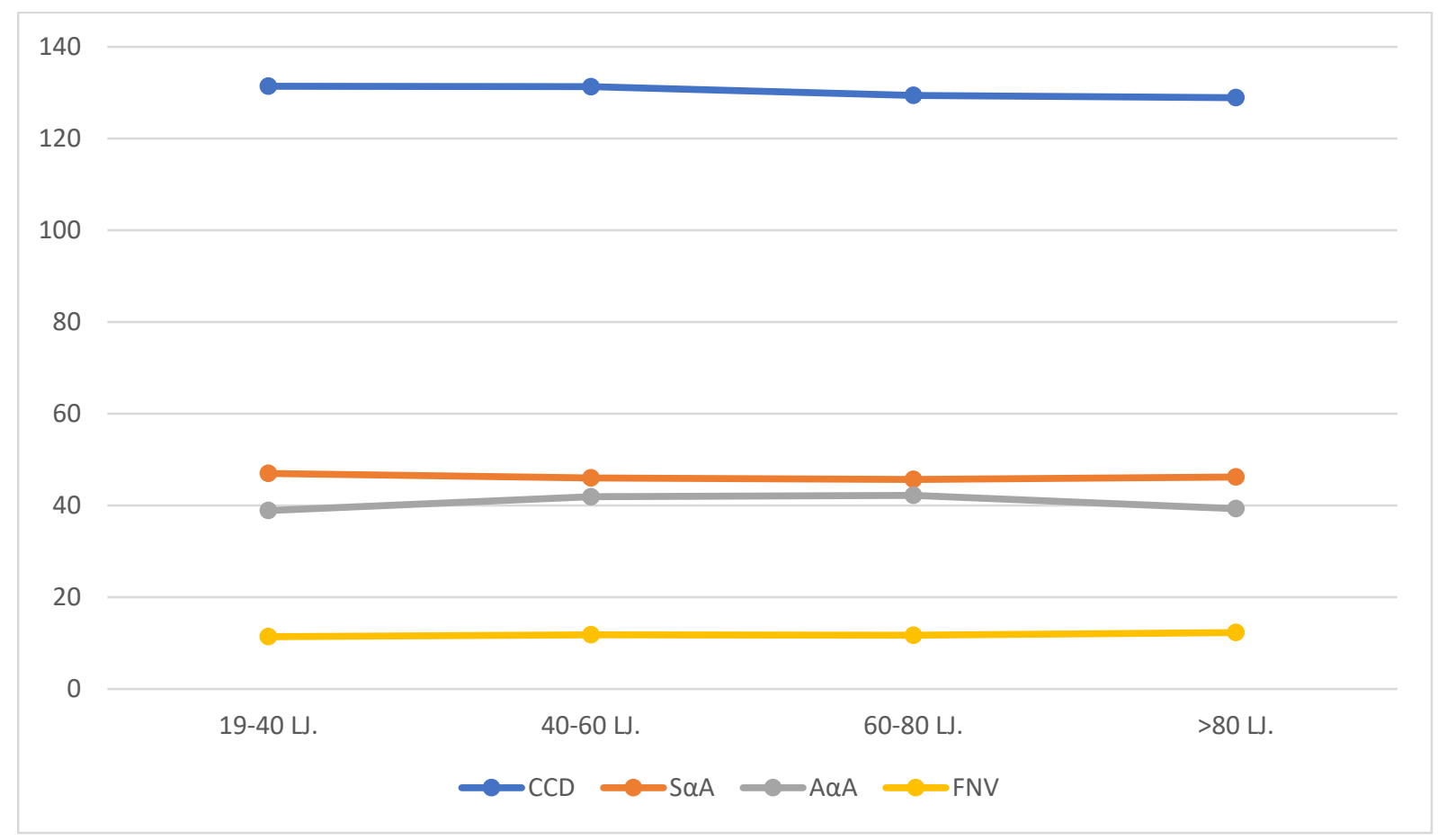

Abb. 14 Grafische Darstellung der Mittelwerte in Winkelgrad nach Altersgruppen unterteilt für die femoralen Messparameter

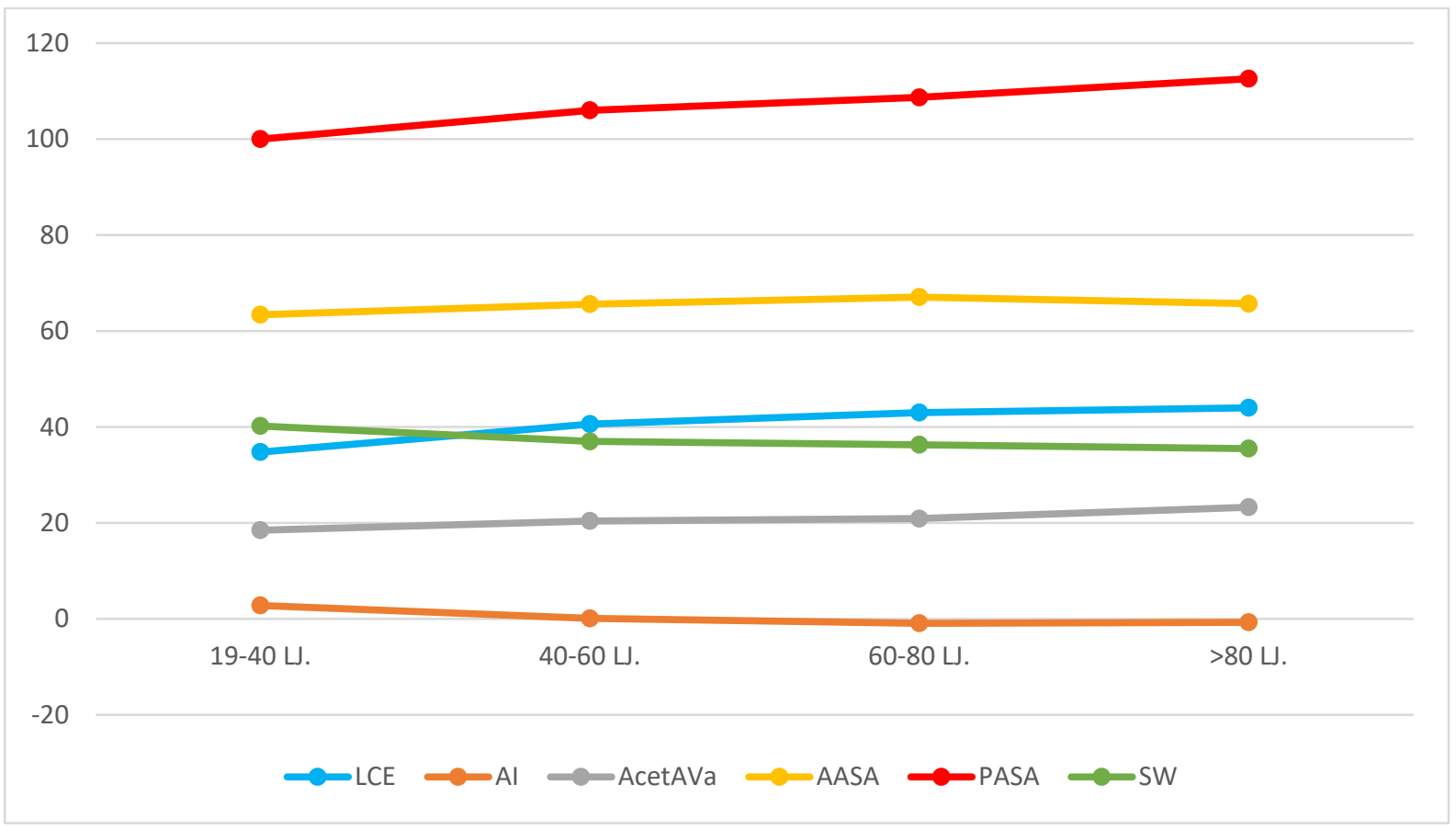

Abb. 15 Grafische Darstellung der Mittelwerte in Winkelgrad nach Altersgruppen unterteilt für die acetabulären Messparameter 


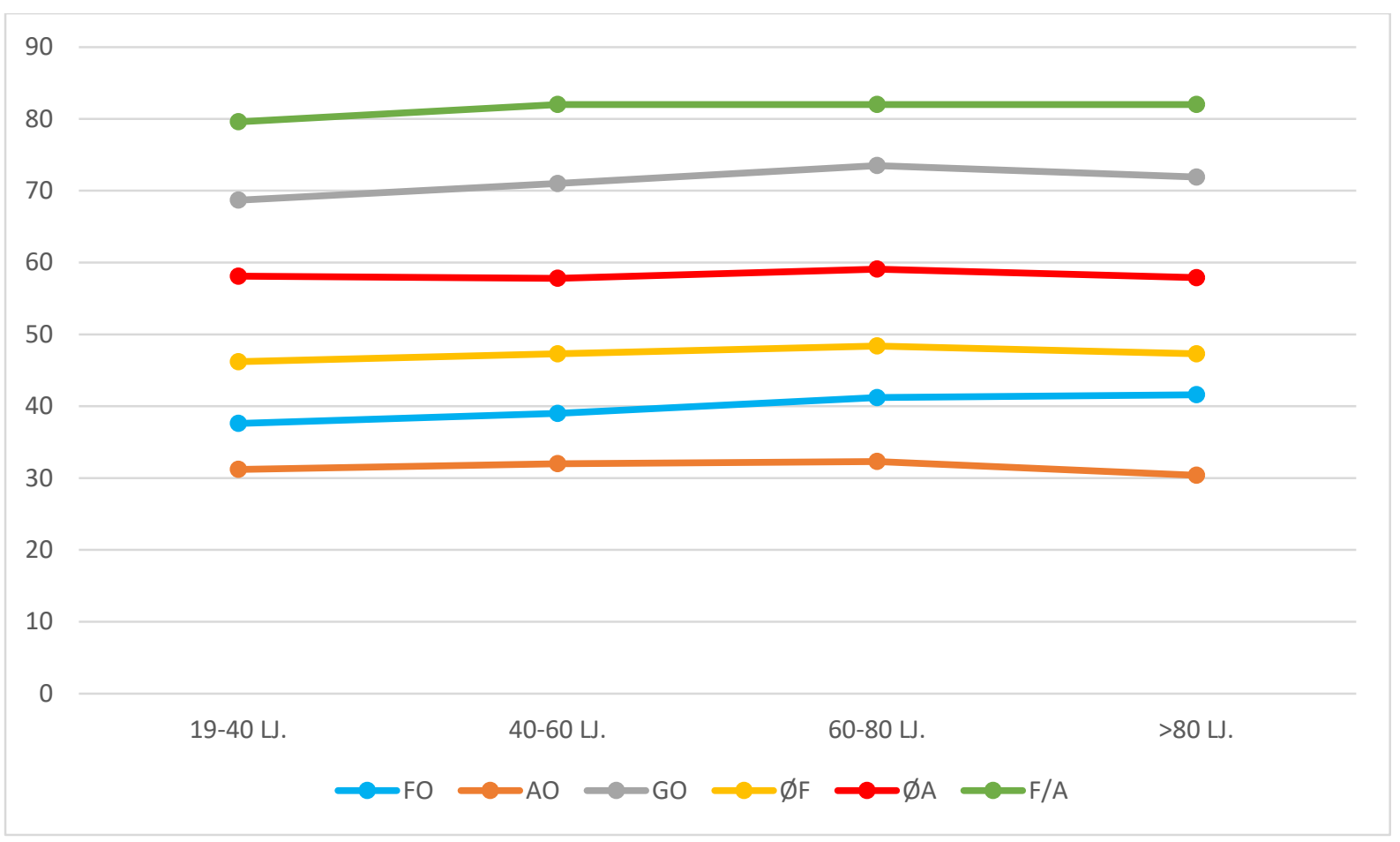

Abb. 16 Grafische Darstellung der Mittelwerte nach Altersgruppen unterteilt für die Offset-Parameter sowie Femurkopf- und Acetabulumdurchmesser in $\mathrm{mm}$ und das Verhältnis F/A in \%

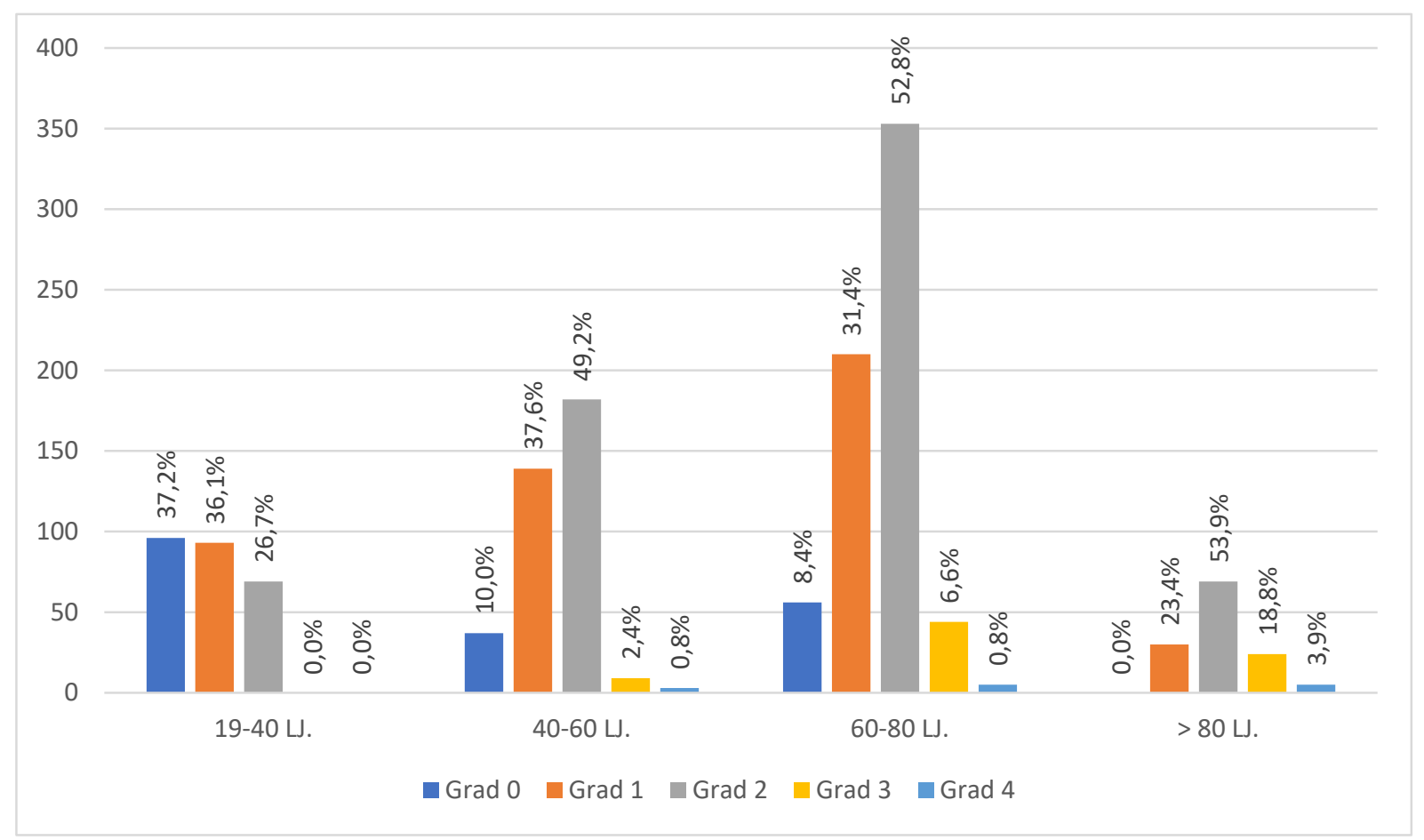

Abb. 17 Grafische Darstellung der Verteilung des Arthrosegrades nach Kellgren/Lawrence in den Altersgruppen G1-G4 
In allen vier Abbildungen sind auf der X-Achse die Altersgruppen abgebildet (Abb. 14, 15, 16 und 17). Die Skalierung der y-Achse gibt absolute Zahlwerte ohne Einheit wieder, sodass daran sowohl die Einheit Winkelgrad, als auch der Durchmesser in mm sowie das Verhältnis in Prozent abgelesen werden können (Abb. 14, 15 und 16). Aus Übersichtsgründen wurden FO, ØF (auch femorale Messparameter), AO und ØA (auch acetabuläre Messparameter) in Abb. 16 integriert.

Die absoluten Werte der y-Achse in Abb. 17 beziehen sich auf die Anzahl an betroffenen Hüftgelenken.

\subsubsection{Geschlechtsspezifische Unterschiede}

Weiterhin wurden geschlechtsspezifische Unterschiede für die gemessenen Parameter untersucht. Die Auseinandersetzung mit diesem Aspekt ist die Umsetzung der vierten Fragestellung der vorliegenden Arbeit. Tabelle 10 und 11 zeigen die Messdatenverteilung.

Tab. 10 Geschlechtsspezifische Verteilung der femoralen und acetabulären Parametern

\begin{tabular}{|c|c|c|c|c|c|}
\hline & & $\begin{array}{l}\text { gesamt } \\
n=1424\end{array}$ & $\begin{array}{l}\text { Männer } \\
\text { n=942 }\end{array}$ & $\begin{array}{l}\text { Frauen } \\
n=482\end{array}$ & p-Werte \\
\hline $\mathrm{LCE}$ & {$\left[\mathrm{in}^{\circ}\right]$} & $40,9( \pm 8,6)$ & $40,5( \pm 8,2)$ & $41,8( \pm 9,4)$ & 0,02 \\
\hline AI & {$\left[\mathrm{in}^{\circ}\right]$} & $0,1( \pm 5,8)$ & $-0,1( \pm 5,6)$ & $0,4( \pm 6,2)$ & 0,12 \\
\hline AcetAVa & {$\left[\right.$ in $\left.^{\circ}\right]$} & $20,5( \pm 6,7)$ & $19,2( \pm 6,3)$ & $23,1( \pm 6,8)$ & $<0,0001$ \\
\hline AASA & {$\left[\mathrm{in}^{\circ}\right]$} & $65,9( \pm 9,2)$ & $66,5( \pm 8,9)$ & $64,9( \pm 9,7)$ & 0,002 \\
\hline PASA & {$\left[\mathrm{in}^{\circ}\right]$} & $106,8( \pm 12,9)$ & $104,8( \pm 12,0)$ & $110,7( \pm 13,8)$ & $<0,0001$ \\
\hline $\mathrm{CCD}$ & {$\left[\mathrm{in}^{\circ}\right]$} & $130,2( \pm 8,4)$ & $130,6( \pm 8,4)$ & $129,4( \pm 8,3)$ & 0,014 \\
\hline $\mathrm{S} \alpha \mathrm{A}$ & {$\left[\mathrm{in}^{\circ}\right]$} & $46,1( \pm 8,5)$ & $46,3( \pm 8,1)$ & $45,6( \pm 9,1)$ & 0,15 \\
\hline $\mathrm{A} \alpha \mathrm{A}$ & {$\left[\mathrm{in}^{\circ}\right]$} & $41,2( \pm 7,6)$ & $42,1( \pm 7,7)$ & $39,6( \pm 7,1)$ & $<0,0001$ \\
\hline FNV & {$\left[\mathrm{in}^{\circ}\right]$} & $11,7( \pm 10,0)$ & $10,5( \pm 9,7)$ & $14,1( \pm 10,2)$ & $<0,0001$ \\
\hline SW & {$\left[\mathrm{in}^{\circ}\right]$} & $37,1( \pm 4,8)$ & $36,8( \pm 4,5)$ & $37,6( \pm 5,2)$ & 0,004 \\
\hline FO & [in $\mathrm{mm}]$ & $40,0( \pm 8,1)$ & $40,8( \pm 8,3)$ & $38,4( \pm 7,3)$ & $<0,0001$ \\
\hline $\mathrm{AO}$ & [in $\mathrm{mm}$ ] & $31,8( \pm 4,5)$ & $33,7( \pm 3,6)$ & $28,2( \pm 3,6)$ & $<0,0001$ \\
\hline GO & [in $\mathrm{mm}]$ & $71,8( \pm 9,7)$ & $74,5( \pm 9,1)$ & $66,6( \pm 8,5)$ & $<0,0001$ \\
\hline$\varnothing \mathrm{F}$ & [in $\mathrm{mm}]$ & $47,6( \pm 4,1)$ & $49,4( \pm 3,4)$ & $44,2( \pm 2,9)$ & $<0,0001$ \\
\hline ØА & [in $\mathrm{mm}$ ] & $58,5( \pm 5,3)$ & $60,6( \pm 4,5)$ & $54,2( \pm 3,9)$ & $<0,0001$ \\
\hline F/A & [in \%] & $81,6( \pm 0,1)$ & $81,6( \pm 0,1)$ & $81,6( \pm 0,1)$ & 0,80 \\
\hline
\end{tabular}


Tab. 11 Geschlechtsspezifische Verteilung der Arthrosewerte (nach Kellgren/Lawrence)

\begin{tabular}{|c|c|c|c|c|}
\hline & $\begin{array}{c}\text { gesamt } \\
\mathbf{n = 1 4 2 4}\end{array}$ & $\begin{array}{c}\text { Männer } \\
\mathbf{n = 9 4 2}\end{array}$ & $\begin{array}{c}\text { Frauen } \\
\mathbf{n = 4 8 2}\end{array}$ & p-Werte \\
\hline Grad & & & & 0,034 \\
0 & $189(13,3 \%)$ & $125(13,3 \%)$ & $64(13,3 \%)$ & \\
1 & $472(33,2 \%)$ & $327(34,7 \%)$ & $145(30,1 \%)$ & \\
2 & $673(47,3 \%)$ & $428(45,4 \%)$ & $245(50,8 \%)$ & \\
3 & $77(5,4 \%)$ & $57(6,1 \%)$ & $20(4,2 \%)$ & \\
4 & $13(0,9 \%)$ & $5(0,5 \%)$ & $8(1,7 \%)$ & \\
\hline
\end{tabular}

Von den 1424 vermessenen Hüften waren 942 männliche (471 Patienten) und 482 weibliche (241 Patientinnen) Hüften. Die Bestimmung der Mittelwerte mit den dazugehörigen Standardabweichungen sowie der Erhebung der p-Werte ergab für die femoralen und acetabulären Parameter folgende Verteilung der Messwerte:

LCE: Der mittlere LCE zeigte in den männlichen $40,5^{\circ}( \pm 8,2)$ sowie $41,8^{\circ}( \pm 9,4)$ in den weiblichen Hüftgelenken. Es ergaben sich signifikante Messunterschiede $(p=0,02)$.

AI: Die untersuchten Männer wiesen einen Mittelwert von $-0,1^{\circ}( \pm 5,6)$ für den AI auf und die Frauen einen mittleren AI von $0,4^{\circ}( \pm 6,2)$. Dieser Unterschied war nicht signifikant $(p=0,12)$.

AcetAVa: Die AcetAVa präsentierte sich für die maskulinen Gelenke mit $19,2^{\circ}( \pm 6,3)$ kleiner als für die femininen mit $23,1^{\circ}( \pm 6,8)$. Der Messunterschied war signifikant $(\mathrm{p}<0,0001)$.

AASA: Die Analyse ergab einen signifikanten Unterschied $(\mathrm{p}=0,002)$ von $1,6^{\circ}$ für den AASA zwischen den Männern $\left(66,5^{\circ}[ \pm 8,9]\right)$ und den Frauen $\left(64,9^{\circ}[ \pm 9,7]\right)$.

PASA: In männlichen Hüftgelenken betrug der PASA $104,8^{\circ}( \pm 12,0)$. Die weiblichen Gelenke wiesen im Mittel mit 110,7 $( \pm 13,8)$ einen Winkel auf, der 5,9 über dem der Männer lag. Es wurde ein $\mathrm{p}$-Wert $<0,0001$ ermittelt.

CCD: Der CCD unterschied sich zwischen den Männern $\left(130,6^{\circ}[ \pm 8,4]\right)$ und den Frauen $\left(129,4^{\circ}[ \pm 8,3]\right)$ nur gering, jedoch signifikant $(p=0,014)$.

SaA: Die Analyse ergab im Mittel einen S $\alpha$ A von $46,3^{\circ}( \pm 8,1)$ in den männlichen Hüften und von $45,6^{\circ}( \pm 9,1)$ in den weiblichen Hüften. Der p-Wert war nicht signifikant $(0,15)$.

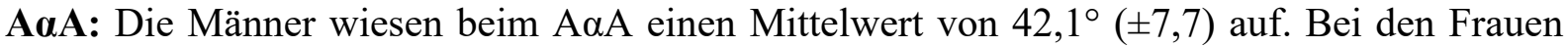
wurde ein Winkel von $39,6^{\circ}( \pm 7,1)$ gemessen. Der Messunterschied war signifikant $(\mathrm{p}<0,0001)$. 
FNV: Bei der FNV fanden sich folgende Ergebnisse: $10,5^{\circ}( \pm 9,7)$ in den männlichen sowie $14,1^{\circ}( \pm 10,2)$ in den weiblichen Gelenken. Die Auswertungen ergaben einen signifikanten p-Wert $(<0,0001)$.

SW: Maskuline Hüften zeigten einen SW von $36,8^{\circ}( \pm 4,5)$, feminine einen SW von $37,6^{\circ}$ $( \pm 5,2)$. Der geschlechtsspezifische Unterschied war signifikant $(p=0,004)$.

Alle Offset-Parameter sowie die Durchmesser des Femurkopfes und des Acetabulums wiesen im Mittel größere Werte bei den untersuchten Männern als bei den untersuchten Frauen auf. Sämtliche Parameter zeigten signifikante Messunterschiede.

FO: Bei den maskulinen zeigte sich im Mittel ein 2,4 mm größeres FO als bei den femininen Gelenken (40,8 mm ( $( \pm 8,3)$ im Unterschied zu 38,4 mm ( $( \pm 7,3))$. Die Auswertung erbrachte einen signifikanten $\mathrm{p}-\mathrm{Wert}(<0,0001)$.

AO: Für das AO konnten Werte von 33,7 mm $( \pm 3,6)$ bei den Männern und 28,2 $\mathrm{mm}( \pm 3,6)$ bei den Frauen gemessen werden. Der p-Wert war signifikant $(<0,0001)$.

GO: Das GO präsentierte einen signifikanten geschlechtsspezifischen Unterschied $(p<0,0001)$ von 7,9 mm $(74,5 \mathrm{~mm}[ \pm 9,1]$ gemessen in den männlichen Hüften sowie $66,6 \mathrm{~mm}[ \pm 8,5]$ in den weiblichen).

ØF: Der männliche Femurkopfdurchmesser ergab im Mittel 49,4 mm $( \pm 3,4)$, der weibliche 44,2 mm ( $\pm 2,9)$. Der Messunterschied war signifikant ( $\mathrm{p}$-Wert<0,0001).

ØA: Beim Pfannendurchmesser betrug der signifikante geschlechtsspezifische Unterschied $(\mathrm{p}<0,0001)$ 6,4 mm (Männer 60,6 mm $( \pm 4,5)$, Frauen 54,2 mm $( \pm 3,9))$.

F/A: Die Analyse ergab für beide Geschlechter im Mittel einen Wert von $81,6 \%( \pm 0,1)$ für das Verhältnis aus Femurkopf- und Acetabulumdurchmesser. Der p-Wert war nicht signifikant und betrug 0,80 .

Die Ergebnisse der vorliegenden Arthrosestadien in Hinsicht auf geschlechtsspezifische Unterschiede zeigten signifikante Unterschiede $(\mathrm{p}=0,034)$. Die absoluten Wertangaben und die Prozentangaben beziehen sich auf die Anzahl der männlichen (942) und der weiblichen (482) vermessenen Hüften.

Grad 0: Keine Anzeichen für Arthrose wurden in 125 (13,3\%) maskulinen Gelenken und in 64 $(13,3 \%)$ femininen gefunden. 
Grad 1: Von den untersuchten Männern ließen sich 327 (34,7\%) Hüften in Grad 1 nach Kellgren/Lawrence einteilen. Bei den Frauen waren es 145 (30,1\%).

Grad 2: 428 (45,4\%) männliche sowie 245 (50,8\%) weibliche Hüftgelenke zeigten einen KL von 2 .

Grad 3: In $57(6,1 \%)$ maskulinen Hüftgelenken und in $20(4,2 \%)$ femininen Hüftgelenken wurde ein Arthrosegrad 3 gemessen.

Grad 4: Das Vollbild einer radiologischen Arthrose (KL 4) zeigte sich bei den Männern in 5 $(0,5 \%)$ und bei den Frauen in $8(1,7 \%)$ Hüftgelenken.

\subsubsection{Einteilung des Kollektivs nach pathologischen Werten der Referenzliteratur}

In der Einleitung wurden die durch die Literatur als pathologisch definierten Referenzwerte zum Teil bereits dargestellt. Unter 2.2 wurde auf diese ausführlicher eingegangen.

Unter Berücksichtigung von diesen Angaben in der statistischen Auswertung ergaben sich die in Tabelle 12 dargestellten Werte.

Tab. 12 Anzahl der Hüftgelenke mit pathologischen Parametern

\begin{tabular}{|c|c|c|c|c|c|}
\hline Parameter & & $\begin{array}{c}\text { links } \\
\text { absolut }\end{array}$ & $\begin{array}{c}\text { links } \\
\mathbf{\%}\end{array}$ & $\begin{array}{c}\text { rechts } \\
\text { absolut }\end{array}$ & $\begin{array}{c}\text { rechts } \\
\mathbf{\%}\end{array}$ \\
\hline $\mathrm{CCD}<125^{\circ}$ & Coxa vara & 124 & $17,42 \%$ & $\mathbf{2 3 5}$ & $\mathbf{3 3 , 0 1 \%}$ \\
$\mathrm{CCD}>140^{\circ}$ & Coxa valga & 99 & $13,90 \%$ & 54 & $7,58 \%$ \\
$\mathrm{FNV}<0^{\circ}$ & & 66 & $9,27 \%$ & 83 & $11,66 \%$ \\
$\mathrm{FNV}<5^{\circ}$ & Coxa retrotorta & $\mathbf{1 7 7}$ & $\mathbf{2 4 , 8 6 \%}$ & $\mathbf{1 8 5}$ & $\mathbf{2 5 , 9 8 \%}$ \\
$\mathrm{FNV}>25^{\circ}$ & Coxa antetorta & 61 & $8,57 \%$ & 51 & $7,16 \%$ \\
$\mathrm{~A} \alpha \mathrm{A}>55^{\circ}$ & Cam-Impingement & 10 & $1,40 \%$ & 36 & $5,06 \%$ \\
$\mathrm{LCE}<20^{\circ}$ & Dysplasie & 0 & $0,00 \%$ & 5 & $0,70 \%$ \\
$\mathrm{LCE} 20-25^{\circ}$ & Borderline & 5 & $0,70 \%$ & 24 & $3,37 \%$ \\
$\mathrm{LCE}>40^{\circ}$ & Overcoverage (Pincer) & $\mathbf{4 3 9}$ & $\mathbf{6 1 , 6 6 \%}$ & $\mathbf{2 9 9}$ & $\mathbf{4 1 , 9 9 \%}$ \\
$\mathrm{AcetAVa}<0^{\circ}$ & & 0 & $0,00 \%$ & 0 & $0,00 \%$ \\
$\mathrm{AcetA} \mathrm{Va}>15^{\circ}$ & & $\mathbf{5 6 3}$ & $\mathbf{7 9 , 0 7 \%}$ & $\mathbf{5 6 4}$ & $\mathbf{7 9 , 2 1 \%}$ \\
$\mathrm{AASA}<52^{\circ}$ & Dysplasie & 53 & $7,44 \%$ & 31 & $4,35 \%$ \\
$\mathrm{PASA}<90^{\circ}$ & Dysplasie & $\mathbf{7 7}$ & $10,81 \%$ & 52 & $7,30 \%$ \\
$\mathrm{SW}<34^{\circ}$ & Overcoverage schwer & $\mathbf{1 9 8}$ & $\mathbf{2 7 , 8 1 \%}$ & $\mathbf{1 5 9}$ & $\mathbf{2 2 , 3 3 \%}$ \\
$\mathrm{SW} 34-37,9^{\circ}$ & Overcoverage & $\mathbf{1 9 9}$ & $\mathbf{2 7 , 9 5 \%}$ & $\mathbf{2 3 8}$ & $\mathbf{3 3 , 4 3 \%}$ \\
$\mathrm{SW}>43^{\circ}$ & Dysplasie & 55 & $7,72 \%$ & 92 & $12,92 \%$ \\
\hline
\end{tabular}

(Referenzwerte in der Literatur; rot markiert = Anhäufung pathologischer Werte laut Literatur) 
Die prozentualen Angaben beziehen sich auf die jeweilige Hüftseite (712 rechte und 712 linke Hüften). Farblich wurden die Werte hervorgehoben, die in über 20\% der Hüftgelenke laut Vergleichsliteratur als pathologisch zu beurteilen wären. Die folgenden Ausführungen legen die in der Einleitung dargestellten Aussagen der Vergleichsstudien als Referenzen zu Grunde. Aussagen bezüglich möglicher Pathologien gehen nur auf diese Referenzwerte zurück. Im unmittelbaren weiteren Verlauf erfolgt keine erneute Nennung der genauen Literaturquelle des jeweiligen Referenzwertes. Eine Coxa vara $\left(\mathrm{CCD}<125^{\circ}\right)$ wurde in 235 rechten Hüften (33,01\%) und in 124 linken Hüften festgestellt (17,42\%). Im Gegensatz dazu trat eine Coxa valga $\left(\mathrm{CCD}>140^{\circ}\right)$ vermehrt linksseitig auf (99 zu 54 bzw. 13,90\% zu 7,58\%). Die Verhältnisse betrugen für beide Referenzbereiche jeweils annährend 2:1. Physiologische Werte (CCD: $\left.125-140^{\circ}\right)$ fanden sich in 489 linken Hüften $(68,68 \%)$ und in 423 rechten Hüften $(59,41 \%)$.

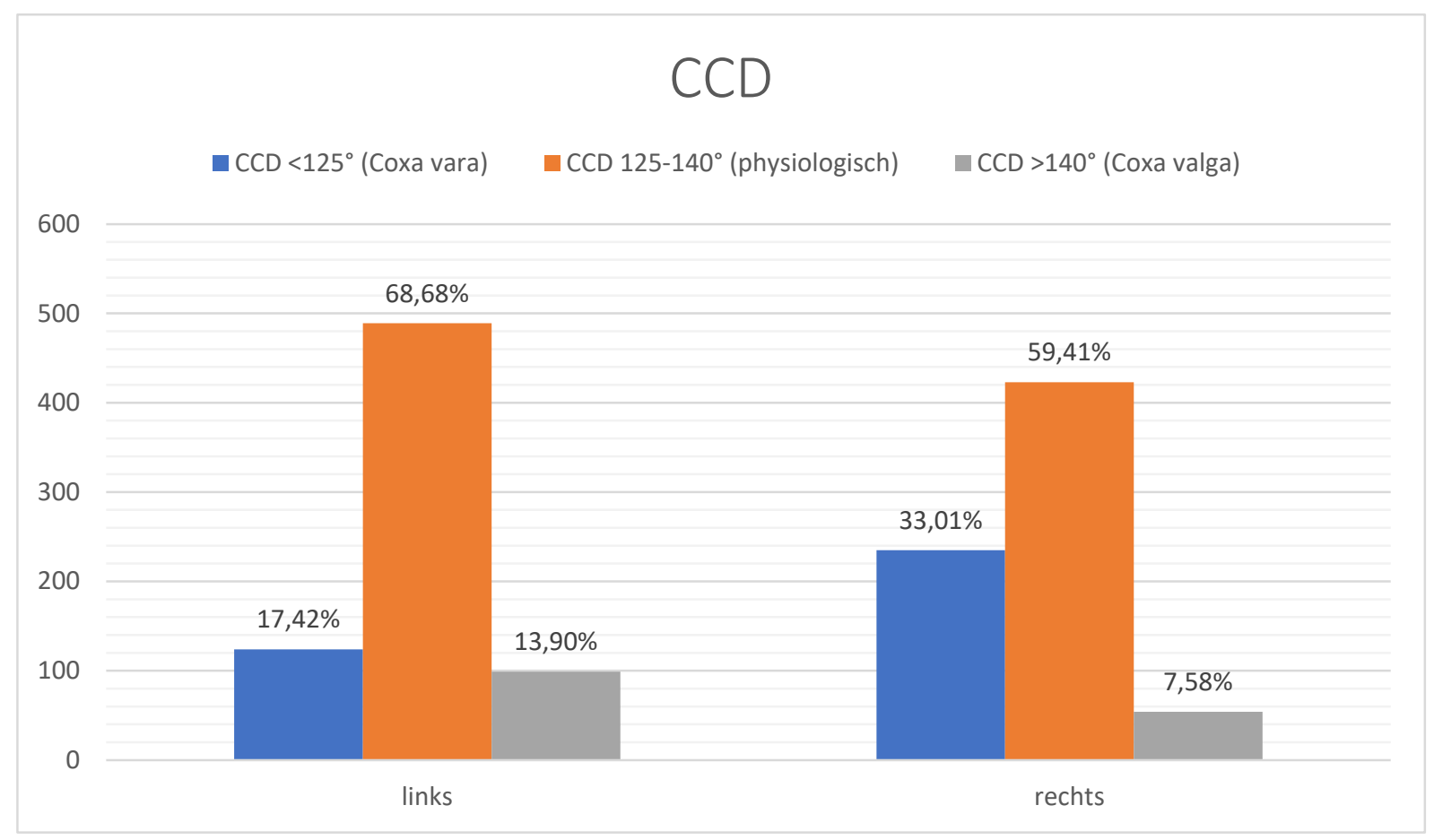

Abb. 18 Verteilung der physiologischen und pathologischen CCD-Werte im Gesamtkollektiv nach Seite unterteilt

Im untersuchten Patientenkollektiv fanden sich 177 Hüftgelenke (24,86\%) auf der linken und 185 (25,98\%) auf der rechten Seite, die die Kriterien einer Coxa retrotorta (FNV $\left.<5^{\circ}\right)$ erfüllten. 
Eine manifeste Retroversion (FNV $<0^{\circ}$ ) wurde in $66(9,27 \%$ ) linken respektive 83 rechten Hüftgelenken (11,66\%) gefunden. Ähnlich präsentierte sich die Ergebnisverteilung für die Auswertung hinsichtlich einer Coxa antetorta $\left(\mathrm{FNV}>25^{\circ}\right)$. Im Vergleich fanden sich 61 (8,57\%) linke Hüften und 51 (7,16\%) rechte Hüften, die radiologisch eine solche Pathologie aufwiesen. Eine physiologische FNV (5-25) wurde in 408 (57,30\%) linken Hüften und in 393 $(55,20 \%)$ rechten Hüften gefunden. Abbildung 19 zeigt die Verteilung grafisch.

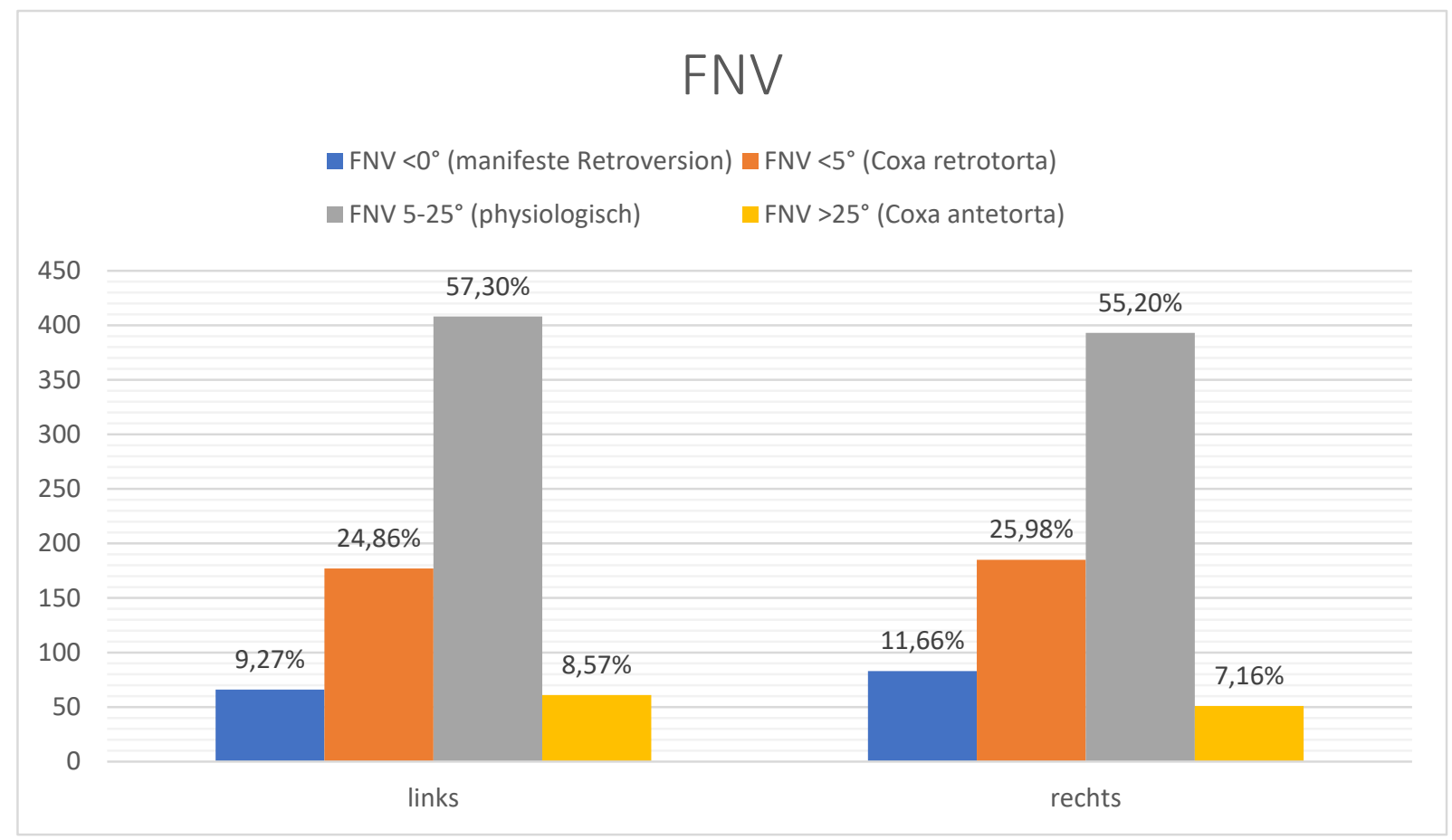

Abb. 19 Verteilung der physiologischen und pathologischen Femoral neck version im Gesamtkollektiv nach Seite unterteilt

Der $\mathrm{A} \alpha \mathrm{A}$ in seiner Funktion als möglicher prädiktiver Marker für ein Cam-Impingment mit einem Wert $>55^{\circ}$ wurde in dem vorliegenden Kollektiv in $36(5,06 \%)$ rechten und in $10(1,40 \%)$ linken Hüftgelenken gefunden.

Ein LCE $>40^{\circ}$, als möglicher Indikator für ein Pincer-Impingment, wurde in 299 (41,99\%) rechten und in $439(61,66 \%)$ linken Hüftgelenken gemessen. Eine Dysplasie (LCE <20 $)$ wurden nur in 5 rechten Hüften $(0,70 \%)$ und in keiner der linken Hüften gefunden. 24 rechte $(3,37 \%)$ und 5 linke Hüftegelenke $(0,70 \%)$ zeigten eine Borderline-Konfiguration (LCE: $\left.20-25^{\circ}\right)$. Physiologische Werte $\left(25,1-40^{\circ}\right)$ wurden in $268(37,64 \%)$ linken Hüften und in 384 $(53,93 \%)$ rechten Hüften gefunden. Abbildung 20 veranschaulicht dieses. 


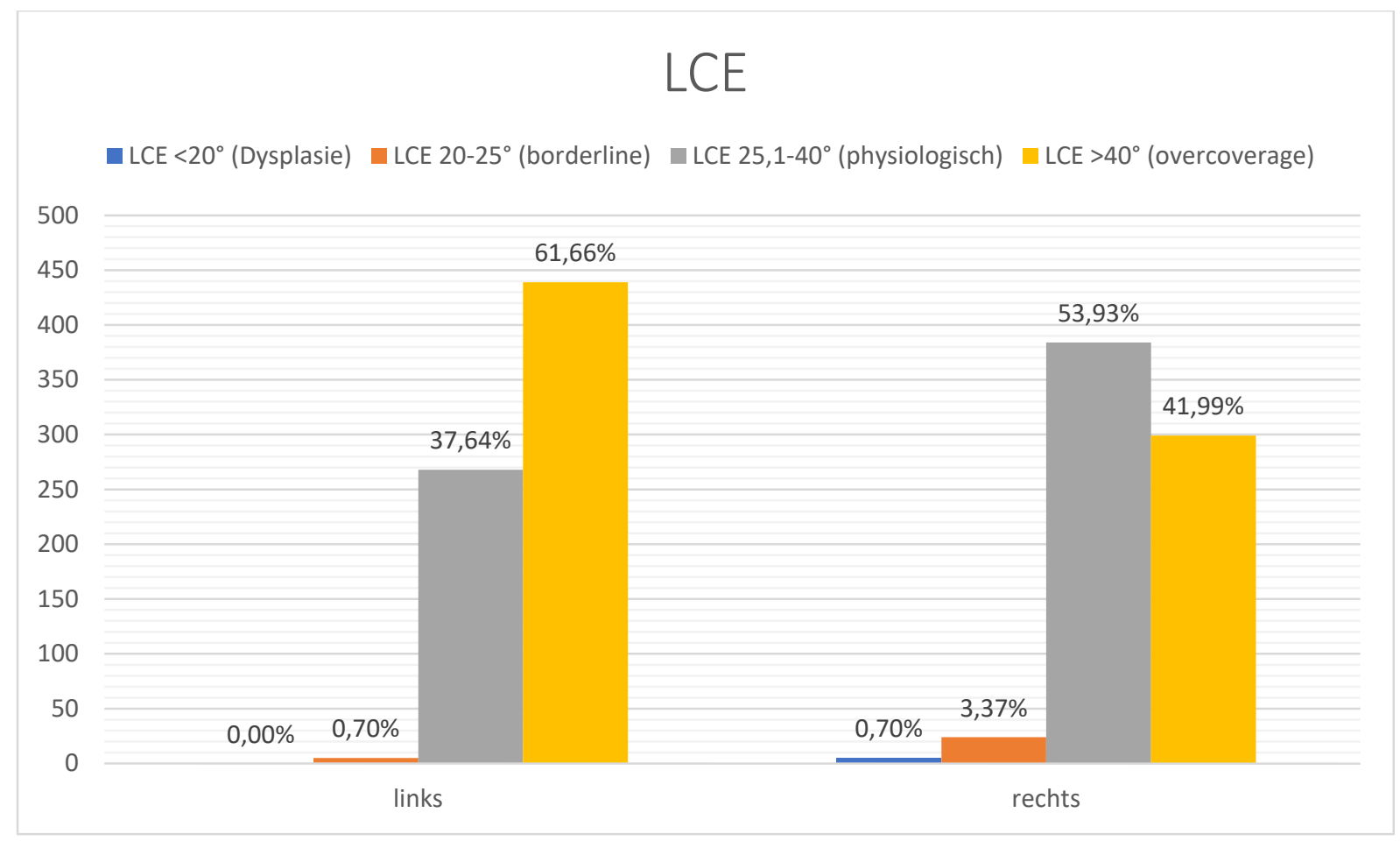

Abb. 20 Verteilung des physiologischen und pathologischen LCE-Winkels im Gesamtkollektiv nach Seite unterteilt

Bei 564 (79,21\%) rechten und bei 563 linken Hüftgelenken (79,07\%) zeigte sich eine AcetAVa $>15^{\circ}$, welcher als pathologisch angesehen wird (Abb. 21). Eine Retroversionsstellung (AcetAVa $<0^{\circ}$ ) wurde nicht gefunden. Physiologische Werte wurden in 149 (20,99\%) linken und in $148(20,79 \%)$ rechten Gelenken gefunden. 


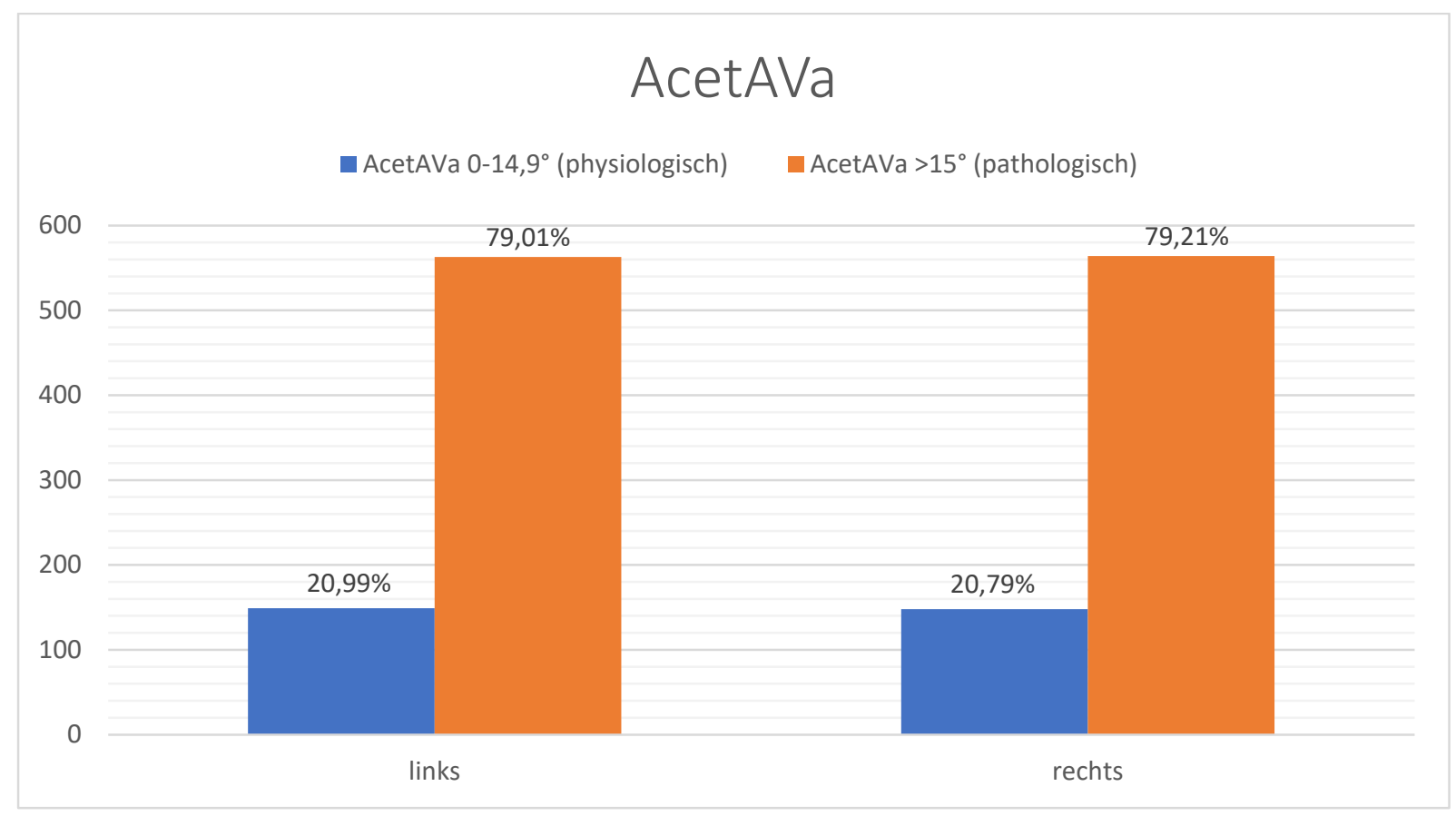

Abb. 21

Verteilung des physiologischen und pathologischen AcetAVa-Winkels im Gesamtkollektiv nach Seite unterteilt

Ein ,pathologischer“ AASA $\left(<52^{\circ}\right)$ fand sich auf der linken Hüftseite 53 Mal $(7,44 \%)$ und 31 Mal rechts (4,35\%). Ein ,pathologischer“ PASA $\left(<90^{\circ}\right)$ wurde in 77 linken $(10,81 \%)$ und 52 rechten (7,30\%) Hüftgelenken gemessen. Diese Hüftgelenke wären als dysplastisch einzustufen.

Eine pathologische Überdachung (,Overcoverage“ $\mathrm{SW}<38^{\circ}$ ) des Femurkopfes durch das Acetabulum wurde in 397 (55,76\%) linken und auch rechten Hüftgelenken gefunden. Davon konnte ein schweres Overcoverage $\left(\mathrm{SW}<34^{\circ}\right.$ ) in 198 (links; 27,81\%) respektive 159 (rechts; 22,33\%) Fällen nachgewiesen werden. Dysplasiezeichen $\left(\mathrm{SW}>43^{\circ}\right)$ zeigten 55 (7,72\%) linke und 92 (12,92\%) rechte Hüften. Physiologische Werte (SW: 38-43º) wurden in 260 (36,52\%) linken Gelenken und in 223 (31,32\%) rechten Gelenken festgestellt. Abb. 22 veranschaulicht die Messergebnisse in Form eines Diagrammes. 


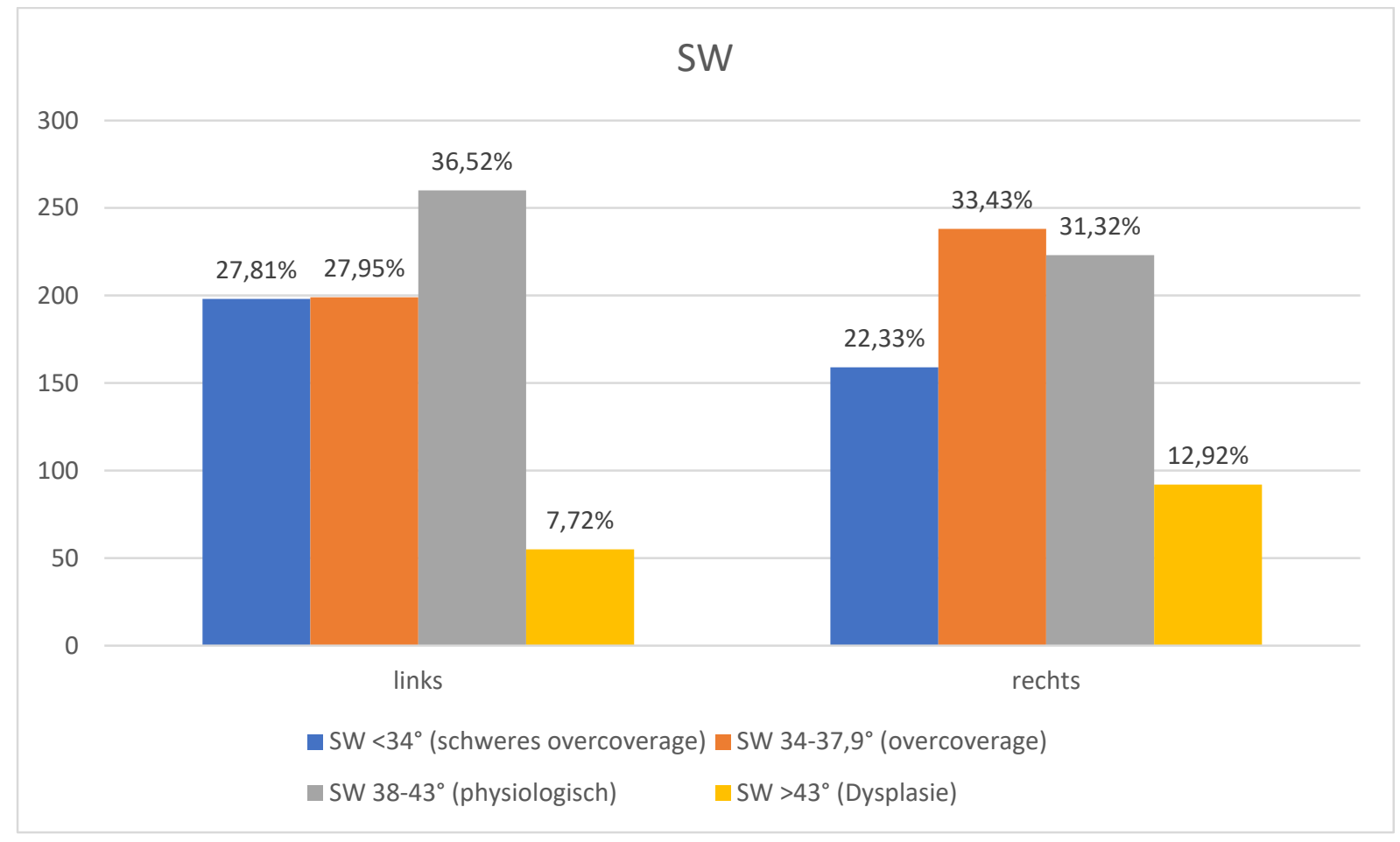

Abb. 22 Verteilung des physiologischen und pathologischen Sharp-Winkels im Gesamtkollektiv nach Seite unterteilt

Die Diskussion dieser Ergebnisse stellt die Auseinandersetzung mit der fünften Fragestellung der vorliegenden Arbeit dar. 


\section{Diskussion}

\subsection{Einfluss der Seite auf die Morphologie des Hüftgelenks}

Nur sehr wenige Studien haben bisher seitenspezifische Unterschiede für hüftmorphologische Parameter untersucht. Als aussagekräftige Vergleichsstudien können die Untersuchungen von Buller et al. (2012), Maheshwari et al. (2010) und Jiang et al. (2015) angesehen werden. Allerdings weisen alle drei Studien lediglich geringe Fallzahlen aufweisen (Buller et al. [2012] $n=115$; Maheshwari et al. [2010] $n=172$; Jiang et al. [2015] $n=466$ ) und zudem wurden nur wenige Messparameter erhoben (Buller et al. [2012]: FNV, CCD, LCE und AcetAVa; Maheshwari et al. [2010]: FNV und AcetAVa; Jiang et al. [2015]: FNV, CCD und AcetAVa). Sämtliche Daten wurden, wie in der vorliegenden Arbeit, retrospektiv aus CT-Untersuchungen generiert, sodass ein Vergleich der jeweiligen Daten sinnvoll erscheint. Jiang et al. (2015) verwendeten zu Auswertungen der CT-Scans den Centricity ${ }^{\mathrm{TM}}$ Universal Viewer - dasselbe Computerprogramm, welches auch für die Datenerhebung dieser Arbeit benutzt wurde. Die übereinstimmende Methodik macht einen Ergebnisvergleich besonders interessant und aussagekräftig.

Nachfolgend werden die seitenbezogenen Ergebnisse parameterspezifisch diskutiert.

\subsubsection{LCE}

Die Ergebnisse dieser Studie erbrachten signifikante Seitenunterschiede für die erhobenen Daten des LCEs $(p<0,0001)$. Der absolute Unterschied zwischen dem linken und rechten Mittelwert für den LCE betrug $4^{\circ}$ (links: $42,9^{\circ}$, rechts: $38,9^{\circ}$ ). Eine mögliche Erklärung für die Seitendifferenz des LCEs in der vorliegenden Studie könnte eine Verkippung des Beckens sein und somit könnte bei standardisierter Bestimmung des LCEs ein Messfehler vorliegen. Um diese Fehlerquelle ausschließen zu können, wurden Ausgleichsmessungen für mögliche Verkippungen der Becken aller Hüftgelenke vorgenommen.

Vergleichend kann nur die Studie von Buller et al. (2012) betrachtet werden, da nur in dieser der LCE seitenspezifisch untersucht wurde. Die Autoren konnten keinen signifikanten Seitenunterschied für den LCE feststellen. Buller et al. (2012) beschrieben einen Mittelwert von $37,9^{\circ}$ in den linken Hüftgelenken und von $37,4^{\circ}$ in den rechten. Somit fanden Buller et al. (2012) außerdem kleinere Messergebnisse. Interessant ist dennoch, dass trotz fehlender Signifikanz auch Buller et al. einen um $0,5^{\circ}$ größeren LCE am linken Hüftgelenk im Vergleich zum rechten fanden. Die von uns dargestellten Seitenunterschiede des LCE erscheinen daher plausibel. 
Ein seitenspezifischer Einfluss auf diesen Parameter wird durch die vorliegende Arbeit allerdings erstmalig beschrieben und ist im Hinblick auf zukünftige morphologische Studien von Bedeutung.

Ein möglicher Erklärungsansatz könnte die Seitendominanz einer Körperhälfte sein. Der Großteil der Weltbevölkerung weißt eine Rechtsseitendominanz auf (Annett 1999). Sowohl koordinative Fähigkeiten, als auch muskuläre Kraft sind auf der dominanten Seite mehr ausgebildet. Dies führt zu einem intuitiv vermehrten Gebrauch und Belastung der rechten Körperhälfte im Kindes- und Wachtumsalter (Flowers 1975).

Der LCE ist ein Parameter zur Erfassung der Überdachung des Femurkopfes durch das Acetabulum. Die Überdachung gewährleistet eine gute Gelenkführung und stellt gleichzeitig einen Luxationsschutz dar (Renkawitz et al. 2011). Eine gut ausgeprägte Muskulatur trägt ebenfalls dazu bei (Alberton et al. 2002). Unter der Annahme das eine Rechtsseitendominanz in der adoleszenten Entwicklung in einer stärker ausgeprägten Muskulatur der rechten Körperhälfte resultiert, kann vermutet werden, dass entwicklungsmorpholgisch eine geringere Überdachung des Femurkopfes nötig ist, als auf der muskelschwächeren linken Körperseite.

Die vorliegende Arbeit kann diese Hypothese bedingt durch das retrospektive Querschnittsstudiendesign nicht verifizieren. Es wäre jedoch wünschenswert dieses morphologische Phänomen der Seitendifferenz in zukünftigen prospektiven Untersuchungen weiter zu analysieren.

Die Untersuchung seitenspezifischer hüftmorphologischer Phänomene ist auch klinisch sehr relevant. Zum Beispiel wird bei einseitiger Coxarthrose häufig die kontralaterale Seite zur Referenzierung für die präoperative Endoprothesenplanung herangezogen (Renkawitz et al. 2011). Die Kenntnis über mögliche physiologische Seitenunterschiede in der Hüftmorphologie, muss daher in der präoperativen Planung berücksichtig werden. Der LCE, als Indikator für die kraniolaterale Überdachung des Femurkopfes, ist insbesondere für die Implantation einer Totalendoprothese in Bezug auf die Planung der Pfanne von Bedeutung. Das Auffräsen des Acetabulums und die Positionierung des Pfannenimplantats bestimmt die spätere Überdachung des neuen künstlichen Hüftgelenks (Wagner et al. 2012). Um ein gutes postoperatives Outcome zu erzielen, ist eine präzise Planung und Positionierung des Implantats unerlässlich (Conventry et al. 1974; Pagnano et al. 1996; Wan et al. 2008). Daher ist es wichtig, dass der Operateur sowohl für die präoperative Planung, als auch für die intraoperative Implantatpositionierung, über seitenabhängige physiologische Unterschiede des LCEs informiert ist und diese entsprechend berücksichtigen kann. 


\subsubsection{AcetAVa}

Die Messergebnisse für die AcetAVa erbrachten keine signifikanten Seitenunterschiede $(p=0,17)$. Die rechte Körperseite $\left(20,8^{\circ}\right)$ wies im Mittel einen $0,5^{\circ}$ größeren Winkel auf als die linke Seite $\left(20,3^{\circ}\right)$. Sowohl Buller et al. (2012), als auch Maheshwari et al. (2010) und Jiang et al. (2015) untersuchten die AcetAVa ebenfalls. Signifikante Seitenunterschiede fanden nur die letztgenannten Autoren.

Interessanterweise wurden in allen drei Vergleichsstudien größere Werte für die AcetAVa auf der rechten Körperseite gefunden. Buller et al. (2012) fanden linksseitig einen Winkel von 26,4 und rechtsseitig eine AcetAVA von 26,9 $9^{\circ}$. Der absolute Seitenunterschied betrug ebenso $0,5^{\circ}$, wie in der vorliegenden Arbeit. Maheshwari et al. (2010) präsentierten Winkel von 18,9 (links) und 19,2 (rechts). In der Studie von Jiang et al. (2015) fand sich auf der linken Hüftseite eine AcetAVa von $18,4^{\circ}$ und auf der rechten von $19,1^{\circ}$. Trotz der fehelenden Signifikanz der Werte in der vorliegenden Arbeit und in den Untersuchungen von Buller et al. (2012) sowie Maheshwari et al. (2010), ist ein morphologischer Seitenunterschied in Bezug auf die AcetAVa anzunehmen, da sich in allen Studien größere Werte auf der rechten Körperseite fanden. Allerdings konnten nur Jiang et al. signifikante seitenspezifische Unterschiede feststellen.

Die physiologische acetabuläre Anteversion scheint mit ca. $20^{\circ}$ richtig beschrieben zu sein, da bis auf Buller et al. auch beide anderen Autorgruppen eine ähnliche Datenverteilung messen konnten.

Ein möglicher Erklärungsansatz könnte in der unter 4.1.1 beschriebenen Rechtsseitendominanz liegen. Die AcetAVa beschreibt die Ausrichtung des Acetabulums in der sagittalen Ebene (Stem et al. 2006). Mit steigender AcetAVa nimmt die Stabilität im Hüftgelenk zu und vermindert so das Risiko einer dorsalen Luxation des Femurkopfes aus dem Acetabulum (Lewinnek et al. 1978; Widmer und Zurfluh 2004). Es wäre denkbar, dass bei vermindertem LCE, der eine höhere Beweglichkeit des Hüftgelenks ermöglicht, kompensatorisch zur dorsalen Stabilisierung eine größere acetabuläre Anteversion im Hüftgelenk notwendig ist. Darüber hinaus ist auch denkbar, dass ein Hüftgelenk, welches stärkeren Belastungen ausgesetzt ist, eine bessere ossäre Stabilisierung benötigt und sich daher eine vermehrte acetabuläre Anteversion im dominanten Hüftgelenk entwickelt. Die Fortführung solcher Theorien sollte in biomechanischen Simulationsstudien überprüft und validiert werden.

\subsubsection{AASA und PASA}

Interessanterweise konnten in der vorliegenden Arbeit nur für den PASA $(p=0,007)$, nicht aber für den AASA ( $\mathrm{p}=0,31)$, signifikante Seitenunterschiede festgestellt werden. 
Der AASA betrug auf der linken Hüftseite $65,6^{\circ}$ und auf der rechten $65,2^{\circ}$ (absoluter Werteunterschiede $0,4^{\circ}$ ). Die Untersuchungen des PASAs zeigten im Mittel Werte von 106, $0^{\circ}$ (linkseitig) bzw. $107,6^{\circ}$ (rechtsseitig). Der absolute Werteunterschied zwischen den seitenspezifischen Mittelwerten lag bei $1,6^{\circ}$.

Keine der Referenzstudien beschäftigte sich mit einem seitenspezifischen Vergleich der morphologischen Unterschiede in Bezug auf den AASA bzw. PASA. Die vorliegende Arbeit präsentiert daher erstmalig Messergebnisse zu dieser Fragestellung. Daher sind schon allein diese rein deskriptiven Messwerte für die hüftmorphologische Grundlagenforschung von Bedeutung und können zukünftig als Referenz herangezogen werden.

Anhand des AASAs und des PASAs kann die axiale Überdachung des Hüftkopfes festgestellt werden (Harrasser et al. 2016). Dabei beschreibt der anteriore acetabuläre Sektorwinkel (AASA) die ventrale Überdachung und der posteriore acetabuläre Sektorwinkel (PASA) die dorsale Überdachung des Femurkopfes.

Interessanterweise zeigten die Ergebnisse dieser Studie einen signifikant größeren PASA auf der rechten, als auf der linken Hüftseite, während der AASA links größer als rechts war. Diese Messergebnisse unterstützen die bereits diskutierten Ergebnisse in 4.1.2 und deren Interpretation. Die tendenziell in 4.1.2 vorgestellte größere AcetAVa basiert vor allem auf einer zunehmenden dorsalen Überdachung des Femurkopfes und weniger auf einer Abnahme des vorderen Pfannenrandes. Diese Erkenntnis unterstreicht die Überlegung, dass mit einer vergrößerten AcetAVa vor allem eine dorsalseitig verbesserte Stabilität des Hüftgelenks erreicht werden würde und daher auch der posteriore und nicht der anteriore Sektorwinkel zunimmt.

Erstmalig kann eine solche Diskussion anhand der in der vorliegenden Studie erhobenen Messwerte thematisiert werden. Diese Erkenntnisse sind daher für morphologische Grundlagenforschung von besonderem Wert.

Aber auch im klinischen Alltag ergibt sich zum Beispiel bei der Implantation einer Hüft-TEP aus dem oben Beschriebenen eine Relevanz. Die bestehenden Zusammenhänge zwischen Überdachungsparametern und Pfannenpositionierung sind bei der Wahl des Inlaydesigns zu berücksichtigen (Widmer 2007; Mihalko et al. 2009; Beverland et al. 2016). Die Entscheidung ob ein Inlay beispielsweise planar, mit $10^{\circ}$ Randüberhöhung oder mit $20^{\circ}$ Randüberhöhung eingebaut wird, wird unter anderem an der AcetAVa des Pfannenimplantats bemessen. Diese wird allerdings häufig nur per Augenmaß geschätzt (Wagner et al. 2012). 
Ein genauer radiologischer Abgleich unter Berücksichtigung des AASAs und PASAs könnte die postoperative Luxationsrate durch eine bessere Pfannenpositionierung oder aber korrigierendes Inlaydesign verbessern.

\subsubsection{AI}

Für den AI wurde linksseitig im Mittel ein Winkel von $0,1^{\circ}$ und rechtsseitig von $0,0^{\circ}$ gefunden (absoluter Wertunterschied $\left.0,1^{\circ}\right)$. Die Ergebnisse unterschieden sich nicht signifikant $(p=0,87)$. In keiner der angeführten Vergleichsstudien wurde der AI untersucht.

Durch den relativ kleinen Messunterschied und die fehlende Signifikanz muss davon ausgegangen werden, dass keine Seitenspezifität in Bezug auf den AI im Hüftgelenk vorliegt.

Da der AI erstmalig in einem großen Patientenkollektiv untersucht wurde und sich aktuell keine Vergleichsliteratur findet, sollten die absoluten Zahlenwerte zukünftig zumindest als Referenzwerte für weitere Studien genutzt werden.

\subsubsection{SW}

In der vorliegenden Arbeit zeigte sich der mittlere SW in den linken Hüftgelenken bei $36,6^{\circ}$ und in den rechten Hüftgelenken bei 37,6 $6^{\circ}$ (absoluter Werteunterschied 1,0 $0^{\circ}$. Es konnte ein signifikanter Seitenunterschied festgestellt werden $(p=0,0003)$.

Auch der SW wurde in den angeführten Vergleichsstudien nicht untersucht, sodass abermals erstmalig eine Seitenabhängigkeit des SW in der vorliegenden Arbeit beschrieben wird.

Anhand des SW kann die Ausrichtung des Acetabulums in der koronaren Ebene erfasst werden (Tannast et al. 2015). Somit wird auch durch diesen Winkel ein Aspekt der Überdachung des Femurkopfes durch die Pfanne erfasst, wobei größere Werte eine geringere kraniale Überdachung bedeuten. Wie auch bereits beim LCE in 4.1.1 beschrieben, ist eine geringere kraniale acetabuläre Überdachung auf der rechten Seite zu finden. Nach dem Schema der in dieser Arbeit beschriebenen Theorie der Seitendominanz wäre es möglich, dass bei stärker ausgeprägter Muskulatur auf der dominanten Körperseite das rein anatomische Luxationsrisiko im Zuge der Entwicklung ansteigt, um eine höhere Mobilität in Bezug auf das Bewegungsausmaß des Hüftgelenks zu erreichen. Das Luxationsrisiko würde bei ansteigendem SW rein hypothetisch in die kraniolaterale Richtung ansteigen, aber durch die bessere Muskelführung reell betrachtet nicht erhöht sein. Diese Ausführungen sind sowohl für die morphologische Grundlagenforschung, als auch für den klinischen Alltag von Bedeutung. 
Im Bereich der Hüftendoprothesenimplantation ist die Inklination der Pfanne ein essentieller Faktor für ein positives Operationsergebnis mit guter Bewegungsfunktion und geringem Luxationsrisiko (Widmer 2016). Da Patienten zum Teil auch beidseitig einen Gelenkersatz benötigen, wäre es sinnvoll, den in dieser Studie nachgewiesenen Seitenunterschied des SW zukünftig für die Prothesenplanung und die Implantation zu berücksichtigen.

\subsubsection{FNV}

Bei der FNV fand sich eine absolute Seitendifferenz von $0,8^{\circ}$. Es zeigte sich dabei kein signifikanter Seitenunterschied $(p=0,11)$. Dabei konnte im linken Hüftgelenk ein größerer Mittelwert $\left(12,1^{\circ}\right)$ für die FNV im Vergleich zum rechten Hüftgelenk $\left(11,3^{\circ}\right)$ gemessen werden.

Alle drei angeführten Vergleichsstudien untersuchten ebenfalls eine Seitenabhängigkeit der FNV. Buller et al. (2012) beschrieben einen Mittelwert der FNV von 4,7 in linken Hüften und von $4,5^{\circ}$ in den rechten. Die Werte waren nicht signifikant unterschiedlich $(p=0,45)$. Auch Jiang et al. konnten keinen signifikanten Seitenunterschied nachweisen (links: 10,93; ${ }^{\circ}$ rechts: $10,41^{\circ}$; $\mathrm{p}=0,175$ ). Maheshwari et al. (2010) fanden linksseitig einen Mittelwert von 8,7 $7^{\circ}$ und rechtsseitig von $7,4^{\circ}$. Auffällig ist, dass auch in den drei anderen Studien, nicht signifikant, aber trotzdem tendenziell größere Mittelwerte für die FNV in den linken Hüftgelenken gefunden worden.

Werden die absoluten Werte der Ergebnisse aller Studien verglichen fällt eine Diskrepanz auf. Die Messwerte von Buller et al. (2012) erscheinen mit knapp $5^{\circ}$ dabei sehr klein. Die Ergebnisse der vorliegenden Arbeit und die der anderen Studien liegen näher aneinander. In keiner Publikation der anderen Studien ist das genaue Messverfahren beschrieben. Möglicherweise könnten Buller et al. (2012) die FNV falsch bestimmt haben. Wie unter 2.3 beschrieben, errechnet sich die FNV aus einem superioren und einem inferioren Wert. Entscheidend ist die Kondylenrotation. Bei einer Außenrotationstellung muss die inferiore FNV von der superioren subtrahiert werden. Liegt eine Innenrotation vor, werden beide Werte addiert. Möglicherweise wurde fälschlicherweise auch bei Innenrotationsstellungen von Buller et al. (2012) die inferiore von der superioren FNV subtrahiert. Dies würde zu kleineren Mittelwerten führen.

Die FNV beschreibt den Antetorsionswinkel (Winkel zwischen Schenkelhalsachse und Femurkondylenebene. Dabei bedingt eine größere FNV ein vermehrt innenrotiertes Gangbild und eine kleinere FNV ein vermehrt außenrotiertes Gangbild (Hetsroni et al. 2013). Eine Innenrotationsstellung ist dabei mehr stabilitätsgefährdend für das Kniegelenk als eine Außenrotationsstellung (Hasler und Hefti 2015). 
Aufbauend auf der in dieser Arbeit thematisierten Theorie der Seitendominanz und der vermuteten stärkeren muskulären Ausprägung auf der dominanten Körperseite, wäre es vorstellbar, dass sich durch dieses physiologische Ungleichgewicht, die muskelschwächere Seite, bedingt durch das Übergewichts an Muskulatur, in eine anatomisch instabilere morphologische Form entwickelt. Dabei ist nicht zwangsläufig von einer konkreten pathologischen Instabilität auszugehen. Es wird rein das relative Ausmaß der Stabilität betrachtet. Konkret würde das bedeuten, dass die erhöhte FNV auf der muskelschwächeren linken Seite eine entwicklungsmorphologische Folge eines physiologischen Seitenungleichgewichts an Muskulatur wäre.

Um diese Überlegungen $\mathrm{zu}$ belegen bedarf es jedoch weiterer Studien, die diese Zusammenhänge gezielter untersuchen.

\subsubsection{CCD}

Die vorliegende Arbeit zeigte für den CCD einen signifikanten $(p<0,0001)$ Seitenunterschied von 1,6 (links: $132^{\circ}$; rechts $128,4^{\circ}$ ). In den Studien von Buller et al. (2012) und Jiang et al. (2015) wurde der CCD ebenfalls seitenabhängig untersucht. Die erstgenannte Autorengruppe präsentierte linksseitig einen Mittelwert von $127,8^{\circ}$ und rechtsseitig von $128,7^{\circ}$, allerdings ohne signifikanten p-Wert $(0,86)$. Jiang et al. (2015) stellten einen signifikanten Seitenunterschied fest $(p<0,001)$. Sie fanden in den untersuchten linken Hüften einen CCD von $133,97^{\circ}$ und in den rechten von $132,4^{\circ}$. Ebenso wie in der vorliegenden Arbeit wies dabei die linke Körperseite den größeren Winkel gegenüber der rechten auf. Da beide Studien signifikante Werte nachweisen konnten und auch Buller et al. (2012) unterschiedliche seitenbezogene Ergebnisse zeigen konnten, ist eine Seitenabhängigkeit des CCDs wahrscheinlich. Die signifikanten Seitenunterschiede aus der vorliegenden Studie sowie die aus der Studie von Jiang et al. (2015) weisen beide linksseitig größere CCD-Winkel auf. Da Buller et al. (2012) keine signifikanten Unterschiede bei größerem rechtsseitigen CCD feststellen konnten, sollten eher die linksseitig größeren Ergebnisse als korrekt angesehen werden. Außerdem ist zu berücksichtigen, dass das Studienkollektiv von Buller et al. (2012) mit 115 eingeschlossenen Patienten vergleichsweise klein ist. Jiang et al. (2015) untersuchten 466 Probanden und in der vorliegenden Arbeit wurden für die finale Auswertung, Untersuchungen von 712 Patienten inkludiert. Die kleine Stichprobe könnte auch ein Grund für die divergenten Ergebnisse von Buller et al. (2012) sein.

Eine mögliche Erklärung für den Einfluss der Körperseite auf die Größe des CCDs könnte sich abermals in der Theorie der Seitendominanz finden. 
Wird davon ausgegangen, dass die koordinativ sowie motorisch stärkere rechte Seite auch im Alltag vermehrt eingesetzt und damit verstärkt belastet würde, wären die axial auf den Schenkelhals wirkenden Kräfte in der Summe größer als auf der linken Seite. Dadurch würde es morphologisch zu einem „Abkippen“ des Schenkelhalses in Richtung des Femurschaftes kommen, wodurch der CCD kleiner werden würde.

Analog zur FNV ist auch der CCD essentiell für die präoperative Prothesenplanung für einen Ersatz des Hüftgelenks (Ellison 2012; Yoshimine 2005; Hisatome und Doi 2011; Müller et al. 2011). Solange kein pathologischer CCD präoperativ vorbestände, könnte der individuell physiologische vorliegende Winkel als Orientierung für den später implantierten Prothesenschaft dienen. Aktuell werden Standardimplantate verwendet, die einen individuellen CCD nicht berücksichtigen (Wagner et al. 2012). Ein individuell-morphologisches Implantat könnte, wie unter 4.1.6 beschrieben, zu einem besseren postoperativen Outcome, mit schnellerer Mobilisation sowie Rehabilitation und einer größeren Bewegungsmobilität führen.

Des Weiteren orientieren sich präoperative Prothesenplanungen mit dem Ziel einer kurativen Therapie von Schenkelhalsfrakturen an der gesunden kontralateralen Seite (Wagner et al. 2012). Vor allem in diesem Zusammenhang sollten die seitenabhängigen unterschiedlichen CCD-Werte berücksichtig werden.

Diese Überlegungen sollten zukünftig bei der Entwicklung neuer Implantatdesigns berücksichtigt werden.

\subsubsection{AaA und $\mathrm{S} \alpha \mathrm{A}$}

Keine der angeführten Referenzstudien stellt einen Seitenvergleich des A $\alpha$ A oder des S $\alpha \mathrm{A}$ an. Die vorliegende Arbeit präsentiert erstmalig eine solche Untersuchung.

Für den AaA zeigte sich ein signifikanter $(\mathrm{p}<0,0001)$ Seitenunterschied von $3,9^{\circ}$. In linken Hüftgelenken wurde ein Mittelwert von 39, $3^{\circ}$ festgestellt und in den rechten von $43,2^{\circ}$. Auch der SaA unterschied sich seitenabhängig signifikant $(\mathrm{p}<0,0001)$ und wies ebenfalls in der rechten Hüfte den größeren Winkel auf (links: 44,9 ; rechts: 47,3; absoluter Wertunterschied: $\left.2,4^{\circ}\right)$

Diese Ergebnisse sollten auf rein deskriptiver morphologischer Ebene zukünftig berücksichtig werden. Vor allem der $\mathrm{S} \alpha \mathrm{A}$ ist ein sehr spezieller Parameter, der in der aktuellen Literatur bislang nur wenig beschrieben wurde. Dem A $\alpha$ A wird eine prädiktive Funktion zur Detektion eines Cam-Impingements zugeschrieben (Nötzli et al. 2002). Die Aussage, dass dieser, physiologischerweise, eine Seitenabhängigkeit aufzuweisen scheint, ist hingegen neu. 
Möglicherweise spielt auch für den $\mathrm{A} \alpha \mathrm{A}$ und den $\mathrm{S} \alpha \mathrm{A}$ die axiale Belastung des Hüftgelenks eine Rolle. Denkbar wäre ein Ansteigen beider Winkel durch die axial wirkenden Kräfte, welche in der Summe auf der dominanten Körperseite größer wären. Interessant ist diese Beobachtung aus klinischer Sicht auch hinsichtlich eines Hüftgelenkimpingement. Werden die Ergebnisse des LCEs und SWs für die kraniolaterale Überdachung der Pfanne berücksichtigt, fällt auf, dass im linken Hüftgelenk zwar eine größere kraniolaterale Überdachung des Acetabulums vorliegt, gleichzeitig aber der $\mathrm{S} \alpha \mathrm{A}$ des Schenkelhalses kleiner ist und so ein Impingment zwischen Pfanne und Schenkelhals nicht auftreten kann. Für die rechte Seite verhält sich die Beschreibung genau gegenteilig. Rechts besteht eine geringere Pfannenüberdachung bei allerdings ausgeprägterer superiorer Schenkelhalskonfiguration. Beim vorderen Impingement kommt es $\mathrm{zu}$ einem Anstoßen zwischen dem ventralen Pfannenrand und dem anterioren Schenkelhals (Austin et al. 2008; Hartofilakidis et al. 2011; Lerch et al. 2018). Die Ergebnisse der vorliegenden Studie beschreiben durch den AASA einen kleineren ventralen Pfannenrand, wobei sich der anteriore Schenkelhals verbreitert zeigt (A $\alpha A)$. Gegensätzlich verhält sich das linke Hüftgelenk. Insgesamt scheinen dem zur Folge morphologische Kompensationsmechanismen vorzuliegen, sodass die Wahrscheinlichkeit für ein Impingement zwischen Pfanne und Schenkelhals verringert wird.

Um diese Vermutungen zu bekräftigen, bedarf es jedoch weiterer Studien, die sich gezielter mit einer solchen Fragestellung auseinandersetzten.

\subsubsection{AO, FO und GO}

Sowohl AO $(\mathrm{p}=0,009)$, als auch FO $(\mathrm{p}<0,0001)$ und GO $(\mathrm{p}<0,0001)$ wiesen signifikante Seitenunterschiede auf. Alle drei Parameter zeigten rechtsseitig größere Mittelwerte als linksseitig. Für das AO betrug diese in den linken Hüften 31,5 mm und in den rechten 32,2 mm (absoluter Wertunterschied: 0,7 mm). Beim FO fanden sich analog $39 \mathrm{~mm}$ bzw. $41 \mathrm{~mm}$ (absoluter Wertunterschied $2 \mathrm{~mm}$ ) und beim GO 70,5 mm bzw. 73,2 $\mathrm{mm}$ (absoluter Wertunterschied $(2,7 \mathrm{~mm})$.

In keiner der angeführten Vergleichsstudien finden sich Untersuchungen bezüglich der Offset-Parameter, sodass zum ersten Mal eine Seitenabhängigkeit von diesen beschrieben wird. Ein Erklärungsansatz findet sich erneut in der in dieser Arbeit thematisierten Theorie der Seitendominanz. Je größer AO und FO (somit auch GO) werden, desto gleichmäßiger verteilen sich die auf das Hüftgelenk wirkenden Kräfte. 
Unter der Annahme, dass die koordinativ und motorisch besser ausgebildete Körperhälfte im Alltag häufiger eingesetzt werden würde (egal ob bewusst oder unbewusst) und somit auch einer höheren Belastung ausgesetzt wäre, könnte ein morphologischer Kompensationsmechanismus in einem Anwachsen der Offset-Parameter liegen. In diesem Fall wären die anatomischen Strukturen besser an die höhere Belastung angepasst.

\subsubsection{0 ØA, ØF und F/A}

Für ØA $(p=0,48)$ und $\varnothing F \quad(p=0,16)$ konnten keine signifikanten Seitenunterschiede nachgewiesen werden, für das $\mathrm{F} / \mathrm{A}(\mathrm{p}=0,001)$ hingegen schon. Es zeigten sich für den ØA linksseitig Mittelwerte von 58,4 mm und rechtsseitig von 58,5 mm (absoluter Wertunterschied 0,1 mm). Beim ØF waren es 47,8 $\mathrm{mm}$ in den linken Hüften und 47,4 $\mathrm{mm}$ in den rechten (absoluter Wertunterschied 0,4 mm). Das F/A betrug 82,0\% (links) bzw. 81,2\% (rechts), sodass ein absoluter Unterschied von $0,8 \%$ festgestellt werden konnte.

Erstmalig wird in einem großen Patientenkollektiv eine Seitenabhängig des ØA, ØF und des F/A gezielt untersucht. In den angeführten Vergleichsstudien wurden keine seitenspezifischen Unterschiede für diese Parameter ermittelt.

Aufgrund der fehlenden Signifikanz der Einzeldurchmesser von Acetabulum und Femurkopf ist eine morphologische Seitenabhängigkeit nicht nachzuweisen. Dass der Quotient aus beiden Werten signifikante Unterschiede aufweist, könnte an der Kollektivgröße liegen. Eine naheliegende Begründung für die Ergebnisse ist nicht zu finden, da ØA zwar rechtsseitig größer ist, ØF allerdings kleiner. Durch die vorliegende Arbeit kann eine Kausalität für diese morphologischen Beschaffenheiten nicht hergestellt werden. Die rein deskriptiven Werte sollten dennoch als Referenzwerte für weitere Studien berücksichtigt werden.

\subsubsection{Arthrosegrad}

In der vorliegenden Arbeit fanden sich keine signifikanten $(p=0,46)$ Seitenunterschiede hinsichtlich des Arthrosegrades. Die Vergleichsstudien präsentieren keine Referenzwerte. Stadienabhängig zeigt sich ein relativ homogenes Bild. Linksseitig fanden sich 12,2\% der untersuchten Gelenke mit einem KL 0, 32,4\% mit einem KL 1, 49,4\% mit einem KL 2, 5,2\% mit einem KL 3 und 0,7\% mit einem KL 4. Für die rechten Hüften präsentierte sich folgende Verteilung: 14,3\% KL 0, 33,9\% KL 1, 45,1\% KL 2, 5,6\% KL 3 und 1,1\% KL 4.

Aufgrund der fehlenden Signifikanz der Messwerte und der ausgeglichenen Verteilung in den einzelnen Arthrosestadien ist eine Seitenabhängigkeit nicht nachzuweisen. 
Es ist anzumerken, dass der Arthosegrad in dieser Studie primär aus Gründen der Validierung eines möglichst asymptomatischen Patientenkollektivs erhoben wurde und nicht gezielt auf Seitenabhängigkeit gescreent wurde. Für eine solche Analyse wäre ein Kollektiv mit deutlich höherer Arthroserate und einer ausgeglichenen Größenverteilung stadienübergreifend notwendig. Eine solche Analyse war nie Ziel dieser Arbeit. Daher dürfen die ermittelten Werte nicht falsch interpretiert werden und sollten rein deskriptiv betrachtet werden.

\subsubsection{Schlussfolgerungen}

Werden sämtliche zuvor diskutierten Aspekte gesamtheitlich betrachtet lässt sich Folgendes festhalten.

Für den LCE, den PASA, den SW, den CCD, den A $\alpha$ A, den S $\alpha$ A, das AO, das FO und das GO konnten signifikante Seitenunterschiede festgestellt werden. Eine mögliche Ursache könnte in der motorischen sowie koordinativen Dominanz liegen.

Für die AcetAVa, den AASA und die FNV fanden sich zwar keine signifikanten Seitenunterschiede, aber die ermittelten Werte fügen sich sehr gut in den beschriebenen Erklärungsansatz ein. Außerdem sind Ergebnisse mit denen aus Referenzstudien vergleichbar, die signifikante Messwerte ermitteln konnten.

Das F/A erbrachte zwar auch signifikante Seitenunterschiede, ließ sich allerdings nicht plausibel in den Erklärungsansatz einfügen.

Auch die nicht signifikanten Ergebnisse von AI, ØA und ØF sollten trotz dessen zukünftig als rein deskriptive Referenz für weitere Studien genutzt werden. Die thematisierte Theorie der Seitendominanz ist grundlagenmorphologisch von enormer Bedeutung und muss durch weitere Untersuchungen genauer analysiert werden. Eine ausführlichere Auseinandersetzung mit der ätiologischen Morphogenese erscheint außerordentlich untersuchungsbedürftig. Den Grundstein dafür hat die vorliegende Studie gelegt.

Besonders interessant sind die diskutierten Erkenntnisse und Überlegungen auch für den Bereich der Endoprothetik. Präoperative Planung und postoperativer Heilungsverlauf könnten durch seitenbezogene Orientierung an individuell-morphologischen Parametern verbessert werden und damit einen großen klinischen Benefit erzielen.

Abschließend muss festgehalten werden, dass die vorliegende Studie erstmalig mit hoher Fallzahl, einen Seitenvergleich für eine große Anzahl von hüftmorphologischen Parametern mittels dreidimensionaler Bildgebung angestellt hat. 


\subsection{Einfluss des Alters auf die Morphologie des Hüftgelenks}

Wie in der Einleitung erläutert, ist die morphologische Entwicklung des Hüftgelenks von Geburt bis zum Ende der Adoleszenz sehr gut untersucht. Aktuell gibt es nur sehr wenige Studien, die den Einfluss des Alters auf die Morphologie des Hüftgelenks analysiert haben. Zu den aussagekräftigen Referenzstudien zählen die Untersuchungen von Jiang et al. (2015) und von Miyasaka et al. (2017). Die Studie von Miyasaka et al. wies mit nur 120 eingeschlossenen Patienten ein eher kleines Kollektiv auf. Allerdings wurde eine hohe Anzahl an morphologischen Parametern untersucht, sodass ein Vergleich mit der vorliegenden Studie sehr interessant erscheint. Die Autoren nutzten für die Datenerhebung ebenfalls CT-Bilder.

Beide Vergleichsstudien unterteilten ihre Kollektive lediglich in zwei Altersgruppen. Jiang et al. (2015) grenzten zwischen Patienten mit $<60$ bzw. $>60$ Lebensjahren und Miyasaka et al. (2017) zwischen $<50$ bzw. $>50$ Jahren ab. Die Einteilung der vorliegenden Arbeit in vier Altersgruppen (19-40, 40-60, 60-80, >80) ist wesentlich genauer und ermöglicht, morphologische Veränderungen des Hüftgelenks bestimmten Altersbereichen klarer zuzuordnen. Gleichzeitig wurde in der vorliegenden Studie eine deutlich höhere Anzahl an Patienten (n=712) bzw. Hüftgelenken (n=1424) untersucht.

Zusammenfassend präsentiert die vorliegende Arbeit demzufolge erstmalig eine detaillierte Betrachtung altersabhängiger Veränderungen in der Morphologie des Hüftgelenks in einem großen Studienkollektiv.

Nachfolgend werden die Ergebnisse der vorliegenden Studie parameterspezifisch diskutiert.

\subsubsection{LCE}

Es konnten signifikante Unterschiede zwischen den vier Altersgruppen für den LCE festgestellt werden $(\mathrm{p}<0,0001)$. Dabei zeigte sich ein Anstieg der Winkelgröße von der jeweils kleineren

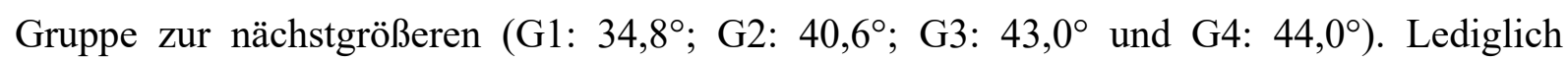
Miyasaka et al. (2017) untersuchten ebenfalls eine Altersabhängigkeit des LCE. Die Daten wurden außerdem geschlechtsabhängig dargestellt. In der vorliegenden Arbeit wurden die Ergebnisse geschlechtsunabhängig untersucht, um die alleinige Auswirkung des Alters auf die Morphologie des Hüftgelenks zu untersuchen. Mögliche Unterschiede zwischen den Geschlechtern wurden ebenfalls untersucht und werden im Kapitel 4.3 diskutiert. Die Darstellung von Miyasaka et al. (2017) beinhaltet demnach zwei Faktoren, die möglicherweise einen morphologischen Einfluss besitzen. Dies erschwert einen Vergleich. Eventuell könnte es zu einer Verzerrung der Untersuchungsdaten gekommen sein. 
Miyasaka et al. (2017) fanden sowohl bei Männern als auch bei Frauen in der Altersgruppe $<50$ Jahre einen kleineren LCE als in der Gruppe $>50$ Jahre (Männer: 28,5 bzw. 36 ${ }^{\circ}$; Frauen: 27,7 bzw. $35,7^{\circ}$ ). In beiden Gruppen waren die Messunterschiede signifikant (jeweils $p<0,001$ ). Demzufolge konnte auch diese Autorengruppe eine Zunahme des LCEs mit ansteigendem Alter feststellen. Es muss dennoch festgehalten werden, dass die absoluten Messwerte von Miyasaka et al. (2017) deutlich kleiner sind, als die der vorliegenden Arbeit. Die Differenz zwischen den beiden jüngsten Gruppen ( $<40$ Jahre in der vorliegenden Arbeit und $<50$ Jahren in Miyasaka et al. [2017]) beträgt $6,3^{\circ}$. Die geringe Kollektivgröße der Referenzstudie ( $\left.\mathrm{n}=120\right)$ könnte die Begründung für diese Diskrepanz sein.

Die Zunahme des LCEs mit dem Alter lässt sich durch degenerative Prozesse im Bereich der Pfanne erklären, die einerseits zu einer Verknöcherung des Labrum acetabulare führen und andererseits auch zu osteophytären Anbauten, die insbesondere kraniolateral auftreten können (Li et al. 1988; Croft et al. 1990). Da allerdings in dem untersuchten Kollektiv wenige Hüftgelenke mit Arthrose vorliegen, kann der degenerative Einfluss nicht allein ursächlich sein. Als weitere mögliche Ursache für die gezeigte Altersabhängigkeit des LCE käme die relative Abnahme an Muskelmasse im Zuge des Alterungsprozesses in Frage (Cruz-Jentoft et al. 2010). Dieser Prozess wird als Sarkopenie beschrieben (Rosenberg 1997; Roubenoff und Hughes 2000; Ali und Garcia 2014). Ausgehend von der Überlegung, dass eine gut ausgebildete Muskulatur als Luxationsschutz fungiert, wäre es denkbar, dass der Femurkopf in der morphologischen Entwicklung vermehrt überdacht wird, um dem ansteigenden Luxationsrisiko entgegenzuwirken bzw. dieses auszugleichen. Letztlich ist auch eine physiologische Veränderung der Pfanne durch ossäre Anbauten und damit vergrößerter Kontaktfläche als Reaktion auf eine altersabhängige veränderte Krafteinleitung in das Hüftgelenk denkbar.

Um diese Thesen zu untermauern oder zu widerlegen, sollten in der Zukunft prospektive großangelegte Studien durchgeführt werden.

Unabhängig von der Ursache zeigt die vorliegende Arbeit erstmalig, dass sich die morphologische Entwicklung der Hüftgelenkspfanne auch nach Abschluss der Adoleszenz fortsetzt.

\subsubsection{AcetAVa}

Auch für die AcetAVa konnten altersabhängige signifikante Messunterschiede zwischen den vier Gruppen nachgewiesen werden $(\mathrm{p}<0,0001)$. Dabei zeigte sich ebenso wie beim LCE eine Zunahme der Winkelgröße von der jeweils jüngeren Patientengruppe zur nächst älteren (G1: $18,5^{\circ} ; \mathrm{G} 2: 20,4^{\circ} ; \mathrm{G} 3: 20,9^{\circ}$ und $\mathrm{G} 4: 23,3^{\circ}$ ). 
Sowohl Miyasaka et al. (2017), als auch Jiang et al. (2015) untersuchten die Auswirkungen des Alters auf die Morphologie der AcetAVa. Die erstgenannte Autorengruppe fand bei den Männern im Alter unter 50 Jahren eine AcetAVa von 15,9 $9^{\circ}$ und bei denen über 50 Jahren von 19, $0^{\circ}$. Die Auswertung der Frauengruppe erbrachte Werte von 19,5 $5^{\circ}$ (<50 Jahre) bzw. 19,9 (>50 Jahre). In der Gruppe der männlichen Patienten konnte ein signifikanter Messunterschied festgestellt werden $(p=0,027)$, in der der weiblichen hingegen nicht $(p=0,731)$.

Jiang et al. (2015) präsentierten bei den Patienten unter 60 Jahren eine AcetAVa von 17,79. Bei den älteren Untersuchten ( $>60)$ ergab sich ein Mittelwert von 19,39 ${ }^{\circ}$. Es zeigte sich ein signifikanter Messunterschied $(\mathrm{p}<0,001)$.

Somit konnten alle drei Studien zeigen, dass bei zunehmendem Alter die Winkelgröße der AcetAVa zunimmt. Anders als beim LCE liegen die Messwerte der vorliegenden Studie deutlich näher an denen der Vergleichsstudien, was die Messgenauigkeit aller Studien bekräftigt. Die morphologische Entwicklung der AcetAVa setzt sich demzufolge ebenfalls nach Abschluss der Adoleszenz fort.

\subsubsection{AASA und PASA}

Sowohl für den AASA als auch für den PASA konnten signifikante altersabhängige Messunterschiede festgestellt werden (jeweils $\mathrm{p}<0,0001$ ). Der posteriore Winkel stieg von jeder kleineren Altersgruppe zur nächst größeren in der Gradzahl an (G1: 100,0; G2: 106,0; G3: 108,7; G4: $112,6^{\circ}$ ). Beim anterioren Winkel waren zwar G2 und G3 jeweils größer als die jüngere Patientengruppe, G3 wies jedoch einen größeren AASA auf als G4 (G1: 63,4 , G2: $\left.65,6^{\circ}, \mathrm{G} 3: 67,1^{\circ}, \mathrm{G} 4: 65,7^{\circ}\right)$.

Auch Miyasaka et al. (2017) untersuchten die altersabhängige Entwicklung von AASA und PASA. Bei den männlichen Patienten unter 50 Jahren fanden sie einen AASA von $58,8^{\circ}$ und einen PASA von $90,5^{\circ}$. Bei den Männern über 50 zeigten sich Winkel von $63,2^{\circ}$ bzw. $98,1^{\circ}$. Sowohl für den AASA $(p=0,001)$ als auch für den PASA $(p<0,001)$ konnten die Autoren signifikante Messunterschiede feststellen. In der jüngeren Patientengruppe der Frauen präsentierten Miyasaka et al. (2017) Werte von 55,2 $2^{\circ}$ (AASA) und 94,6 $6^{\circ}$ (PASA). In der älteren Gruppe konnte ein AASA von 59,1 ${ }^{\circ}$ sowie ein PASA von 99, $1^{\circ}$ gemessen werden. Auch hier konnten signifikante Unterschiede festgestellt werden (AASA: $p=0,009$ und PASA: $p=0,002$ ).

Die Vergleichsstudie bekräftig die Ergebnisse der vorliegenden Arbeit. AASA und PASA entwickeln sich altersabhängig nach Abschluss der Adoleszenz weiter. 
Eine Zunahme der Winkelgröße scheint mit hoher Wahrscheinlichkeit vorzuliegen. Dass G3 einen größeren AASA aufweist als G4, könnte an den Gruppengrößen liegen. G3 weißt mit 668 Hüften deutlich mehr Untersuchungen auf als G4 (n=128).

Eine Kollektiveinteilung in Altersquartile wäre ebenfalls denkbar gewesen und hätte zu ausgeglichenen Gruppengrößen geführt, hätte aber auch mögliche altersabhängigen Unterschiede verschleiern können.

Als denkbare Ursache für die Veränderungen des anterioren und posterioren acetabulären Sektorwinkels im Alter, kann die im Alter abnehmende Muskelmasse diskutiert werden. Damit stiege das Risiko einer Instabilität des Hüftgelenks, wodurch es zu einer vermehrten dorsalen Überdachung des Femurkopfes käme und somit eine größere knochengeführte Stabilität im Hüftgelenk erreicht würde. Gleichzeitig könnte diese Veränderung auch durch unterschiedlich einwirkende Krafteinleitungen oder degenerative Prozesse verursacht sein.

\subsubsection{AI}

Die Auswertungen erbrachten auch für den AI signifikante Messunterschiede zwischen den vier Altersgruppen $(p<0,0001)$. Es konnte eine Abnahme der Winkelgröße mit ansteigendem Alter festgestellt werden. In G1 betrug der AI 2, $8^{\circ}$, in G2 $0,1^{\circ}$, in G3 $-0,9^{\circ}$ und in G4 -0, $7^{\circ}$. Die älteste Gruppe zeigte einen leicht größeren Mittelwert als die nächst jüngere.

Auch Miyasaka et al. (2017) untersuchten den AI. Sowohl bei den männlichen Patienten, als auch bei den weiblichen konnten signifikante altersabhängige Unterschiede festgestellt werden (jeweils $\mathrm{p}<0,001$ ). Ebenso wie im Kollektiv der vorliegenden Arbeit wurde eine altersabhängige Abnahme des AI bei Männern und Frauen festgestellt. So ergaben sich Mittelwerte von 6,7 $\left(<50\right.$ Jahre) und 2,8 $8^{\circ}\left(>50\right.$ Jahre) bei den Männern sowie von $7,2^{\circ}(<50$ Jahre) und $3,3^{\circ}$ ( $>50$ Jahre) bei den Frauen.

Auch wenn in den Auswertungen der vorliegenden Arbeit G4 einen leicht größeren AI aufwies als G3, ist, auch unter Berücksichtigung der Ergebnisse von Miyasaka et al. (2017), von einer Altersabhängigkeit des AI auszugehen. Eine mögliche Verzerrung durch die Gruppengröße wurde bereits unter 4.2 .3 diskutiert.

Diese hier vorgestellten Werte sollten zukünftig als Referenz für weitere Studien verwendet werden. Besonders beachtet werden sollte dies vor dem Hintergrund, dass der AI bislang unzureichend beschrieben ist. 


\subsubsection{SW}

Beim SW konnten ebenfalls signifikante altersabhängige Messunterschiede festgestellt werden $(\mathrm{p}<0,0001)$. Dabei nahmen die Mittelwerte von der jüngeren zur nächstälteren Altersgruppe ab (G1: 40,2 $\left.2^{\circ} \mathrm{G} 2: 37,0^{\circ} ; \mathrm{G} 3: 36,3^{\circ} ; \mathrm{G} 4: 35,5^{\circ}\right)$.

Keine der beiden Vergleichsstudien untersuchte den Einfluss des Alters auf den SW. Die Analyse des SW in Abhängigkeit vom Alter erfolgte somit erstmalig. Die Feststellung, dass sich die physiomorphologische Entwicklung des SW auch nach Abschluss der Adoleszenz fortsetzt ist neu und von besonderer Bedeutung.

Die Ursache ist analog zur Diskussion des LCEs zu sehen, da der SW, wenn auch in messtechnisch anderer Form, ebenfalls das Ausmaß der kraniolateralen Überdachung des Hüftkopfes durch die Pfanne beschreibt.

Der SW kann unter anderem zur Referenzierung für die Pfanneninklination genutzt werden (Nishii et al. 2004). Werden die durch diese Arbeit nachgewiesenen Ergebnisse berücksichtig, sollten zukünftige Studien untersuchen, ob bei Patienten höheren Alters eventuell das Pfannenimplantat mit einer geringeren Inklination als $45^{\circ} \mathrm{zu}$ positionieren ist, um eine bessere physiologische Nachbildung der anatomischen Pfanne zu erreichen und somit möglicherweise auch eine bessere Funktion, als höheren Luxationsschutz, zu erzielen.

\subsubsection{FNV}

Für die FNV konnten keine signifikanten altersabhängigen Messunterschiede festgestellt werden ( $p=0,85)$. Die Mittelwerte der einzelnen Altersgruppen betrugen $11,4^{\circ}(\mathrm{G} 1), 11,8^{\circ}(\mathrm{G} 2)$, $11,7^{\circ}(\mathrm{G} 3)$ und $12,3^{\circ}(\mathrm{G} 4)$.

Vergleichswerte liefert die Studie von Jiang et al. (2015), wobei auch diese Autoren keine signifikanten Messunterschiede zwischen den Patienten unter bzw. über 60 Jahren feststellen konnten. Deren jüngere Altersgruppe wies im Mittel eine FNV von 10,54 auf, die ältere eine von $10,66^{\circ}$.

Anhand dieser Ergebnisse muss der Schluss gezogen werden, dass keine Altersabhängigkeit der physiomorphologischen Entwicklung der FNV besteht. Die Messwerte sollten zukünftig als Referenz für weitere Studien herangezogen werden, die dieses bekräftigen oder eventuell widerlegen.

\subsubsection{CCD}

Für den CCD konnten signifikante Messunterschiede zwischen den vier Altersgruppen gefunden werden $(\mathrm{p}<0,0001)$. 
Dabei nahm die Winkelgröße von der jüngeren Altersgruppe zur nächst älteren ab. Die Patienten mit einem Lebensalter von weniger als 40 Jahren wiesen im Mittel einen CCD von $131,4^{\circ}$ auf. G2 zeigte einen Winkel von $131,3^{\circ}$, G3 von $129,4^{\circ}$ und G4 von $128,9^{\circ}$.

Auch Jiang et al. konnten einen signifikanten Altersunterschied nachweisen. Die untersuchten Patienten wiesen Mittelwerte von 133,97 (<60 Jahre) und 132,42 ${ }^{\circ}$ (>60 Jahre) auf. Ebenso wie in der vorliegenden Arbeit nahm der CCD in der Winkelgröße mit zunehmendem Alter ab.

Diese Erkenntnis ist hochinteressant. Daraus resultiert, dass die physiomorphologische Entwicklung des CCDs nach Abschluss der Adoleszenz weiter fortschreitet. Die kindliche Entwicklung dieses Parameters ist sehr gut untersucht. So sinkt der CCD von ca. $150^{\circ}$ bei Geburt auf einen Winkel von ca. $120^{\circ}$ nach Abschluss der Adoleszenz (Hasler und Hefti 2015). Da nach der Definition von Tönnis und Heinecke (1999) ein CCD-Winkel von $120^{\circ}$ bereits einer pathologischen Coxa vara entspräche (physiologischer Referenzbereich zwischen 125 und $140^{\circ}$ ) ist die Angabe von ca. $120^{\circ}$ als zu ungenau einzustufen. Interessanterweise scheint sich die Entwicklung einer Coxa vara in der adulten Lebensphase physiologisch tatsächlich fortzusetzten. Erstmalig kann diese Beobachtung durch die vorliegende Studie mit großer Fallzahl und dreidimensionalen Messungen anhand von vier Altersgruppen nachgewiesen werden.

Diese Erkenntnis sollte zukünftig als wichtige Referenz für die Diagnostik und Therapie von Hüftpathologien berücksichtig werden. Sowohl in der hüftgelenkerhaltenden (Platten-, Schrauben- und Marknagelosteosynthesen), als auch in der hüftgelenkersetzenden Chirurgie (Endoprothesen) sollte bei der Wahl des richtigen Implantatdesigns diese morphologische Besonderheit Berücksichtigung finden.

Eine mögliche Ursache für die stetige Abnahme der Winkelgröße von Geburt an, könnte der aufrechte Gang sein. Erst in einem Alter von 3 Jahren sinkt der initiale CCD von $150^{\circ}$ bei Geburt auf $148^{\circ}$ (Hasler und Hefti 2015). Nach den Meilensteinen der kindlichen Entwicklung ist sicheres aufrechtes Laufen in einem Alter von 2 Jahren physiologisch (Hellstern 2012; Hassler und Hefti 2015). Das würde erklären warum der CCD nicht bereits früher sinkt. Die Ätiopathogenese könnte von der axialen Belastung, die beim aufrechten Laufen auf den Schenkelhals wirkt, herrühren.

Diese Überlegungen sollten durch weitere Studien untersucht werden. Die vorliegende Arbeit sollte als Referenz dienen und könnte einen wichtigen Grundstein für das Verständnis der physiomorphologischen Entwicklung des CCDs geliefert haben. 


\subsubsection{A $\alpha A$ und $S \alpha A$}

Die Auswertungen ergaben signifikante Altersunterschiede für den $A \alpha A(p<0,0001)$, jedoch nicht für den $\mathrm{S} \alpha \mathrm{A}(\mathrm{p}=0,21)$. Für den anterioren Winkel wurde in $\mathrm{G} 1$ ein Mittelwert von 38,9 gemessen. In G2 betrug dieser $41,9^{\circ}$, in G3 $42,2^{\circ}$ und in $\mathrm{G} 439,3^{\circ}$. Von G1 bis G3 steigt die Winkelgröße an. G4 weist einen kleineren Mittelwert auf als G2 und G3. Ein inverses Bild zeigt $\operatorname{der} \mathrm{S} \alpha \mathrm{A}\left(\mathrm{G} 1: 47,0^{\circ} ; \mathrm{G} 2: 46,0^{\circ} ; \mathrm{G} 3: 45,7^{\circ} ; \mathrm{G} 4: 46,2^{\circ}\right)$. Die Winkelgröße fällt von G1 bis G3, aber G4 präsentiert einen größeren Mittelwert als G2 und G3.

Eine mögliche Ursache könnte in der Gruppengröße liegen, was bereits ausführlich diskutiert wurde. Allerdings wäre auch ein nicht linearer Entwicklungsverlauf der $\alpha$-Winkel denkbar. Das kann anhand der Ergebnisse so nicht abschließend geklärt werden. Deswegen sollten weitere Studien durchgeführt werden, die sich an dieser Arbeit orientieren. Eine Altersabhängigkeit kann zwar aufgrund von fehlender Signifikanz nicht für den $\mathrm{S} \alpha \mathrm{A}$ beschrieben werden, für den AaA jedoch schon. Deswegen sollten die Ergebnisse zumindest auf deskriptiver Basis zukünftig als Referenzwerte verwendet werden. Insbesondere weil aktuell keine Vergleichsstudien vorliegen, die den $\mathrm{A} \alpha \mathrm{A}$ sowie den $\mathrm{S} \alpha \mathrm{A}$ in unterschiedlichen Altersgruppen untersucht haben. Eine solche Betrachtung erfolgt durch die vorliegende Arbeit erstmalig.

Da beide $\alpha$-Winkel eine divergente Entwicklung zeigten, fällt es schwer die Verknüpfung mit einem ätiologischen Zusammenhang herzustellen. Möglicherweise ergeben sich durch weitere Studien neue Hinweise, die dieses Vorhaben erleichtern. Daher ist die Durchführung solcher Untersuchungen sehr wünschenswert.

\subsubsection{AO, FO und GO}

Für alle drei Offset-Parameter konnten signifikante Messunterschiede festgestellt werden ( $\mathrm{p}<0,0001)$. Das AO präsentierte Mittelwerte von 31,2 mm (G1), 32,0 mm (G2), 32,3 mm (G3) und 30,4 mm (G4). G4 wies damit das kleinste AO aller Altersgruppen auf, obwohl die Mittelwerte von G1 zu G3 anstiegen. Für das FO wurde in G1 ein Mittelwert von 37,6 mm gefunden. In G2 betrug dieser 39,0 mm, in G3 41,2 mm und in G4 41,6 mm. Das FO stieg von der jüngeren Altersgruppe zu der nächst älteren an. Beim GO ergaben sich Mittelwerte von 68,7 mm (G1), 71,0 mm (G2), 73,5 mm (G3) sowie 71,9 mm (G4). Auch hier zeigte sich ein Wertanstieg von G1 bis G3. G4 wies allerdings ein kleineres GO auf als G3. Da das GO die Summe aus AO und FO ist, geht diese Beobachtung auf den in Relation kleinen Mittelwert des AO in G4 zurück. Als mögliche Ursache kommt abermals die Gruppengröße in Betracht.

Keine der beiden Referenzstudien beschäftigt sich mit der Untersuchung einer möglichen Altersabhängigkeit der Offset-Parameter. 
Somit erfolgte eine solche Betrachtung in der vorliegenden Studie erstmalig. Weitere Studien zur altersabhängigen Entwicklung der Offset-Parameter sind nicht bekannt. Allein aus diesem Grund sollten die Messwerte zukünftig als Referenz dienen.

Zusammenfassend muss festgehalten werden, dass die Offset-Parameter eine Altersabhängigkeit aufzuweisen scheinen. Darüber hinaus erscheint die chirurgische Rekonstruktion des physiologischen Offsets durch korrekte Implantatauswahl und Implantatpositionierung für das klinische Outcome eine wichtige Rolle zu spielen. Daher haben die dargestellten Ergebnisse auch für die chirurgische Therapie von Hüftpathologien eine besondere Bedeutung.

Dass die Rekonstruktion des physiologischen FO von enormer Bedeutung für das klinische Outcome ist, ist durch aktuelle Studien belegt (Amstutz et al. 2004; Charles et al. 2005); Confalonieri et al. 2008). So resultiert aus einem femoralen Offsetverlust von mehr als $5 \mathrm{~mm}$ nach Implantation einer Hüft-Totalendoprothese eine deutliche Krafteinschränkung der Abduktoren (Mahmood et al. 2016). Ji et al. untersuchten 2017 den postoperativen Outcome hinsichtlich der Rekonstruktion des FOs nach Implantation von bipolaren Hemiprothesen. Dabei orientierten sie sich an zwei anerkannten Scores zur Einschätzung der Belastbarkeit bzw. der Einschränkungen im Alltag, dem Harris Hip Score (Harris 1969) und einem modifizierten Barthel Index (Mahoney und Barthel 1965). Die Autoren zeigten, dass in 23\% der eingeschlossenen Operationen das FO nicht rekonstruiert wurde. Genau für diese Implantationen ergaben sich postoperativ signifikant schlechtere Score-Ergebnisse, als für die Eingriffe, in denen das FO rekonstruiert wurde.

\subsubsection{0 ØA, ØF und F/A}

Für den ØA $(p=0,0006)$, den $\varnothing F(p<0,0001)$ und das F/A $(p<0,0001)$ konnten jeweils signifikante Messunterschiede zwischen den vier Altersgruppen festgestellt werden. Der ØА wies einen Mittelwert von 58,1 mm in G1 auf. In G2 waren es 57,8 mm, in G3 59,1 mm und in G4 57,9 mm. Der ØF präsentierte von G1 bis G3 einen zunehmenden Durchmesser (G1: 46,2 mm; G2: 47,3 mm; G3: 48,4 mm). G4 (47,3 mm) wies jedoch einen kleineren Mittelwert auf als G3. Das Verhältnis aus beiden Durchmessern gibt das F/A wieder. In der jüngsten Altersgruppe ergab sich ein Mittelwert von 79,6\%. In den anderen drei wurde jeweils ein F/A von $82,0 \%$ ermittelt.

Keine der Referenzstudien beschäftigt sich mit der Untersuchung einer Altersabhängigkeit des $\varnothing \mathrm{A}$, des ØF oder des F/A. 
Es sind auch keine weiteren Studien bekannt, die eine derartige Analyse durchführten. Eine solche Untersuchung erfolgte durch die vorliegende Arbeit erstmalig.

Wird das F/A betrachtet, kann festgestellt werden, dass der prozentuale Anteil mit steigendem Alter zunimmt. Es konnten zwar signifikante Messunterschiede zwischen den vier Gruppen für den ØA festgestellt werden, jedoch keine gruppenübergreifende Abnahme oder Zunahme des Mittelwertes. Für den ØF zeigte sich wiederum ein Anstieg von G1 bis G3. Der kleinere Wert in G4 könnte auf die Gruppengröße zurückzuführen sein. Diese Besonderheit wurde bereits diskutiert.

Werden die drei Parameter einzeln betrachtet, fällt es schwer konkrete Entwicklungsmuster und eine mögliche Ursache zu finden. In der Zusammenschau der Parameter ist jedoch festzuhalten, dass physiomorphologische Veränderungen im Zuge des Alterungsprozesses eher beim Hüftkopfdurchmesser zu finden sind, als auf Seiten des Acetabulumdurchmessers. Um eine bessere Einordnung dieser Parameter zu erhalten, sind allerdings weitere Studien notwendig.

\subsubsection{Arthrosegrad}

Zwischen den vier Altersgruppen konnten signifikante Messunterschiede für den Arthrosegrad festgestellt werden $(\mathrm{p}<0,0001)$. In der jüngsten Altersgruppe fand sich ein KL 0 mit einem prozentualen Anteil von 37,2\%, ein KL 1 mit 36,1\% und ein KL 2 mit 26,7\%. KL 3 und KL 4 wurden in G1 nicht gefunden. In G2 (KL 0: 10\%; KL 1: 37,6\%, KL 2: 49,2\%; KL 3: 2,4\%; KL 4: 0,8\%) und in G3 (KL 0: 8,4\%; KL 1: 31,4\%; KL 2: 52,8\%; KL 3: 6,6\%; KL 4: 0,8\%) wurden positive Messungen in jedem Arthrosestadium gefunden. G4 präsentierte keinen KL 0 . Ein KL 1 zeigte sich in 23,4\% der Untersuchungen, ein KL 2 in 53,9\%, ein KL 3 in 18,8\% und ein $\mathrm{KL} 4$ in $3,9 \%$.

Wie zu erwarten war, zeigt sich, dass der radiologische Arthrosegrad mit zunehmendem Alter ansteigt.

Wie bereits in 4.1.11 diskutiert, wurde der Arthrosegrad in dieser Studie aber primär erhoben, um nachzuweisen, dass das untersuchte Kollektiv primär nicht hochgradig hüfterkrankt ist. Ausgehend davon, dass von einer manifesten Arthrose ab einem KL 3 gesprochen werden kann, zeigt sich ein relativ geringer Anteil solcher Messungen. In G4 finden sich gruppenbezogen ca. 20\% manifeste Arthrosen. Wird die relativ kleine Gruppengröße ( $n=128)$ berücksichtigt, wird der geringe Anteil ersichtlich (26 betroffene Patienten). Im Gesamtkollektiv konnten lediglich 6,3\% der untersuchten Hüften detektiert werden, die einen KL 3 oder 4 aufwiesen (45 betroffene Patienten von insgesamt 712 untersuchten). 


\subsubsection{Schlussfolgerungen}

Außer für die FNV und den SaA konnten für alle anderen Parameter altersabhängige signifikante Messunterschiede festgestellt werden. Aus dem Vorhergehenden wird ersichtlich, dass die physiomorphologische Entwicklung des Hüftgelenks nach dem Abschluss der Adoleszenz nicht beendet ist, sondern sich kontinuierlich über die adulte Lebensphase hinweg fortsetzt.

Diese Erkenntnis ist in dieser Form bislang nicht publiziert und somit neu.

Besonders interessant sind die Auswertungen des CCDs. Möglicherweise ist der aufrechte Gang und die damit verbundene axiale Belastung des Schenkelhalses für die Abnahme der Winkelgröße mit zunehmendem Alter von Geburt an verantwortlich.

Die Messwerte sollten zukünftig als Referenzwerte genutzt werden. Außerdem müssen weitere Studien durchgeführt werden, um die ätiologischen Zusammenhänge näher zu beleuchten. Die diskutierten Punkte sollten außerdem in der klinischen Diagnostik und Therapie von Hüftpathologien berücksichtigt werden.

\subsection{Einfluss des Geschlechts auf die Morphologie des Hüftgelenks}

Unterschiede in der Anatomie des Beckens von Männern und von Frauen sind bekannt und beschrieben. So ist das weibliche Becken flacher und weiter als das männliche. Es ergeben sich zwei unterschiedliche Formen. Zum einen existiert die „Kartenherzform“, durch das „Vorspringen des Promontoriums“ beim Mann, zum anderen die „Ellipsenform“ bei der Frau. Außerdem ist der Schambeinwinkel des weiblichen Beckens größer (Drenckhahn und Waschke 2014). Allein diese Tatsachen legen die Vermutung nahe, dass auch Unterschiede in der Morphologie des Hüftgelenks zwischen Mann und Frau bestehen.

Als aussagekräftige Vergleichsstudien könnten abermals die Untersuchungen von Jiang et al. (2015, n=466), Miyasaka et al. (2017, $n=120)$ und Maheshwari et al. (2010, n=172) herangezogen werden. Außerdem beschäftigten sich auch Mayes et al. (2017, n=66), Hartel et al. (2016, n=1070), Hatem et al. (2017, n=150) und Suzuki et al. (2017, n=120) mit einem Vergleich der Morphologie zwischen männlichen und weiblichen Hüften. Tohtz et al. (2010, $\mathrm{n}=168)$ und Preininger et al. (2012, $\mathrm{n}=100)$ untersuchten gezielt mögliche Unterschiede des FOs zwischen Männern und Frauen. 


\subsubsection{LCE}

Für den LCE konnten signifikante $(\mathrm{p}=0,02)$ Messunterschiede festgestellt werden. Die Männer wiesen im Mittel einen Wert von 40,5 auf und die Frauen einen von 41, $8^{\circ}$. Auch Miyaska et al. (2017) und Suzuki et al. (2017) untersuchten die Geschlechtsabhängigkeit des LCE. Mayes et al. (2017) erhoben ebenfalls Messwerte vom LCE bei Männern und Frauen, verglichen jedoch nicht geschlechtsabhängig, sondern sportspezifisch. In Ihrer Studie sollten Unterschiede in der Hüftmorphologie von Tänzern und anderen Athleten herausgestellt werden. Die erhobenen Werte könnten trotzdem auf deskriptiver Basis als Referenz dienen. Mayes et al. (2017) fanden in den weiblichen Hüften in beiden Gruppen einen LCE von 28,0 $0^{\circ}$ Bei den Männern zeigten sich Mittelwerte von 30,9 (Tänzer) sowie von 32,0 (Athleten).

Miyasaka et al. (2017) konnten keinen signifikanten Unterschied zwischen dem LCE von Frauen und Männern feststellen ( $\mathrm{p}=0,424)$. Die Autoren präsentierten Mittelwerte von $32,5^{\circ}$ für Männer und $31,6^{\circ}$ für Frauen. Auch Suzuki et al. (2017) konnten keine signifikanten geschlechtsabhängigen Unterschiede feststellen (Männer: 31,4º, Frauen 29, $6^{\circ}$ ).

Auffällig ist, dass alle drei Vergleichsstudien größere Mittelwerte in den männlichen Hüftgelenken fanden.

Die vorliegende Arbeit zeigte bei weiblichen Hüftgelenken einen größeren LCE. Diese Beobachtung ist insofern erstaunlich, da eigentlich das weibliche Geschlecht ein höheres Risiko besitzt, ein dysplastisches Hüftgelenk auszubilden, als das männliche (Amstutz und Le Duff 2019). Darüber hinaus ist festzustellen, dass bei Betrachtung der in der vorliegenden Arbeit erhobenen Daten, sowohl für das weibliche als auch das männliche Geschlecht, ein um ca. $10^{\circ}$ größerer LCE-Winkel im Vergleich zu den Mittelwerten der Referenzstudien gemessen wurde. Eine Erklärung hierfür liegt nicht vor. Es sollte allerdings berücksichtigt werden, dass alle drei Vergleichsstudien nur sehr kleine Fallzahlen untersucht haben.

\subsubsection{AcetAVa}

Die Mittelwerte der AcetAVa zwischen männlichen und weiblichen Hüftgelenken unterschieden sich signifikant ( $\mathrm{p}<0,0001$ ). Die Männer präsentierten einen Winkel von 19,2 , die Frauen von 23, $1^{\circ}$. Sowohl Mayes et al. (2017), Miyasaka et al. (2017), Hatem et al. (2017) als auch Maheshwari et al. (2010) und Jiang et al. (2015) untersuchten ebenfalls geschlechtsspezifisch die AcetAVa.Mayes et al. (2017) wiesen Mittelwerte von 14,7 (Tänzer) bzw. $18,0^{\circ}$ (Athleten) in den weiblichen Hüften nach und Mittelwerte von $12,1^{\circ}$ (Tänzer) bzw. $15,9^{\circ}$ (Athleten) in den männlichen. 
Auch in der Studie von Miyaska et al. (2017) wurden größere Werte für die AcetAVa in den femininen Hüftgelenken (Männer: 17,1, Frauen: 19,7º gemessen. Ebenso waren die geschlechtsabhängigen Mittelwerte signifikant unterschiedlich $(\mathrm{p}=0,001)$.

Hatem et al. (2017) präsentierten ähnliche Ergebnisse. In ihrem Kollektiv betrug die AcetAVa der Frauen im Mittel 21,23 und die der Männer16,3 $(\mathrm{p}<0,001)$.

Die Ergebnisse von Maheshwari et al. (2010) $(\mathrm{p}=0,01)$ und Jiang et al. $(2015)(\mathrm{p}<0,001)$ fügen sich in dieses Bild ein. Beide Studien zeigten signifikante geschlechtsabhängige Unterschiede für die AcetAVa. Maheshwari et al. fanden in den Hüftgelenken der Männer Mittelwerte von $17,3^{\circ}$, in denen der Frauen von 20,8 . Jiang et al. (2015) konnten eine AcetAVa von $18,27^{\circ}$ in dem männlichen sowie von $20,44^{\circ}$ in den weiblichen Hüftgelenken nachweisen.

Sämtliche Studien, einschließlich der vorliegenden Arbeit, legen den Schluss nahe, dass eine Geschlechtsabhängigkeit der AcetAVa existiert und diese physiologisch bei den Frauen einen größeren Winkel aufweist als bei den Männern.

\subsubsection{AASA und PASA}

Auch die Mittelwerte für den AASA $(p=0,002)$ und den PASA $(p<0,0001)$ zeigten zwischen den Geschlechtern signifikante Unterschiede. Bei den Männern wurden Mittelwerte von 66,5 (AASA) sowie von $104,8^{\circ}$ (PASA) nachgewiesen. In den weiblichen Hüftgelenken betrug der AASA im Mittel $64,9^{\circ}$ und der PASA $110,7^{\circ}$. Daraus folgt, dass die männlichen Patienten im Vergleich zu den weiblichen eine vermehrte anteriore Überdachung aufweisen, aber eine verminderte posteriore.

AASA und PASA wurden auch von Miyasaka et al. (2017) und Suzuki et al. (2017) untersucht. Die erstgenannte Autorengruppe fand ebenfalls einen größeren AASA und kleineren PASA in den männlichen Hüftgelenken im Vergleich zu den weiblichen. Die Mittelwerte betrugen für den anterioren Winkel $61,2^{\circ}$ (Männer) bzw. 57,1 $1^{\circ}$ (Frauen) und für den posterioren 94,5 (Männer) bzw. 96,8 (Frauen). Miyasaka et al. (2017) konnten für beide Parameter signifikante Messunterschiede nachweisen (AASA: $p<0,001$; PASA: $p=0,053$ ).

In diese Darstellung passen ebenfalls die Ergebnisse von Suzuki et al., welche auch signifikante Geschlechtsabhängigkeiten des AASA und PASA finden konnten. Auch in dieser Studie wiesen die Männer einen größeren AASA $\left(59,1^{\circ}\right)$ und kleineren PASA $\left(97,7^{\circ}\right)$ als die Frauen auf (AASA: $55,9^{\circ}$; PASA: $100,8^{\circ}$ ).

Alle drei Studien legen den Schluss nahe, dass eine Geschlechtsabhängigkeit der hüftmorphologischen Parameter AASA und PASA vorliegt. 
Wie bereits unter 4.1.2 bzw. 4.1.3 diskutiert, könnte die vermehrte dorsale Überdachung eine physiomorphologische Entwicklungsfolge der erhöhten AcetAVa (in der vorliegenden Arbeit ebenfalls in weiblichen Hüften größer als in den männlichen) sein. Möglicherweise fungiert die vermehrte Überdachung als Luxationsschutz bei eher geringer ausgebildeter weiblicher Muskulatur (Suetta et al. 2019).

Diese Ausführungen sind sehr interessant und es müssen weitere Studien durchgeführt werden, um die ätiologischen Zusammenhänge besser erfassen sowie belegen zu können.

Auch in der Endoprothetik sollten diese Überlegungen berücksichtigt werden. Es ist wichtig, ein entsprechendes Pfannendesign und die entsprechende Pfannenpositionierung geschlechtsabhängig anzupassen.

\subsubsection{AI}

Die Auswertungen ergaben keine signifikanten Messunterschiede für den AI $(p=0,12)$. In den männlichen Hüften wurde ein Winkel von $-0,1^{\circ}$ gemessen, in den weiblichen einer von $0,4^{\circ}$. Lediglich Miyasaka et al. (2017) untersuchten diesen Parameter ebenfalls. Die Autoren konnten auch keinen signifikanten Unterschied zwischen dem männlichen und weiblichen AI finden $(p=0,448)$. Sie fanden analog zur vorliegenden Arbeit einen größeres AI unter den Frauen $\left(5,3^{\circ}\right)$ als unter den Männern $\left(4,7^{\circ}\right)$. Der absolute Wertunterschied zwischen beiden Studien betrug ca. $5^{\circ}$.

Aufgrund fehlender Signifikanz und dem Nichtvorhandensein weiterer Referenzstudien lässt sich eine Geschlechtsabhängigkeit des AI aktuell nicht nachweisen. Hier zeigt sich, dass weiterhin großer Forschungsbedarf auf dem Gebiet der Hüftmorphologie besteht. Da die Werte der vorliegenden Arbeit erstmalig in einem großen Patientenkollektiv erhoben wurden (Miyasaka et al. [2017] $n=120$ ), sollten sie auf deskriptiver Basis für weitere Untersuchungen als Referenz berücksichtigt werden.

\subsubsection{SW}

In den weiblichen Hüftgelenken wurden mit $37,6^{\circ}$ für den SW signifikant $(p=0,004)$ größere Mittelwerte gefunden als in den männlichen $\left(36,8^{\circ}\right)$. Aktuell liegen keine Vergleichsstudien vor, in denen eine mögliche Geschlechtsabhängigkeit des SW untersucht wurde. Die hier dargestellten Ergebnisse wurden in diesem Sinne erstmalig erhoben. Schon aus diesem Grund sollten die Auswertungen zukünftig als Referenz für weitere Studien dienen.

Eine mögliche Begründung könnte abermals die bereits mehrfach diskutierte Theorie des morphologischen Luxationschutzes liefern. 
Unter der Annahme der im Durchschnitt physiologisch schlechter ausgeprägten Muskulatur der Frauen, gegenüber der der Männer, könnte eine morphologische Entwicklung, im Sinne eines Ausgleiches des dadurch relativ erhöhen Luxationsrisikos, erfolgt sein. Um diese Theorie zu bekräftigen, bedarf es weiterer Studien, die sich mit den Zusammenhängen zwischen Hüftmorphologie und Hüftgelenksfunktion befassen.

Dennoch sollten die Überlegungen auch klinisch berücksichtigt werden. Erneut muss auf das Spezialgebiet Endoprothetik verwiesen werden. Der SW, in seiner Funktion als Indikator für die Ausrichtung des Acetabulums in der Koronarebene, sollte für die Inklination der Pfannenpositionierung geschlechtsabhängig berücksichtig werden.

\subsubsection{FNV}

Die Ergebnisse der vorliegenden Arbeit wiesen einen signifikanten $(p<0,0001)$ geschlechtsspezifischen Unterschied der FNV nach. Dabei zeigten die Frauen im Mittel mit $14,1^{\circ}$ einen größeren Winkel als die Männer mit 10,5 .

Auch Hartel et al. (2016) fanden eine größere FNV in den weiblichen Hüftgelenken (Frauen: $16,4^{\circ}$, Männer: $\left.12,1^{\circ}\right)$. Analog zur vorliegenden Arbeit konnte ein signifikanter $(p=0,0001)$ geschlechtsspezifischer Unterschied für diesen Parameter gefunden werden. Ähnliche Ergebnisse präsentierten auch Jiang et al. (2015, p<0,001). In dem Kollektiv dieser Autoren zeigte sich eine FNV von $14,76^{\circ}$ in den weiblichen Hüftgelenken und von 9,31 ${ }^{\circ}$ in den männlichen. Anhand dieser Darstellung lässt sich ableiten, dass das Vorliegen eines geschlechtsspezifischen Unterschieds der FNV sehr wahrscheinlich ist.

Wieder muss festgehalten werden, dass der größere Mittelwert unter den Frauen gefunden wurde. Möglicherweise spielt auch für die FNV, die physiologisch durchschnittlich schlechter ausgeprägte Muskulatur der Frau eine Rolle. Weitere Studien sollten sich mit dieser Theorie befassen und Untersuchungen anstellen. Die vorliegende Arbeit stellt dafür eine sehr gute Referenz dar.

Auch im Falle der FNV muss über das Prothesendesign in der Hüftendoprothetik nachgedacht werden. Möglicherweise würden geschlechtsspezifische Implantate und eine geschlechtsspezifische Ausrichtung der Implantate einen besseren funktionellen postoperativen Outcome ermöglichen. Beispielsweise könnte in der präoperativen Planung die FNV bestimmt werden und später der Prothesenschaft anhand der Planungswerte präzise ausgerichtet werden.

Zukünftig sollte im Bereich der Prothesenimplantation berücksichtig werden, dass abhängig vom Geschlecht eine unterschiedliche FNV vorliegt. 


\subsubsection{CCD}

Beim CCD konnten signifikante geschlechtsspezifische Messunterschiede festgestellt werden $(p=0,014)$. Im Mittel wurde in den männlichen Hüftgelenken mit 130,6 ein größerer CCD-Winkel gefunden, als in den weiblichen Hüftgelenken mit 129, $4^{\circ}$.

Mayes et al. (2017), Hartel et al. (2016) und Jiang et al. (2015) untersuchten den CCD ebenfalls. Die erstgenannte Autorengruppe fand größere Winkel unter den Frauen (Tänzerinnen: 135, $4^{\circ}$; Athletinnen: $131,8^{\circ}$ ). Die männlichen Tänzer präsentierten Mittelwerte von 133, $7^{\circ}$ und die Männer aus der Athletengruppe von 129,6 $6^{\circ}$. Hartel et al. (2016) wiesen ebenfalls signifikante Messunterschiede nach $(p=0,007)$. Sie fanden in den weiblichen Hüften $\left(123,0^{\circ}\right)$ größere Mittelwerte, als in den männlichen $\left(121,5^{\circ}\right)$. Jiang et al. (2015) zeigten zwar analog zur vorliegenden Arbeit größere CCD-Werte unter den untersuchten Männern $\left(133,13^{\circ}\right)$, konnten aber keinen signifikanten geschlechtsspezifischen Unterschied nachweisen $(p=0,234)$. Die Frauen im Kollektiv präsentierten im Mittel einen CCD von 132,57.

Zusammenfassend zeigt sich für den geschlechtsspezifischen Mittelwert des CCDs ein heterogenes Bild im Vergleich der Studien. Beide Untersuchungen mit hohen Fallzahlen (Hartel et al. [2016] n=1070; vorliegende Arbeit n=712) konnten signifikante Messunterschiede nachweisen, präsentieren allerdings entgegengesetzte Darstellung des geschlechtsabhängig größeren CCD-Winkels (Hartel et al. [2016] Frauen>Männer; vorliegende Arbeit Männer>Frauen). Mayes et al. (2017) und Jiang et al. (2015) konnten keine signifikanten geschlechtsspezifischen Unterschiede nachweisen. Jiang et al. (2015) fanden jedoch, wie auch in der vorliegenden Arbeit, größere CCD-Werte auf Seiten der Männer.

Aktuell sollte davon ausgegangen werden, dass geschlechtsspezifische Unterschiede des CCDs bestehen. Wird berücksichtigt, dass Hartel et al. von den 1070 Patienten nur die linken Hüften untersuchten, könnte dadurch möglicherweise eine Verzerrung der Messwerte vorliegen.

Da die vorliegende Arbeit sowohl linke, als auch rechte Hüften untersucht, sollten die präsentierten Ergebnisse zukünftig in jedem Fall als Referenz für weitere Studien genutzt werden.

\subsubsection{AaA und $\mathrm{S \alpha A}$}

Signifikante Messunterschiede konnten für den $\mathrm{A} \alpha \mathrm{A}(\mathrm{p}<0,0001)$, nicht jedoch für den $\mathrm{S} \alpha \mathrm{A}$ $(0,15)$, festgestellt werden. Die untersuchten Männer (A $\left.\alpha \mathrm{A}: 42,1^{\circ} ; \mathrm{S} \alpha \mathrm{A}: 46,3^{\circ}\right)$ zeigten im Mittel die größeren $\alpha$-Winkel, als die Frauen (A $\left.\alpha \mathrm{A}: 39,6^{\circ}, \mathrm{S} \alpha \mathrm{A}: 45,6^{\circ}\right)$. 
Lediglich Mayes et al. (2017) beschrieben ebenfalls geschlechtsspezifische Messwerte dieser Winkel. Beim S $\alpha$ A fanden sie ähnliche Ergebnisse. In den männlichen Hüften wurden größere Winkel gemessen (Tänzer: 42, $1^{\circ}$; Athleten: $51,4^{\circ}$ ), als in den weiblichen (Tänzerinnen: 36,6 ${ }^{\circ}$; Athletinnen: $42,8^{\circ}$ ). Die untersuchte Tänzergruppe zeigte außerdem für den A $\alpha$ A dieselbe Verteilung. Bei den Männern (47, $\left.7^{\circ}\right)$ wurden größere Mittelwerte nachgewiesen (Tänzerinnen: $40,2^{\circ}$ ). In der Athletengruppe wiesen jedoch die Frauen (46,5 $5^{\circ}$ den größeren A $\alpha \mathrm{A}$ auf (Männer: $\left.45,5^{\circ}\right)$

Durch den Vergleich mit der Studie von Mayes et al. erscheint ein geschlechtsspezifischer Unterschied beider $\alpha$-Winkel wahrscheinlich. Da die vorliegende Arbeit die deutlich größere Fallzahl aufweist ( $n=712$ gegenüber $n=66)$, sollten die Ergebnisse zukünftig als Referenz verwendet werden.

Da der A $\alpha$ A klinisch für die Detektion des Cam-Impingement genutzt wird, muss zukünftig in der radiologischen Diagnostik berücksichtigt werden, dass geschlechtsspezifische Unterschiede bestehen.

\subsubsection{AO, FO und GO}

Für alle Offset-Parameter konnten signifikante Messunterschiede nachgewiesen werden (jeweils p<0,0001). Die Männer (AO: 33,7 mm; FO: 40,8 mm; GO: 74,5 mm) wiesen im Mittel für jeden der drei Parameter den größeren Wert auf, als die Frauen (AO: 28,2 mm; FO: $38,4 \mathrm{~mm}$; GO: 66,6 mm).

Keine der Referenzstudien beschäftigt sich mit einem geschlechtsspezifischen Vergleich des AOs und des GOs. Eine solche Erhebung erfolgte erstmalig. Allein aus diesem Grund sollten die Ergebnisse zukünftig als Referenz berücksichtig werden. Tohtz et al. (2010) untersuchten eine Geschlechtsabhängigkeit des FOs. Sie konnten einen signifikanten Messunterschied feststellen (Männer: 21,31 mm, Frauen: 24,63 mm). Die Ergebnisse der Autorengruppe unterscheiden sich von denen der vorliegenden Arbeit. Zum einen sind die absoluten Werte um einiges geringer, zum anderen präsentierten die Frauen das größere FO. Ursächlich könnte die geringe Stichprobengröße der Vergleichsstudie gewesen sein ( $n=168)$. Preininger et al. (2012) konnten ebenfalls signifikante geschlechtsspezifische Unterschiede des FOs zwischen Männern und Frauen nachweisen (Männer: 43,6 mm, Frauen: 39,5 mm ( $<<0,001)$. Diese Ergebnisse sind denen der vorliegenden Arbeit ähnlich. Eine weitere Studie die sich mit einer Erhebung des FOs (geschlechtsunabhängig) befasste, zeigte einen Mittelwert von 42,2 mm (Sariali et al. 2009) und liegt damit ebenfalls deutlich über den Werten von Tohtz et al. (2010). 
Aufgrund der deutlich größeren Anzahl an untersuchten Patienten $(n=712)$ sowie den Vergleichsstudien von Preininger et al. (2012, $\mathrm{n}=100)$ und Sariali et al. (2009, $\mathrm{n}=112)$, ist davon auszugehen, dass die Ergebnisse von Tohtz et al. fehlerbehaftet sind. Die Ergebnisse der vorliegenden Arbeit sollten in jedem Fall zukünftig als Referenz berücksichtigt werden.

Als Erklärung für die höheren Werte in den männlichen Hüften scheint die Körpergröße der entscheidende Faktor zu sein. Im Durchschnitt sind Männer größer als Frauen (Bentham et al. 2016). Auch wenn diese Überlegung naheliegend scheint, so ist sie erstmalig für die speziellen morphologischen Parameter anhand von Messwerten belegt wurden.

\subsubsection{0 ØA, ØF und F/A}

Für ØA und ØF konnten signifikante Messunterschiede nachgewiesen werden (jeweils $p<0,0001)$, nicht jedoch für das F/A ( $p=0,80)$. In den männlichen Hüften wurden größere Werte für den ØA (60,6 mm) und den ØF (49,4 mm), als in den weiblichen (ØA: 54,2 mm; ØF: 44,2 mm), festgestellt. Das F/A betrug bei beiden Geschlechtern 81,6\%.

Keine Referenzstudie untersuchte diese drei Parameter. Analog zu den Offset-Messdaten erfolgte eine solche Erhebung erstmalig. Deswegen sollten auch diese Ergebnisse zukünftig als Referenz verwendet werden.

Der geschlechtsspezifische Unterschied des ØA und ØF lässt sich ebenfalls am besten mit der Körpergröße erklären, die bei Männern durchschnittlich größer ist. Interessanterweise liefert das F/A geschlechtsunabhängig dasselbe Messergebnis.

Erstmalig wird diese Überlegung durch Messdaten aus einem großen Kollektiv belegt.

\subsubsection{Arthrosegrad}

Für den Arthrosegrad ergab sich ein signifikanter Messunterschied $(p=0,034)$. In den männlichen Hüften fanden sich 13,3\% mit einem KL 0, 34,7\% mit einem KL 1, 45,4\% mit einem KL 2, 6,1\% mit einem KL 3 und 0,5\% mit einem KL 4. Bei den Frauen lagen 13,3\% der Hüften mit einem KL 0 vor, 30,1\% mit KL 1, 50,8\% mit KL 2, 4,2\% mit KL 3 und 1,7\% mit KL 4. Ein Erklärungsansatz für diese Ergebnisse findet sich nicht und keine der Vergleichsstudien untersucht die Abhängigkeit des Arthrosegrades vom Geschlecht.

\subsubsection{Schlussfolgerungen}

Für alle Messparameter, mit Ausnahme des AI, des S $\alpha$ A und des F/A, konnten signifikante geschlechtsspezifische Unterschiede in der Hüftmorphologie nachgewiesen werden. Viele Referenzstudien beschäftigten sich ebenfalls mit einer ähnlichen Fragestellung wie der Teilaspekt der vorliegenden Arbeit. 
Einige Parameter, beispielsweise der LCE, die AcetAVa oder der AASA bzw. PASA, waren bereits ausführlicher untersucht worden, andere wie z.B. die Offset Parameter überhaupt nicht.

Die vorliegende Arbeit konnte erstmalig anhand eines großen Patientenkollektives nachweisen, dass die Morphologie des Hüftgelenks sich geschlechtsspezifisch unterscheidet. Als möglicher Erklärungsansatz kommt die im Durchschnitt stärker ausgeprägte Muskulatur und durchschnittlich höhere Körpergröße des Mannes gegenüber der der Frau in Frage.

Die dargestellten Ergebnisse sollten zukünftig als Referenzwerte in der Hüftgelenkmorphologie für Diagnostik und Therapie berücksichtig werden.

Diese Erkenntnisse sind insbesondere für den Bereich der Hüftgelenkendoprothetik von Bedeutung.

Ein geschlechtsspezifisches Implantatdesign und eine geschlechtsabhängige Prothesenpositionierung anhand präoperativer morphologischer Messwerte und einem Abgleich mit morphologischen Referenzwerten könnten die postoperativen Ergebnisse zukünftig verbessern.

\subsection{Vergleich mit pathologischen Referenzwerten für die Hüftgelenkmorphologie}

In der aktuellen Literatur finden sich radiologische Referenzwerte, die für die Differenzierung von physiologischen und pathologischen Veränderungen der Hüftgelenkmorphologie verwendet werden. Diese basieren jedoch in großen Teilen auf Studien, die nur ein kleines Studienkollektiv untersucht haben (Mayes et al. 2017; Cooke et al. 2013; Buller et al. 2012; Miyasaka et al. 2017). Mayes et al. (2017) analysierten Hüftgelenke von 66 Patienten, Cooke et al. von 53 Patienten, Buller et al. (2012) von 115 Patienten und Miyasaka et al. (2017) von 120 Patienten.

Demgegenüber stehen Studien mit großer Fallzahl. In diesen wurden jedoch zumeist nur wenig Parameter erhoben (Hartel et al. 2016; Nardo et al. 2015). Erstere werteten zwar 1070 CT-Datensätze aus, erhoben aber lediglich Messwerte für die FNV und den CCD. Nardo et al. (2015) untersuchten zwar ein großes Patientenkollektiv $(n=4140)$, analysierten aber Röntgenbilder und keine CT-Datensätze. In ihrer morphologischen Analyse der Hüfte betrachteten die Autoren nur den LCE und den CCD. Auch andere Vergeleichsstudien basieren nur auf Röntgenbildern und nicht auf CT-Scans (Shi et al. 2010; Cooke et al. 2013). 
Aktuell existiert keine Vergleichsstudie, die ein primär asymptomatisches Patientenkollektiv mit großer Fallzahl analysiert und dabei eine umfangreiche Erfassung radiologischer Parameter für die Hüftgelenkmorphologie mittels moderner CT-Bildgebung aufweist. Somit besitzt die vorliegende Arbeit ein Alleinstellungsmerkmal in der radiologischen Analyse der Hüftgelenkmorphologie.

Ferner findet sich aktuell ebenfalls keine Studie, die das Auftreten von pathologischen Werten in einem radiologisch „hüftgesunden“ bzw. asymptomatischen Kollektiv untersucht. Unter anderem definierten Tönnis und Heinecke 1999 den physiologischen Referenzbereich des CCD-Winkels zwichen $125^{\circ}$ und $140^{\circ}$. Ob Werte außerhalb dieser Grenzen möglicherweise auch in einem radiologisch ,hüftgesunden“ Kollektiv auftreten, wurde bisher nicht untersucht. Zur Validierung des tatsächlichen Vorliegens eines „,hüftgesunden“ Patientenkollektives wurde der Arthrosegrad nach Kellgren/Lawrence bestimmt. Da nur ein geringer Anteil (ca. 6\%) an manifesten radiologischen Arthrosen (KL 3 und 4) nachgewiesen werden konnte, kann von einem radiologisch primär asymptomatischen Kollektiv ausgegangen werden.

Abschließend erscheint es sinnvoll, die Ergebnisse der vorliegenden Arbeit mit den, aus der aktuellen Literatur geltenden, pathologischen Referenzwerten für die Hüftgelenkmorphologie zu vergleichen.

\subsubsection{Coxa vara und Coxa valga}

Schenkelhalsanomalien können die Entstehung einer Coxarthrose triggern. Als präarthrotischer Marker kann der CCD-Winkel herangezogen werden. Insbesondere eine stark ausgeprägte Coxa vara kann für die Entstehung einer Coxarthrose ursächlich sein (Harrasser et al. 2016).

Als physiologischer CCD ist eine Winkelgröße von $125-140^{\circ}$ definiert (Tönnis und Heinecke 1999). Werte unterhalb dieses Referenzbereiches werden als Coxa vara, Werte darüber als Coxa valga bezeichnet.

In dem untersuchten Kollektiv der vorliegenden Arbeit zeigten 33\% der rechten und 17,4\% der linken Hüftgelenke einen $\mathrm{CCD}<125^{\circ}$. Eine Coxa valga wurde weniger häufig gefunden. In den rechten Hüften waren 7,6\% betroffen und in den linken 13,9\%.

Wird berücksichtigt, dass der CCD mit zunehmendem Alter physiologisch auch in der adulten Lebensphase varischer $\mathrm{zu}$ werden scheint (4.2.7; Jiang et al. [2015]), so muss darüber nachgedacht werden, ob der ,pathologische“ Referenzwert eines $\mathrm{CCD}<125^{\circ}$ im höheren Alter nach unten zu korrigieren ist. 
Es sollte allerdings berücksichtigt werden, dass der mittlere CCD in der Altersgruppe über 80 Jahren in der vorliegenden Arbeit bei $128,9^{\circ}$ und damit innerhalb des Referenzbereiches liegt. In der Altersgruppe 60-80 Jahre betrug der Mittelwert des CCD-Winkels 129,4 und in der Altersgruppe 40-60 Jahre 131,3 ${ }^{\circ}$. Basierend auf diesen Ergebnissen sollte ein neuer altersspezifischer Referenzwert ab dem sechzigsten Lebensjahr statt $<125^{\circ}$ auf $<120^{\circ}$ herabgesetzt werden. Dieses würde der nachgewiesenen altersabhängigen Entwicklung des CCD-Winkels gerechter werden.

Vor allem für die klinische Diagnostik sowie eventuelle Operationsindikationsstellungen sind diese Überlegungen sehr wertvoll und sollten zukünftig in der Praxis berücksichtigt werden.

\subsubsection{Coxa retrotorta und Coxa antetorta}

Eine pathologische Torsion des Femurs wird anhand der FNV verifiziert. Das Intervall von 5-25 wird als physiologisch angesehen (Hetsroni et al. 2013).

In der vorliegenden Studie wurden nur wenige Hüftgelenke mit einer FNV $>25^{\circ}(8,6 \%$ linksseitig und 7,2\% rechtsseitig), was der sogenannten Coxa antetorta entspricht, gefunden. Unterhalb des Referenzintervalls fanden sich hingegen deutlich mehr ,pathologische“ Ergebnisse. In ca. 25\% der untersuchten Hüftgelenke (rechts und links) konnte eine FNV $<5^{\circ}$ (Coxa retrotorta) festgestellt werden. Das entspricht einem Viertel des untersuchten Kollektivs. Dieser Anteil erscheint in einem radiologisch „hüftgesunden“ Kollektiv zu hoch, sodass der als pathologisch geltende radiologische Referenzwert kritisch hinterfragt werden sollte.

Diskutabel ist das Vorliegen eines durch Lagerung bedingten Rotationsfehlers im Kniegelenk, wodurch sich der Wert der inferioren Femoral neck version, durch Änderung der Position der Femurkondylen, verändern könnte. Unter der Überlegung, dass sich in einem solchen Fall aber auch Femurkopf und Femurschaft mitbewegen würden, ist diese Fehlerquelle auszuschließen.

Es würde eine Kompensation durch gleichzeitig angepasste Veränderung der superioren Femoral neck version erfolgen. Wird der Anteil an manifesten Retrotorsionstellungen (FNV $\left.<0^{\circ}\right)$ betrachtet, lag dieser bei ca. 10\% aller untersuchten Hüftgelenke vor (9,3\% linksseitig und $11,7 \%$ rechtsseitig).

Daher sollte darüber nachgedacht werden, den unteren Referenzbereich bei einer FNV $<0^{\circ}$ anzusiedeln. Der Begriff Coxa retrotorta würde dann auch einer mathematisch korrekten Definition entsprechen. Dieses würde das Verständnis im klinischen Alltag erleichtern. 


\subsubsection{Cam-Impingement}

Das symptomatische Cam-Impingement gilt als behandlungsbedürftige präarthrotische Deformität und wird anhand des $\mathrm{A} \alpha \mathrm{A}\left(>55^{\circ}\right)$ detektiert (Nötzli et al. 2002). Im untersuchten Kollektiv wiesen 1,4\% der linken Hüften sowie 5,1\% der rechten Hüften einen pathologischen Winkel auf. Dieser geringe Anteil passt zum asymptomatischen Charakter der Studiengruppe.

Kopec et al. untersuchten den AaA 2017 in einem Patientenkollektiv mit einer schmerzpositiven sowie einer schmerznegativen Kontrollgruppe. Dieses umfasste 500 Patienten, wovon 269 eine Schmerzsymptomatik aufwiesen und 231 nicht. In der asymptomatischen Vergleichsgruppe wiesen 20,8\% der Untersuchungen einen pathologischen Winkel auf. Dieser Wert liegt deutlich höher als der der vorliegenden Arbeit. Es sollte jedoch berücksichtig werden, dass die Fallzahl mit n=231 deutlich niedriger liegt (vorliegende Arbeit: $\mathrm{n}=712$ ), was möglicherweise eine Verzerrung bedingen könnte.

Außerdem sollte bedacht werden, dass der $\mathrm{A} \alpha \mathrm{A}$ insbesondere der Früherkennung dient. Über den Winkel wird eine präarthrotische Deformität und keine manifeste Arthrose detektiert. Dementsprechend muss bei einem pathologischen A $\alpha \mathrm{A}$ nicht gleichzeitig eine klinische Beschwerdesymptomatik vorliegen. Vielmehr kann sich diese bei ausbleibender therapeutischer Intervention erst entwickeln.

Zusammenfassend liefern die Ergebnisse der vorliegenden Arbeit keinen Anhalt dafür, dass der pathologische Referenzwert des A AA falsch sein könnte. Daher sollte dieser weiter zur klinischen Früherkennung des Cam-Impingement genutzt werden.

\subsubsection{Dysplasie, Borderline-Dysplasie und acetabuläres Overcoverage (Pincer- Impingement)}

Der LCE wird mit dem Referenzbereich eines Winkels von 25,1-40 ${ }^{\circ}$ als physiologisch betrachtet. Als pathologisch gelten Werte von $<20^{\circ}$ (Dysplasie), 20-25 ${ }^{\circ}$ (Borderline) und $>40^{\circ}$ (Overcoverage bzw. Pincer-Impingement [Lee und Kim 2012]).

Werte, die demnach auf eine Dysplasie oder einen Borderline-Typ schließen lassen würden, waren in dem untersuchten Kollektiv nur in geringer Zahl auffindbar (Dysplasie: 0\% linksseitig und 0,7\% rechtsseitig; Borderline: 0,7\% linksseitig und 3,4\% rechtsseitig). Gegenteilig verhielt sich dies jedoch bei den LCE-Werten $>40^{\circ}$. In den rechten Hüftgelenken zeigten sich mit $42 \%$ der Untersuchungen, Messwerte, die oberhalb des als physiologisch definierten Referenzintervalls lagen. Linksseitig waren es $61,7 \%$. 
Wird berücksichtigt, dass der LCE physiologisch mit zunehmendem Alter anzusteigen scheint (4.2.1), muss hinterfragt werden, ob der Referenzwert $>40^{\circ}$ weiterhin als pathologisch betrachtet werden kann oder nach oben hin korrigiert werden sollte. Klinisch soll anhand eines LCE $>40^{\circ}$ ein Pincer-Impingement erkannt werden, um frühestmöglich therapeutisch intervenieren zu können, sodass letztlich die Entstehung einer Coxarthrose verhütet wird. Bei Patienten in den letzten Lebensjahren ist ohnehin fraglich, ob eine präarthrotische Deformität sich im Zeitraum des verbleibenden Lebens zur manifesten Arthrose entwickelt. Unter Anbetracht des physiomorphologischen Anwachsens des LCE im Alterungsprozess erscheint es sinnvoll, einen neuen und altersspezifischen Referenzwert für eine pathologisch vermehrte Überdachung des Femurkopfes durch das Acetabulum zu definieren. Die vorliegende Arbeit zeigte einen mittleren LCE von 40,6 in der Altersgruppe 40-60 Jahre. Dieser Wert wäre nach dem aktuellen Referenzwert bereits pathologisch. Die Untersuchung erbrachte Mittelwerte von $43,0^{\circ}$ (60-80 Lebensjahre) sowie von $44,0^{\circ}$ (>80 Lebensjahre). Abgeleitet aus diesen Ergebnissen erscheint ein neuer Referenzwert von $>45^{\circ}$ sinnvoll.

Diese Überlegungen sollten zukünftig in der klinischen Diagnostik sowie den damit im Zusammenhang stehenden Therapieentscheidungen berücksichtig werden.

\subsubsection{Pathologische Retro- und Anteversion des Acetabulums}

Die Retro- bzw. Anteversion des Acetabulums wird anhand der AcetAVa bestimmt. Als pathologisch werden Werte $<0^{\circ}$ und $>15^{\circ}$ angesehen (Stem et al. 2006). Eine absolute Retroversionsstellung $\left(<0^{\circ}\right)$ fand sich im gesamten Kollektiv nicht. Ein komplett anders Bild zeigte der Anteil der Untersuchungen mit einer AcetAVa $>15^{\circ}$. Auf der rechten Hüftseite mit $79,2 \%$ und auf der linken mit 79,1\% wurden Werte gemessen, die nach Definition „pathologisch“" wären.

Der Mittelwert aller Untersuchungen ergab eine AcetAVa von 20,5 $5^{\circ}$ Dieser läge somit ebenfalls im pathologischen Bereich. Auch die Vergleichsstudien von Miyasaka et al. (2017, $\left.18,4^{\circ}\right)$ und Hatem et al. $\left(2017,18,6^{\circ}\right)$ präsentierten demnach im Mittel pathologische Werte. Unter Berücksichtigung der altersabhängigen physiomorphologischen Entwicklung der AcetAVa, dem Vorliegen eines asymptomatischen Kollektivs sowie den Ergebnissen der Vergleichsstudien muss der ,pathologische“ Referenzwert von $>15^{\circ}$ als zu niedrig angesehen werden.

Ein neuer Referenzwert von $>25^{\circ}$ erscheint sinnvoll und sollte zukünftig klinisch etabliert werden. Dieses ist für diagnostische sowie therapeutische Entscheidungen von großer Bedeutung. 


\subsubsection{Pfannendysplasie in der Axialebene}

Zur Erfassung der Überdachung des Femurkopfes durch das Acetabulum in der Axialebene werden der AASA und der PASA genutzt. Auch die Referenzwerte für den AASA und den PASA finden sich in aktueller Lehrliteratur wieder (Harrasser et al. 2016).

Ein AASA $<52^{\circ}$ gilt als Dysplasiemarker und fand sich lediglich in 7,4\% der untersuchten Fälle linksseitig bzw. in 4,4\% der Fälle rechtsseitig. Für den PASA gilt ein Winkel $<90^{\circ}$ als pathologisch. In diesen Bereich lagen 10,8\% der linken Hüftgelenke sowie 7,3\% der rechten.

Da sich pathologische Messungen nur in geringen Anteilen in dem untersuchten Kollektiv wiederfinden, erscheinen die Referenzwerte korrekt. Demzufolge sollten diese weiterhin oder zukünftig vermehrt klinisch verwendet werden um frühzeitig die Entstehung einer Arthrose verhindern $\mathrm{zu}$ können. Außerdem sind diese auch für die präoperative Planung endoprothetischer Operationen der Hüfte hilfreich.

\subsubsection{Dysplasie und Overcoverage detektiert durch den SW}

Ein weiterer Parameter, der die Überdachung des Femurkopfes durch das Acetabulum erfasst, ist der SW, welcher in der Koronarebene bestimmt wird. Der physiologische SW liegt in einem Referenzbereich von $38-43^{\circ}$. Werte zwischen $34-37,9^{\circ}$ werden als sogenanntes mildes „Overcoverage“ bezeichnet. Ab einem Winkel $<34^{\circ}$ wird von der schweren Ausprägungsform gesprochen (Tannast et al. 2015).

Die milde Variante lag in dem untersuchten Kollektiv mit 28\% linksseitig und mit 33,4\% rechtsseitig vor. Bei der schweren konnten simultan $27,8 \%$ respektive $22,3 \%$ gemessen werden. Werden beide Gruppen zusammengefasst, weisen über 50\% der untersuchten Hüftgelenke einen „pathologischen“ SW unterhalb des Referenzbereiches auf. Auch dieses erscheint in Bezug auf das radiologisch asymptomatische Kollektiv zu hoch, sodass auch hier die Referenzwerte in Frage gestellt werden müssen. Ein SW $>43^{\circ}$ (Dysplasie) wurde mit einem Anteil von 7,7\% (linke Hüftseite) bzw. 12,9\% (rechte Hüftseite) gefunden. Der Referenzwert erscheint im Vergleich zur Overcoverage-Referenz korrekter.

Bei der Betrachtung der Mittelwertanalyse fällt auf, dass dieser mit 37, $1^{\circ}$ nach den aktuellen Referenzwerten bereits als pathologisch im Sinne eines milden Overcoverage einzuordnen wäre. Genauso verhält es sich mit dem Mittelwert der Altersgruppe 40-60 Lebensjahre. Dieser beträgt $37,0^{\circ}$. Wird berücksichtig, dass der SW physiomorphologisch im Zuge des Alterungsprozesses abnimmt, muss die Korrektheit des ,pathologischen“ Referenzwertes SW $<38^{\circ}$ angezweifelt werden. 
Ein Winkel $<34^{\circ}$ als Referenzwert für ein mildes Overcoverage anstelle eines schweren, erscheint unter Zusammenschau der vorherigen Diskussionen sinnvoll.

Diese Überlegungen sollten zukünftig in der klinischen Diagnostik und daraus resultierenden Therapieplanungen bzw. -entscheidungen berücksichtigt werden.

\subsubsection{Schlussfolgerungen}

In dieser Studie wurde erstmals in einem „hüftgesundem“ Kollektiv morphologische Parameter des Hüftgelenkes in hoher Fallzahl erhoben und mit den in der Literatur publizierten Referenzwerten verglichen.

Dabei zeigte sich das einige Referenzwerte für die Hüftgelenkmorphologie wie z.B. der A $\alpha$ A $>55^{\circ}$ (Detektion des Cam-Impingement) oder der AASA $<52^{\circ}$ bzw. PASA $<20^{\circ}$ (Pfannendysplasie) durchaus als korrekt betrachtet werden können, da diese im untersuchten Kollektiv nur in geringen Teilen auftraten. Es erscheint aus der vorliegenden Arbeit ersichtlich, dass die Werte der altersbedingten physiomorphologischen Entwicklung angepasst werden sollten. Beispielsweise erscheint ein $\mathrm{CCD}<125^{\circ}$ im höheren Alter nicht zwangsläufig pathologisch zu sein. Deswegen sollte über altersspezifische pathologische Referenzwerte nachgedacht werden.

Die vorliegende Arbeit konnte drei Parameter (AcetAVa, LCE und SW) für das Acetabulum und einen Parameter für das proximale Femur (FNV) identifizieren, bei denen in der aktuellen Literatur das physiologische Referenzintervall falsch beschrieben erscheint. Für diese Parameter wurde in der vorliegenden Arbeit ein neues physiologisches Referenzintervall vorgeschlagen.

Weitere Studien sollten die neu vorgeschlagenen physiologischen Referenzintervalle für die AcetAVa, den LCE, den SW und die FNV zukünftig überprüfen. Gleichzeitig sollten die neu vorgeschlagenen Referenzintervalle in der Diagnostik und Therapie von Hüftgelenkmorphologien und -pathologien Berücksichtigung finden.

\subsection{Schwächen der Studie}

Die Schwächen der Studie sind zum einen, dass kein Schmerzscore erhoben wurde und zum anderen keine biometrischen Daten vorliegen. Diese Erhebungen waren im Studiendesign allerdings auch nicht vorgesehen, da die Untersuchungen rein retrospektiver Natur sein sollten. 
Trotzdem muss angemerkt werden, dass beide Aspekte die Aussagekraft der Ergebnisse noch weiter steigern würden. Durch den Schmerzscore hätte zusätzlich zum radiologischen Nachweis eines primär asymptomatischen Patientenkollektivs auch eine klinische Erfassung des Schmerzzustandes vorgelegen.

Ebenso wäre eine Betrachtung der biometrischen Daten interessant gewesen, da sich die Frage stellt, in wie weit sich beispielsweise Körpergröße, Körpergewicht oder der BMI auf die morphologische Entwicklung des Hüftgelenks auswirken.

Ein weiterer Aspekt, der angemerkt werden muss ist, dass die radiologische Vermessung der Patienten im Rahmen dieser retrospektiven Studie nicht nach einem standardisierten Lagerungsschema auf dem Untersuchungstisch erfolgte. Somit ist eine Verkippung des Beckens oder der proximalen Femora möglich und würde entsprechende Fehlmessungen nach sich ziehen. Um diesen Fehler jedoch bestmöglich zu minimieren, wurde für jeden Patienten die Beckenverkippung bestimmt. Es erfolgte eine Messausgleich der lagerungsabhängigen Parameter (Kapitel 2.3), sodass dieser Aspekt die Ergebnisse der Studie unserer Einschätzung nach nicht beeinflusst. 


\section{$5 \quad$ Zusammenfassung}

Das Ziel der vorliegenden Promotionsarbeit war die Erhebung zahlreicher hüftmorphologischer Parameter in einem primär asymptomatischen Patientenkollektiv. Dadurch sollte eine deskriptive Aussage über mögliche Veränderungen der Hüftmorphologie im Erwachsenalter ermöglicht werden. Zudem sollten die erhobenen Werte aus einem großen Patientenkollektiv (1424 Hüftgelenke) mit publizierten Referenzwerten, zumeist aus Studien mit kleinen Fallzahlen, verglichen werden. Anders als bei bisher durchgeführten Studien zur Hüftgelenkmorphologie wurde ein großes asymptomatische Patientenkollektiv retrospektiv mittels computertomographischer Schnittbildgebung analysiert. Hierdurch wurde die Erhebung validerer Werte als bei Studien mit kleiner Fallzahl, symptomatischen Patienten oder nativradiologischer Analyse erwartet.

Basierend auf computertomographischen Angio- und Polytrauma-Scans wurden initial 1000 Patienten (2000 Hüften) vermessen. Im Zuge einer Qualitätskontrolle mussten Messungen exkludiert werden, sodass 712 Patienten (1424 Hüften) abschliessend in die statistische Auswertung eingeschlossen werden konnten. Bei Betrachtung der Datenverteilung zeigte sich ein Übergewicht von CT-Angio-Bildern (80,8\%) im Vergleich zu CT-Polytrauma-Scans $(19,2 \%)$ sowie ein Übergewicht an männlichen Patienten $(66,2 \%)$ im Vergleich zu weiblichen $(33,8 \%)$.

Bei der Analyse der erhobenen Daten ergab sich in Bezug auf die Fragestellungen Folgendes:

Bei der Auswertung, ob die Körperseite einen Einfluss auf die Hüftmorphologie besitzt, zeigten sich für diverse Parameter signifikante Unterschiede. Hypothetisch lässt sich vermuten, dass die morphologischen Differenzen durch die Körperseitendominanz bedingt sind.

Auch bei der Untersuchung unterschiedlicher Alterskohorten zeigten sich interessante Ergebnisse. Lag bisher aufgrund von einer eingeschränkten Studienlage der Schluss nahe, dass die hüftmorphologische Entwicklung mit Abschluss der Adoleszenz beendet sei, konnten durch diese Studie auch Änderungen der Hüftmorphologie im weiteren Altersverlauf aufgezeigt werden. Entsprechend der Messwerteinteilung nach Altersgruppen ergaben sich signifikante Unterschiede, die somit eine sich auch im Alter fortsetzende physiomorphologische Entwicklung des Hüftgelenks vermuten lassen.

Bei der Analyse einer möglichen Geschlechtsspezifität der Hüftmorphologie konnten ebenfalls für einige Parameter signifikante Messwerte erhoben werden. 
Die unterschiedliche Anatomie des knöchernen Beckens von Frauen und Männern ist langjährig bekannt. In der vorliegenden Arbeit konnte zudem nachgewiesen werden, dass auch beim Hüftgelenk Geschlechtsunterschiede bestehen, was möglicherweise durch die unterschiedliche muskuläre Ausprägung erklärt werden kann.

Beim Vergleich mit Referenzwerten anderer Studien in Bezug auf physiologische und pathologische Werte, zeigten sich interessante Ergebnisse. In dem primär asymptomatischen Kollektiv fand sich für einige Parameter eine Kumulation definitionsgemäß publizierter „pathologischer“ Messwerte (z.B. bei der AcetAVa). Zum Teil erscheinen die Abweichungen von den Referenzwerten dadurch bedingt, dass die physiologischen Werte nur auf jüngere Patientengruppen anwendbar sind. Die altersbedingte morphologische Entwicklung erfordert mit hoher Wahrscheinlichkeit eine Anpassung für Patienten im fortgeschrittenen Lebensalter. Die vorliegende Arbeit konnte drei Parameter (AcetAVa, LCE und SW) für das Acetabulum und einen Parameter für das proximale Femur (FNV) identifizieren, bei denen in der aktuellen Literatur das physiologische Referenzintervall den Ergebnissen der vorliegenden Arbeit zur Folge unzureichend beschrieben erscheint. Für diese Parameter wurde daher in der vorliegenden Arbeit ein neues physiologisches Referenzintervall vorgeschlagen.

Diesen Ausführungen zur Folge, sollten die aktuell publizierten und akzeptierten Referenzwerte zur Hüftgelenkmorphologie aufgrund der hier erhobenen Werte überdacht werden. Basierend auf einer höheren Fallzahl und einer genaueren CT-Analytik erscheinen die Untersuchungsergebnisse valider. Wünschenswert wäre daher ein Heranziehen dieser Daten als neue Referenz bei der Diagnostik und Therapieentscheidung von Hüftgelenkserkrankungen. 


\section{$6 \quad$ Literaturverzeichnis}

Ackerman IN, Kemp JL, Crossley KM, Culvenor AG, Hinman RS (2017): Hip and Knee Osteoarthritis Affects Younger People, Too. J Orthop Sports Phys Ther 47, 67-79

Alberton GM, High WA, Morrey BF (2002): Dislocation after revision total hip arthroplasty: an analysis of risk factors and treatment options. J Bone Joint Surg Am $\underline{84}$, 1788-1792

Ali S, Garcia JM (2014): Sarcopenia, cachexia and aging: diagnosis, mechanisms and therapeutic options - a mini-review. Gerontology $\underline{60}$, 294-305

Altman R, Alarcón G, Appelrouth D, Bloch D, Borenstein D, Brandt K, Brown C, Cooke TD, Daniel W, Feldman D et al. (1991): The American College of Rheumatology criteria for the classification and reporting of osteoarthritis of the hip. Arthritis Rheum $\underline{34}, 505-514$

Amstutz HC, Le Duff MJ. (2019): Sex-specific risk factors determine the survivorship of female and male patients after metal-on-metal hip resurfacing. (im Druck 2020)

Amstutz HC, Le Duff MJ, Beaulé PE (2004): Prevention and treatment of dislocation after total hip replacement using large diameter balls. Clin Orthop Relat Res $\underline{429}, 108-116$

Annett M (1999): The theory of an agnosic right shift gene in schizophrenia and autism. Schizophr Res 39, 177-182

Austin AB, Souza RB, Meyer JL, Powers CM (2008): Identification of abnormal hip motion associated with acetabular labral pathology. J Orthop Sports Phys Ther $\underline{38}, 558-565$

Baghdadi T, Bagheri N, Khabiri SS, Kalantar H (2018): The Outcome of Salter Innominate Osteotomy for Developmental Hip Dysplasia before and after 3 Years Old. Arch Bone Jt Surg $\underline{6}, 318-323$

Bansal A, Khatib ON, Zuckerman, JD (2014): Revision total joint arthroplasty: the epidemiology of 63,140 cases in New York State. J Arthroplasty 29, 23-27

Beck M, Kalhor M, Leunig M, Ganz R (2005): Hip morphology influences the pattern of damage to the acetabular cartilage: femoroacetabular impingement as a cause of early osteoarthritis of the hip. J Bone Joint Surg Br $\underline{87,1012-1018}$

Bentham J, Di Cesare M, Stevens GA, Zhou B, Bixby H, Cowan M, Fortunato L, Bennett JE, Danaei G, Hajifathalian K et al. (2016): A century of trends in adult human height. Elife $\underline{5}$ 
Bernau A (1990): Die Tübinger Hüftbeugeschiene zur Behandlung der Hüftdysplasie. Z Orthop Grenzgeb $\underline{128}, 432-435$

Beverland DE, O'Neill CKJ, Rutherford M, Molloy D, Hill JC (2016): Placement of the acetabular component. Bone Joint J $\underline{98}, 37-43$

Bijlsma JWJ, Berenbaum F, Lafeber FPJG (2011): Osteoarthritis: an update with relevance for clinical practice. Lancet $\underline{377}, 2115-2126$

Buller LT, Rosneck J, Monaco FM, Butler R, Smith T, Barsoum WK (2012): Relationship between proximal femoral and acetabular alignment in normal hip joints using 3-dimensional computed tomography. Am J Sports Med $\underline{40}, 367-375$

Charles MN, Bourne RB, Davey JR, Greenwald AS, Morrey BF, Rorabeck CH (2005): Softtissue balancing of the hip: the role of femoral offset restoration. Instr Course Lect $\underline{54}, 131-141$

Cho YC, Lee PY, Lee CH, Chen CH, Lin YM (2018): Three-dimensional CT Improves the Reproducibility of Stability Evaluation for Intertrochanteric Fractures. Orthop Surg 10, 212 217

Clohisy JC, Carlisle JC, Beaulé PE, Kim YJ, Trousdale RT, Sierra RJ, Leunig M, Schoenecker PL, Millis MB (2008): A systematic approach to the plain radiographic evaluation of the young adult hip. J Bone Joint Surg Am $\underline{4}, 47-66$

Confalonieri N, Manzotti A, Montironi F, Pullen C (2008): Leg length discrepancy, dislocation rate, and offset in total hip replacement using a short modular stem: navigation vs conventional freehand. Orthopedics $\underline{31,10}$

Coventry MB, Beckenbaugh RD, Nolan DR, Ilstrup DM (1974): 2,012 total hip arthroplasties. A study of postoperative course and early complications. J Bone Joint Surg Am $\underline{56}, 273-284$

Cooke WR, Gill HS, Murray DW, Ostlere SJ (2013): Discrete mineralisation of the acetabular labrum. A novel marker of femoroacetabular impingement? Br J Radiol 86, 2012-2182

Croft P, Cooper C, Wickham C, Coggon D (1990): Defining osteoarthritis of the hip for epidemiologic studies. Am J Epidemiol 132, 514-522.

Cruz-Jentoft AJ, Baeyens JP, Bauer JM, Boirie Y, Cederholm T, Landi F, Martin FC, Michel JP, Rolland Y, Schneider SM et al. (2010): Sarcopenia: European consensus on definition and diagnosis: Report of the European Working Group on Sarcopenia in Older People. Age Ageing $\underline{39}, 412-423$ 
Dinser R: Arthrose. In: Dietel M, Suttorp N, Zeitz M (Hrsg.): Harrisons Innere Medizin. Band 3; 18. Auflage; ABW-Verlag, Berlin 2013, 3058-3066

Drenckhahn D, Waschke J: Bewegungsapparat. In: Drenckhahn D, Waschke J (Hrsg.): Taschenbuch Anatomie. 2.Auflage; Urban und Fischer/Elsevier, München 2014, 69-79

Eberhardt O, Wirth T, Fernandez FF (2015): Arthroscopic Anatomy of the Dislocated Hip in Infants and Obstacles Preventing Reduction Arthroscopy. 31, 1052-1059

Eggli S, Pisan M, Müller ME (1998): The value of preoperative planning for total hip arthroplasty. J Bone Joint Surg Br $\underline{80}, 382-390$

Ellison P (2012): Mathematical formulae to calculate the theoretical range of motion of prosthetic hip implants with non-circular neck geometry. Proc Inst Mech Eng H 226, 804-814 Fettweis E (1968): Sitz-Hock-Stellungsgips bei Hüftgelenksdysplasien. Arch Orthop Unfallchir $\underline{63}, 38-51$

Flowers K (1975): Handedness and controlled movement. Br J Psychol 66, 39-52

Gaus W, Muche R: Prinzip des statistischen Test; Chi-Quadrat-Test und andere Test für qualitative Zielgrößen. In: Gaus W, Muche R (Hrsg.): Medizinische Statistik. 2. Auflage; Schattauer, Stuttgart 2014, 264-295

Gerscovich EO (1997): A radiologist's guide to the imaging in the diagnosis and treatment of developmental dysplasia of the hip. I. General considerations, physical examination as applied to real-time sonography and radiography. Skeletal Radiol 26, 386-397

Graf R (1984): Fundamentals of sonographic diagnosis of infant hip dysplasia. J Pediatr Orthop $\underline{4}, 735-740$

Harrasser N, von Eisenhart-Rothe R, Gollwitzer H, Toepfer A, Salzmann M, Berger N, Knebel C, Margraf J, Banke I, Schauwecker J: Untere Extremität. In: Harrasser N, von Eisenhart-Rothe R, Biberthaler P (Hrsg.): Facharztwissen Orthopädie Unfallchirurgie. 1. Auflage; Springer, Berlin, 2016, 369-432

Harris WH (1969): Traumatic arthritis of the hip after dislocation and acetabular fractures: treatment by mold arthroplasty. An end-result study using a new method of result evaluation. J Bone Joint Surg Am. 51, 737-755 
Hartel MJ, Petersik A, Schmidt A, Kendoff D, Nüchtern J, Rueger JM, Lehmann W, Grossterlinden LG (2016): Determination of Femoral Neck Angle and Torsion Angle Utilizing a Novel Three-Dimensional Modeling and Analytical Technology Based on CT Datasets. PLoS One $\underline{2}, 11$

Hartofilakidis G, Bardakos NV, Babis GC, Georgiades G. (2011): An examination of the association between different morphotypes of femoroacetabular impingement in asymptomatic subjects and the development of osteoarthritis of the hip. J Bone Joint Surg Br 93, 580-586

Hasler C, Hefti F: Becken, Hüfte und Oberschenkel. In: Hefti F (Hrsg): Kinderorthopädie in der Praxis. 3. Auflage; Springer, Berlin 2015, 199-277

Hatem MA, da Cunha LAM, Abdo JCM, Martin HD (2017): Parameters for assessment of the inferior acetabulum morphology in 300 adult hips. J Hip Preserv Surg 4, 97-105

Hellstern G: Physiologische Entwicklung und Vorsorgeuntersuchungen. In: Bald M, Biberthaler P, Blattmann C, Bosse HM, Engelmann G, Fitzke G, Freisinger P, Hellstern G, Hempel M, Pape-Feußner N et al. (Hrsg.): Kurzlehrbuch Pädiatrie. 1. Auflage; Thieme, Stuttgart 2012, 43-65

Hetsroni I, Dela TK, Duke G, Lyman S, Kelly BT (2013): Sex differences of hip morphology in young adults with hip pain and labral tears. Arthroscopy 29, 54-63

Hisatome T, Doi H (2011): Theoretically optimum position of the prosthesis in total hip arthroplasty to fulfill the severe range of motion criteria due to neck impingement. J Orthop Sci $\underline{16}, 229-237$

Ji HM, Won SH, Han J, Won YY (2017): Does femoral offset recover and affect the functional outcome of patients with displaced femoral neck fracture following hemiarthroplasty? Injury $\underline{48}, 1170-1174$

Jiang N, Peng L, Al-Qwbani M, Xie GP, Yang QM, Chai Y, Zhang Q, Yu B (2015): Femoral version, neck-shaft angle, and acetabular anteversion in Chinese Han population: a retrospective analysis of 466 healthy adults. Medicine (Baltimore). 94, 21

Kellgren JH, Lawrence JS (1952): Rheumatism in miners. II. X-ray study. Br J Ind Med $\underline{\text {, } 197-}$ 207

Kellgren JH, Lawrence JS (1957): Radiological assessment of osteo-arthrosis. Ann Rheum Dis $\underline{16}, 494-502$ 
Knight JL, Atwater RD (1992): Preoperative planning for total hip arthroplasty. Quantitating its utility and precision. J Arthroplasty $\underline{7}, 403-409$

Kopec JA, Cibere J, Li LC, Zhang C, Barber M, Qian H, Wong H, Steiniger G, Prlich H, Simatovic J (2017): Relationship between physical activity and hip pain in persons with and without cam or pincer morphology. A population-based case-control study. Osteoarthritis Cartilage 25, 1055-1061

Kutzner KP, Pfeil J, Kovacevic MP (2017): Preoperative digital planning versus postoperative outcomes in total hip arthroplasty using a calcar-guided short stem: frequent valgization can be avoided. Eur J Orthop Surg Traumatol 27, 643-651

Lee CB, Kim YJ (2012): Imaging hip dysplasia in the skeletally mature. Orthop Clin North Am $\underline{43}, 329-342$

Lerch TD, Todorski IAS, Steppacher SD, Schmaranzer F, Werlen SF, Siebenrock KA, Tannast M (2018): Prevalence of Femoral and Acetabular Version Abnormalities in Patients With Symptomatic Hip Disease: A Controlled Study of 538 Hips. Am J Sports Med 46, 122-134

Leunig M, Ganz R (2005): Femoroacetabuläres Impingement. Häufige Ursache von zur Arthrose führenden Hüftbeschwerden. Unfallchirurg $\underline{108}, 9-10$

Lewinnek GE, Lewis JL, Tarr R, Compere CL, Zimmerman JR (1978): Dislocations after total hip-replacement arthroplasties. J Bone Joint Surg Am $\underline{60}, 217-220$

Li KC, Higgs J, Aisen AM, Buckwalter KA, Martel W, McCune WJ (1988): MRI in osteoarthritis of the hip: gradations of severity. Magn Reson Imaging $\underline{6}, 229-236$

Litwic A, Edwards MH, Dennison EM, Cooper C (2013): Epidemiology and burden of osteoarthritis. Br Med Bull 105, 185-199

Maheshwari AV, Zlowodzki MP, Siram G, Jain AK (2010): Femoral neck anteversion, acetabular anteversion and combined anteversion in the normal Indian adult population: A computed tomographic study. Indian J Orthop 44, 277-282

Mahmood SS, Mukka SS, Crnalic S, Wretenberg P, Sayed-Noor AS (2016): Association between changes in global femoral offset after total hip arthroplasty and function, quality of life, and abductor muscle strength. A prospective cohort study of 222 patients. Acta Orthop $\underline{87}$, $36-41$ 
Mahoney FI, Barthel DW (1965): Functional evaluation: The Barthel index. Md State Med J $\underline{14}, 61-65$

Mainard D, Barbier O, Knafo Y, Belleville R, Mainard-Simard L, Gross JB (2017): Accuracy and reproducibility of preoperative three-dimensional planning for total hip arthroplasty using biplanar low-dose radiographs: A pilot study. Orthop Traumatol Surg Res 103, 531-536

Mayes S, Ferris AR, Smith P, Garnham A, Cook J (2017): Bony morphology of the hip in professional ballet dancers compared to athletes. Eur Radiol 27, 3042-3049

Mihalko WM, Kammerzell S, Saleh KJ (2009): Acetabular orientation with different pelvic registration landmarks. Orthopedics $\underline{32}, 11-13$

Miyasaka D, Sakai Y, Ibuchi S, Suzuki H, Imai N, Endo N (2017): Sex- and age-specific differences in femoral head coverage and acetabular morphology among healthy subjectsderivation of normal ranges and thresholds for abnormality. Skeletal Radiol $\underline{46}, 523-531$

Müller M, Crucius D, Perka C, Tohtz S (2011): The association between the sagittal femoral stem alignment and the resulting femoral head centre in total hip arthroplasty. Int Orthop $\underline{35}$, $981-987$

Murphy NJ, Eyles JP, Hunter DJ (2016): Hip Osteoarthritis: Etiopathogenesis and Implications for Management. Adv Ther $\underline{33}$, 1921-1946

Nardo L, Parimi N, Liu F, Lee S, Jungmann PM, Nevitt MC, Link TM, Lane NE (2015): Femoroacetabular Impingement. Prevalent and Often Asymptomatic in Older Men: The Osteoporotic Fractures in Men Study. Clin Orthop Relat Res 4굴, 2578-2586

Niethard FU, Pfeil J, Biberthaler P: Hüftgelenk und Oberschenkel. In: Niethard FU, Pfeil J, Biberthaler P (Hrsg.): Orthopädie und Unfallchirurgie. 7., überarb. Auflage; Thieme (Duale Reihe), Stuttgart 2014, 545-548

Nishii T, Sugano N, Miki H, Koyama T, Takao M, Yoshikawa H, (2004): Influence of component positions on dislocation: computed tomographic evaluations in a consecutive series of total hip arthroplasty. J Arthroplasty $\underline{19}, 162-166$

Nötzli HP, Wyss TF, Stoecklin CH, Schmid MR, Treiber K, Hodler J (2002): The contour of the femoral head-neck junction as a predictor for the risk of anterior impingement. J Bone Joint Surg Br $\underline{84}, 556-560$ 
Pagnano W, Hanssen AD, Lewallen DG, Shaughnessy WJ (1996): The effect of superior placement of the acetabular component on the rate of loosening after total hip arthroplasty. $\mathrm{J}$ Bone Joint Surg Am $\underline{78}, 1004-1014$

Patel A, Pavlou G, Mújica-Mota RE, Toms AD (2015): The epidemiology of revision total knee and hip arthroplasty in England and Wales: a comparative analysis with projections for the United States. A study using the National Joint Registry dataset. Bone Joint J $\underline{97}$, 1076-1081

Pavlik A (1957): Die funktionelle Behandlungsmethode mittels Riemenbügel als Prinzip der konservativen Therapie bei angeborenen Huftgelenksverrenkungen der Säuglinge. Z Orthop Grenzgeb $\underline{89}, 341-352$

Preininger B, Schmorl K, von Roth P, Winkler T, Matziolis G, Perka C, Tohtz S (2012): Femoral Offset (3D) in Patients without Osteoarthritis - Index Values from 200 Hip Joints. Open Orthop J $\underline{6}, 578-581$

Renkawitz T: Erkrankungen und Verletzungen von Hüftgelenk und Oberschenkel. In: Grifka J, Kuster M (Hrsg.): Orthopädie und Unfallchirurgie. Für Praxis, Klinik und Facharztprüfung. 1. Auflage; Springer, Berlin 2011, 712-756

Rosenberg IH (1997): Sarcopenia: origins and clinical relevance. J Nutr 127, 990-991

Roubenoff R, Hughes VA (2000): Sarcopenia: current concepts. J Gerontol A Biol Sci Med Sci $\underline{55}, 716-724$

Saberi Hosnijeh F, Kavousi M, Boer CG, Uitterlinden AG, Hofman A, Reijman M, Oei EHG, Bierma-Zeinstra SM, van Meurs JBJ (2018): Development of a prediction model for future risk of radiographic hip osteoarthritis. Osteoarthritis Cartilage 26, 540-546

Sankar WN, Neubuerger CO, Moseley CF (2009): Femoral anteversion in developmental dysplasia of the hip. J Pediatr Orthop $\underline{29}, 885-888$

Sariali E, Mouttet A, Pasquier G, Durante E (2009): Three-dimensional hip anatomy in osteoarthritis. Analysis of the femoral offset. J Arthroplasty 24, 990-997

Sharp IK (1961): Acetabular dysplasia. the acetabular angle. J Bone Joint Surg 43, 268-272

Shi YY, Liu TJ, Zhao Q, Zhang LJ, Ji SJ, Wang EB (2010): The normal centre-edge angle of Wiberg in the Chinese population. A population-based cross-sectional study. J Bone Joint Surg Br 92, 1144-1147 
Stem ES, O'Connor MI, Kransdorf MJ, Crook J (2006): Computed tomography analysis of acetabular anteversion and abduction. Skeletal Radiol 35, 385-389

Suetta C, Haddock B, Alcazar J, Noerst T, Hansen OM, Ludvig H, Kamper RS, Schnohr P, Presctt E, Andersen LL et al. (2019): The Copenhagen Sarcopenia Study: lean mass, strength, power, and physical function in a Danish cohort aged 20-93 years. J Cachexia Sarcopenia Muscle. $\underline{10}, 6$

Suzuki D, Nagoya S, Takashima H, Tateda K, Yamashita T (2017): Three-dimensional orientation of the acetabulum. Clin Anat $\underline{30}$, S. 753-760

Tannast M, Hanke MS, Zheng G, Steppacher SD, Siebenrock KA (2015): What are the radiographic reference values for acetabular under- and overcoverage? Clin Orthop Relat Res $\underline{473}, 1234-1246$

Thielemann F, Olbrich A, Günther KP: Konservative Behandlung in der Kinderorthopädie. In: Bischoff HP, Heisel J, Locher H (Hrsg.): Praxis der konservativen Orthopädie. 1. Auflage; Thieme, Stuttgart 2007, 652-661

Tönnis D, Heinecke A (1999): Acetabular and femoral anteversion. Relationship with osteoarthritis of the hip. J Bone Joint Surg Am $\underline{81}$, 1747-1770

Tohtz SW, Sassy D, Matziolis G, Preininger B, Perka C, Hasart O (2010): CT evaluation of native acetabular orientation and localization: sex-specific data comparison on 336 hip joints. Technol Health Care $\underline{18,}$ 129-136

van Bosse H, Wedge JH, Babyn P (2015): How are dysplastic hips different? A threedimensional CT study. Clin Orthop Relat Res $\underline{473}, 1712-1723$

Wagner M, Breusch SJ, Ewerbeck V, Aldinger PR, Rudert M, Holzapfel BM, Günther KP, Gotterbarm T, Kirschner P, Halder AM et al.: Operation. In: Claes L, Kirschner P, Perka C, Rudert M (Hrsg.): AE-Manual der Endoprothetik. Hüfte und Hüftrevision; Springer, Berlin $2012,161-324$

Wan Z, Boutary M, Dorr LD (2008): The influence of acetabular component position on wear in total hip arthroplasty. J Arthroplasty $\underline{23}, 51-56$

Wells J, Nepple JJ, Crook K, Ross JR, Bedi A, Schoenecker Perry, Clohisy JC (2017): Femoral Morphology in the Dysplastic Hip: Three-dimensional Characterizations With CT. Clin Orthop Relat Res 475, 1045-1054 
Widmer KH (2007): Containment versus impingement: finding a compromise for cup placement in total hip arthroplasty. Int Orthop $\underline{1}$, S29-33

Widmer, KH (2016): Impingementfreie Bewegung nach Hüft-TEP - wie realisieren? Z Orthop Unfall 154, 392-397

Widmer K-H, Zurfluh B (2004): Compliant positioning of total hip components for optimal range of motion. J Orthop Res $\underline{22}, 815-821$

Yoshimine F (2005): The influence of the oscillation angle and the neck anteversion of the prosthesis on the cup safe-zone that fulfills the criteria for range of motion in total hip replacements. The required oscillation angle for an acceptable cup safe-zone. J Biomech $\underline{38}$, 125-132

Zengini E, Finan C, Wilkinson JM (2016): The Genetic Epidemiological Landscape of Hip and Knee Osteoarthritis: Where Are We Now and Where Are We Going? J Rheumatol 43, 260-266 


\section{Danksagung}

An dieser Stelle möchte ich allen herzlich danken, die durch ihre Unterstützung diese Arbeit auf wissenschaftlicher Ebene gefördert haben.

Ein großer Dank gilt der gesamten Forschungsgruppe um meinen initialen Doktorvater Prof. Dr. med. Frank Timo Beil, meinen unmittelbaren Betreuern PD Dr. med. Thelonius Hawellek und PD Dr. med. Jan Hubert sowie den Kooperationspartnern aus der Radiologie, Dr. med. Ali Seif Amir Hosseini und PD Dr. med. Johannes Uhlig. Dabei gilt mein besonderer Dank PD Dr. med. Thelonius Hawellek, der mir stets beratend zur Seite stand, mich zu jeder Zeit bei der Verwirklichung dieser Arbeit unterstützte und umgehend die offizielle Betreuung meiner Dissertation übernahm, als Prof. Dr. med. Beil Göttingen verließ.

Danken möchte ich auch Prof. Dr. med. Arndt Schilling, der mich unvoreingenommen zu einem Gespräch einlud und den Kontakt zu der Forschungsgruppe herstellte sowie Prof. Dr. med. Anna Hell für die Beratung hinsichtlich kinderorthopädischer Aspekte. 


\section{Lebenslauf}

Mein Name ist Marc-Pascal Meier und ich wurde am 04.08.1994 in Göttingen als ältestes von zwei Kindern der Eheleute Stephan Meier und Anja Maus-Meier geboren.

Meine schulische Ausbildung erfolgte ab 2001 an der Grundschule Röddenberg und ab 2005 am Tilman-Riemenschneider-Gymnasium. Als besondere Auszeichnung für meine Leistungen in der Oberstufe wurde mir der Walther-Liebehenz-Schülerpreis der Georg-August-Universität Göttingen verliehen. Nach Erlangung der Hochschulreife im Jahr 2013 (Durchschnittsnote: 1,1) begann ich zum Wintersemester 2013/2014 mein Humanmedizinstudium an der Universitätsmedizin Göttingen.

Im August 2017 wurde ich in die Liste der Promovierenden der Universitätsmedizin Göttingen aufgenommen. $\mathrm{Ab}$ diesem Zeitpunkt arbeitete ich an meinem Promotionsvorhaben. Im Oktober 2018 konnte ich Teile meiner Promotionsarbeit auf dem Jahreskongress der Deutschen Gesellschaft für Orthopädie und Unfallchirurgie präsentieren. Die Ergebnisse wurden dabei so positiv bewertet, dass mir der Posterpreis in der Sektion Grundlagenforschung verliehen wurde. Im November 2019 schloss ich erfolgreich mit dem Bestehen des Dritten Abschnitts der Ärztlichen Prüfung mein Humanmedizinstudium ab. Seit Januar 2020 arbeite ich als Assistenzarzt in der Klinik für Unfallchirurgie, Orthopädie und Plastische Chirurgie der Universitätsmedizin Göttingen. Die Anfertigung der vorliegenden Dissertationsschrift vollendete ich im Februar 2020. 